\title{
Dissertation
}

submitted to the

Combined Faculties for the Natural Sciences and for

Mathematics

of the Ruperto-Carola University of Heidelberg, Germany

for the degree of

Doctor of Natural Sciences

Put forward by

Katharina Isensee, M. Sc.

Born in: Leonberg, Germany

Oral examination: 12.12 .2018 

Towards a quantum cascade laser-based implant for the continuous monitoring of glucose

Referees: Prof. Dr. Wolfgang Petrich Prof. Dr. Peter Bachert 



\section{Towards a quantum cascade laser-based implant for the continuous monitoring of glucose}

The continuous surveillance of the body's glucose concentration is pivotal for the prevention of short- and long-term health complications for people with diabetes. In this thesis, mid-infrared absorption spectroscopy is investigated as an alternative to conventional enzyme-based glucose monitors. To this end, a quantum cascade laser-based sensor system is designed and implemented with the goal to serve as an optical port for continuous, real-time spectroscopy in vivo. This transflection sensor shows high sensitivity in vitro with a glucose error of prediction as low as $4 \mathrm{mg} / \mathrm{dL}$ in pure glucose solutions, $10 \mathrm{mg} / \mathrm{dL}$ in the presence of proteins and $21 \mathrm{mg} / \mathrm{dL}$ in the presence of other carbohydrates. The impact of the temperature on the optical signal is investigated both theoretically and experimentally. Even under temperature variations up to $15^{\circ} \mathrm{C}$ a glucose prediction error as low as $18.5 \mathrm{mg} / \mathrm{dL}$ can be achieved. Adding a porous membrane hinders the diffusion of large molecules into the sensor while enabling glucose diffusion on the targeted time scale $(<5$ minutes). Finally, a stable glucose permeation and concentration prediction over more than 1 month demonstrates the potential of a quantum cascade laser-based transflection technology for application in a long-term implant for continuous glucose sensing.

\section{$\mathrm{Zu}$ einem Quantenkaskadenlaser-basierten Implantat zur kontinuier- lichen Glukosemessung}

Die kontinuierliche Überwachung der Glukosekonzentration des Körpers spielt eine zentrale Rolle in der Prävention von gesundheitlichen Kurz- und Langzeitschäden für Menschen mit Diabetes. In dieser Arbeit wird die Absorptionsspektroskopie im mittleren Infrarot als Alternative zur herkömmlichen Enzym-basierten Glukosemessung untersucht. Zu diesem Zweck wird ein Quantenkaskadenlaserbasiertes Sensorsystem entwickelt und mit dem Ziel implementiert, als optischer Port für die kontinuierliche Echtzeitspektroskopie in vivo zu dienen. Dieser Transflexionssensor zeigt eine hohe Empfindlichkeit in vitro mit einem Vorhersagefehler der Glukosekonzentration von nur $4 \mathrm{mg} / \mathrm{dL}$ in reinen Glukoselösungen, $10 \mathrm{mg} / \mathrm{dL}$ in Gegenwart von Proteinen und $21 \mathrm{mg} / \mathrm{dL}$ in Gegenwart verschiedener Kohlenhydrate. Der Einfluss der Temperatur auf das optische Signal wird sowohl theoretisch als auch experimentell untersucht. Selbst bei Temperaturschwankungen bis zu $15{ }^{\circ} \mathrm{C}$ kann ein Vorhersagefehler der Glukosekonzentration von nur $18.5 \mathrm{mg} / \mathrm{dL}$ erreicht werden. Die Verwendung einer zusätzlichen porösen Membran verhindert die Diffusion von großen Molekülen in den Sensor und ermöglicht gleichzeitig einen ausreichend schnellen ( $<5$ Minuten) Glukoseaustausch. Schließlich zeigt eine über mehr als 1 Monat stabile Glukosepermeation und Konzentrationsvorhersage das Potenzial einer Quantenkaskadenlaserbasierten Transflexionstechnologie für die Anwendung in einem Langzeitimplantat zur kontinuierliche Glukosemessung. 



\section{Contents}

1. Introduction and medical background 9

1.1. Diabetes Mellitus . . . . . . . . . . . . . . . . . . . . 10

1.2. State of the art in mid-infrared based glucose monitoring . . . . . . . 12

2. Materials and methods $\quad 15$

2.1. Spectroscopy . . . . . . . . . . . . . . . . . 16

2.1.1. Vibrational spectroscopy . . . . . . . . . . . . . . 16

2.1.2. Experimental setup . . . . . . . . . . . . . . . . 17

2.1.3. Transflectance . . . . . . . . . . . . . . . 22

2.1.4. Data analysis . . . . . . . . . . . . . . . 23

2.1.5. Optimum layer thickness . . . . . . . . . . . . . . . 33

2.1.6. Data processing . . . . . . . . . . . . . . . 36

2.1.7. Measurements . . . . . . . . . . . . . . . . 39

2.2. Diffusion . . . . . . . . . . . . . . . . . . . . . . . . . 48

2.2.1. Theory of diffusion . . . . . . . . . . . . . . . . 48

2.2.2. Simulation . . . . . . . . . . . . . . . . 49

2.2.3. Measurement parameters . . . . . . . . . . . . . 50

2.3. Long-term measurements . . . . . . . . . . . . . . . . . . . 53

3. Experiments and results $\mathbf{5 5}$

3.1. Spectroscopic quantification of glucose . . . . . . . . . . . . . 56

3.1.1. Measurements of pure glucose solutions . . . . . . . . . . . 56

3.1.2. Environmental impacts . . . . . . . . . . . . . . . . 59

3.2. Glucose diffusion properties . . . . . . . . . . . . . . . . . 77

3.3. Long-term stability . . . . . . . . . . . . . . . . . . . 80

4. Discussion 83

$\begin{array}{ll}\text { A. Appendix } & 107\end{array}$

A.1. Investigation of the impact of temperature dependence of the individual components on the overall measured transflectance . . . . . . . 107

A.2. Simulated absorbance spectra of hypothetical absorber . . . . . . . 108

A.3. Temperature progress curves . . . . . . . . . . . . . . . . 111

$\begin{array}{ll}\text { B. Erklärung } & 117\end{array}$ 



\section{Introduction and medical background}




\subsection{Diabetes Mellitus}

Diabetes mellitus is a metabolic disorder caused by a disfunction in the production and/or effectiveness of insulin in the body [1]. Worldwide 422 million people suffer from diabetes. Since 1980 the number of people with diabetes has quadrupled and is predicted to increase in the near future caused by the increase in standard of living in the so far underdeveloped countries [1]. The disease has a large economic burden on the world. Currently the direct costs of diabetes are more than US $\$ 827$ billion per year [2,3] and it is estimated that they have more than tripled between 2003 and 2013 [4]. In 20121.5 million people died from diabetes [1]. Diabetes is further a cause of "blindness, kidney failure, lower limp amputation and other long-term consequences that impact significantly on quality of life" [1].

Diabetes is related to the pancreas. This organ is very important for the glucose control and metabolism as well as for the digestive system. It contains 3 million 'pancreatic islets' or 'islets of Langerhans' (clusters of cells that are connected to each other) that itself consist of different cell types. Among them are hormone-producing types that are involved in the glucose metabolism: the $\alpha$ cells producing glucagon, the $\beta$ cells producing insulin and amylin, the $\delta$ cells producing somatostatin and the $\gamma$ cells producing pancreatic polypeptide [5]. The cells react to a hormone feedback system as follows: when the blood glucose level is too high the $\beta$ cells produce insulin which triggers the cells in the body to intake glucose. When the glucose level is too low the $\alpha$ cells secret glucagon which triggers the liver cells to emit glucose into the blood stream. The hormone produced by the $\delta$ cells controls the $\alpha$ and $\beta$ cells. In people with diabetes this feedback system is challenged and the disease is distinguished between the following types according to the trigger and course:

- Type 1 diabetes: It is caused by a loss of $\beta$ cells either by an autoimmune attack or by idiopathical causes (unknown cause). As a result, the body does not produce enough insulin to control the glucose metabolism anymore. The insulin has to be injected several times a day to keep the patient alive.

- Type 2 diabetes: The efficiency of insulin on the cells decreases from time to time, the body first compensates by producing more insulin. On the long run this often becomes insufficient and the patient has to add more insulin to its body. Type 2 diabetes is believed to be caused by an interplay of genetic and metabolic factors, such as obesity, lack of physical activity, poor diet or stress. It can in some cases be cured by a change in these lifestyle factors. About $90 \%$ of the diabetes cases are type 2 and $10 \%$ are type 1 diabetes.

- Gestational diabetes: A disorder of the glucose tolerance that appears during the pregnancy caused by the change in metabolism during pregnancy. In most cases it disappears after delivery.

- Other specific types: Other types of insulin insufficiencies or lack of insulin 
producing cells caused by genetic defects, diseases of the pancreas, inflammatory of the pancreas, pancreatic cancer, and others.

Diabetes is usually diagnosed by a repeatedly elevated blood glucose level in the fasting state of a patient or by a oral glucose tolerance test. Here the patient has to digest $75 \mathrm{~g}$ glucose, if the blood glucose level is larger than $200 \mathrm{mg} / \mathrm{dL}$ after two hours the test is positive. The degree of glycation of the protein hemoglobin in the red blood cells, can also be used for the diagnoses. This so called HBA $\mathrm{Hc}_{1 \mathrm{c}}$ (the glycated hemoglobin) is a rating for the average glucose level in the patients plasma over the last 3 months (as the lifetime of red blood cells is about 4 months). The typical blood glucose level in a healthy person lays between $79 \mathrm{mg} / \mathrm{dL}$ to $110 \mathrm{mg} / \mathrm{dL}$ [6]. Patients with (type 1) diabetes can undergo phases with the glucose level way too high (hyperglycemia, $>300 \mathrm{mg} / \mathrm{dL}$ ) and the blood glucose level way too low (hypoglycemia, $<40 \mathrm{mg} / \mathrm{dL}$ ). These states can lead to dangerous short-term complications such as a diabetic coma. Frequent and long-lasting hyperglycemia gives rise to long-term complications such as diseases of the retina (diabetic retinopathy), of the peripheral nervous system (diabetic neuropathy) and kidney insufficiency (diabetic nephropathy).

While type 2 diabetes can mostly be treated with drugs and a more healthy lifestyle (only in some cases insulin treatment is needed), the treatment of type 1 diabetes needs a constant monitor and control of the body's glucose metabolism by glucose measurements and insulin insertion. The continuity and diligence of this treatment is very important as it has been shown that time periods when the blood glucose is exceeding the normal range correspond to a high risk for long term complications such as the above mentioned.

The high number of people with diabetes as well as the risk for complications boots a strong medical and economic driving force for an improvement of the treatment.

As it turned out in the last two decades, the most effective treatment in order to reduce complications for people with type 1 diabetes is to imitate the function of the pancreas as well as possible. This means a continuous constant controlling of the body's metabolism [7]. In the mid of 19th century the first ways of treating diabetes were very harsh and incontinuous as the blood glucose level could not be easily detected and thus patients had to keep a very strict diet. With the development of blood glucose meters (so-called "spot-monitoring") the treatment improved significantly. More recently it has been shown that the use of a continuously monitoring approach instead of a spot-monitoring could improve the treatment even more [8]. The continuous observation enables better estimates of the concentration of glucose over therapeutically important time window (e.g. 15 minutes) and a reaction can be induced much faster [8]. The overall future goal is to couple such a continuous monitoring system with an insulin pump such that an artificial pancreas is created. The patient does not have to actively manage the disease anymore as the system will automatically adapt to the glucose level. This would constitute a large improvement in quality of life for the patient as well as a large decrease in economic costs for the treatment of long-term complications. 


\section{Introduction and medical background}

State of the art spot-like glucose meters have an accuracy of about $10 \mathrm{mg} / \mathrm{dL}$ to $15 \mathrm{mg} / \mathrm{dL}$. Continuous glucose monitoring sensors were developed in the last years: The sensor technology is based on the enzymatic digestion of glucose and the electrochemical quantification (see e.g. [9]). They are applied in a minimally invasive manner which means that they are worn by the patient somewhere in the skin while the electronic hardware is outside the body. Their sensitivity (about $10 \mathrm{mg} / \mathrm{dL}$ to $15 \mathrm{mg} / \mathrm{dL}$ at concentrations smaller $100 \mathrm{mg} / \mathrm{dL}$ and about $10 \%$ to $15 \%$ at concentrations larger than $100 \mathrm{mg} / \mathrm{dL}$ ) is sufficient for the treatment and they have a lifetime of a few weeks [8].

However, the lifetime is limited due to sensor- and enzyme-degradation caused by the reaction product $\mathrm{H}_{2} \mathrm{O}_{2}$ which leads to a loss in glucose sensitivity [8, 9]. Therefore, this $\mathrm{PhD}$ thesis is looking for alternative methods that are applicable for longer periods of time in the range of several months to years. In particular, the reagent-free detection of glucose by means of mid-infrared absorption spectroscopy represents a promising alternative as its lifetime is not limited by such degradation processes.

\subsection{State of the art in mid-infrared based glucose monitoring}

The quantitative investigation of glucose in liquid samples using mid-infrared (MIR) absorption spectroscopy started about 50 years ago. FTIR spectrometers that use a glow-bar as radiation source were used in combination with liquid transmission cells or attenuated total reflection (ATR) geometries ${ }^{1}$. Caused by the high absorption properties of water in the MIR the transmission cells were limited in their layer thickness to few micrometers and the substance-specific absorption signal was very small compared to the one measured in dried samples (see e.g. [10]).

With the invention of the quantum cascade laser as radiation source in the early 90s and with it the chance to deliver high spectral power density to the application, MIR absorption spectroscopy in liquids became experimentally more feasible as the transmission layer thicknesses could be increased to the range of $10 \mu \mathrm{m}$ to several $100 \mu \mathrm{m}$. Glucose and other carbohydrates show characteristic vibrational energies around wavelengths of $10 \mu \mathrm{m}$. The first group that published the potential of this method for the application of glucose measurement in liquids was the group of Martin et al. [11] in 2005. They showed that using a discrete feedback (DFB) QCL and a transmission flow cell with a layer thickness of $41 \mu \mathrm{m}$ a standard error of the mean of glucose prediction of $24.7 \mathrm{mg} / \mathrm{dL}$ can be achieved. Lambrecht et al. [12] showed in another experiment that a fiber-based transmission sensor could be used for the same purpose, making the system more flexible, and gained a noise equivalent

\footnotetext{
${ }^{1}$ ATR geometries have the drawback that the detection medium is limited to a small layer on the surface in the range of the wavelength, which makes inhomogeneous media as e.g. media with proteins that attach to the surface difficult to investigate.
} 
concentrations (NEC) smaller than $10 \mathrm{mg} / \mathrm{dL}$. Vrančić et al. [13] could increase the sensitivity to NEC values as low as $4 \mathrm{mg} / \mathrm{dL}$ using also a fiber-based sensor setup with a discrete frequency Fabry-Perot QCL and an optical path length of $30 \mu \mathrm{m}$. Vrančić et al. [14] also performed the first in vivo experiments using this fiber-based sensor in an animal-model. With a prediction accuracy of $7 \mathrm{mg} / \mathrm{dL}$ and a sampling duration of $6 \mathrm{~s}$ over a period of several hours, they showed that the method is in principle well suited for in vivo use.

All these laser-based methods have in common that they are based on measurements at one fixed frequency, as back then no broadly tunable QCLs were available. This fact hindered the selectivity for glucose in presence of other substances with absorption peaks in the same spectral region. Later experiments showed that this issue can be overcome with tunable external cavity (EC-) QCLs [15-19] and the method became a well established choice for the quantification of all sorts of substances in liquid environment in vitro [20].

Besides this vast progress in the field of in vitro analytics, Vrančić et al. [14] still remain the only group that investigated this method in vivo and built a basis for continuous glucose monitoring in diabetes patients. They used silver/ silver halide fibers in transmission with a microfabricated gap in the fiber to be filled by the liquid via diffusion processes. As this experiment was the first of its kind, it was done in very specific lab conditions for a proof of principle and thus external impacts were kept as low as possible. Anesthetized rats were used to prevent large physical movement of the sensor. Further no investigation of the spectral and biological long-term response and stability was done as the time span before the explantation didn't extent several hours. While these experiments successfully demonstrated the possibility of QCL-based glucose measurements in vivo some difficulties of the fiberbased approach becaume evident, too. In particular the physical movement of the fiber leads to signal changes that effect the glucose prediction.

When thinking towards a long-term glucose monitoring in vivo sensor such difficulties as well as the biocompatibility of the sensor have to be addressed. Furthermore for longer periods of application, proteins could attach inside the the gap of the fiber leading to a decrease in sensitivity. Due to the fibers geometry it is hardly possible to attach a filtering barrier in terms of e.g. a filtration membrane onto the fiber without drastically increasing the sensor's reaction time [21].

The subject of this thesis is to research a compact, long-term, quantum cascade laser-based measurement principle for the continuous glucose monitoring in liquids, that can potentially be integrated in a fully implantable sensor. The experimental investigation of this principle has to cover the following points:

- quantification of the glucose sensitivity in pure glucose solutions using ECQCLs

- quantification of the glucose selectivity in the presence other substances

- measurement of the response time to a glucose concentration change 
- investigation of the impact of a temperature change

- qualitative measurement of long-term stability

- outline of the potential applicability in vivo

These points will be addressed in this thesis starting with the theoretical and experimental background, followed by the experiments and results and a discussion and outlook with respect to an in vivo applicability of the principles.

Starting from the state of the art fiber-based glucose sensor, an approach that fulfills the above given points is worked out. The main driving force is to overcome the difficulties $[17,21,22]$ that occurred with the fiber based sensor approach. Thus the idea to remove the sensitive fibers and built a transmission cell consisting of two parallel windows with a fluid chamber in between comes into mind. However, as the glucose exchange from the surrounding with the inside of the chamber has to be driven by diffusion - a very slow process when compared to active pumping, the diffusion lengths have to be kept as short as possible. In contrast to a transmission measurement in slab geometry a measurement in a transflection chamber appears to be a better approach. Compared to fiber-based transmission spectroscopy it overcomes the geometry problem as now the incoming and outgoing beams are both on the same side. Also the difficulty of long diffusion paths from the side of the measurement chamber can be overcome by implementing micro-holes inside reflective window for fluid exchange from the flat side of the window. This way a fast diffusive exchange between outside and inside the measurement chamber is conceivable. As the exchange takes place through the flat side of the chamber a filtration membrane can easily be attached. This would prevent proteins from getting into the chamber and increase the biocompatibility. For a better biocompatibility of the materials, diamond and gold-coated silicon windows are chosen to built the chamber as these materials are inert and non-toxic. The investigation of different optical incident angles of the beam shows that a small angle in combination with an antireflective coating on the diamond window has proven to be the best option in terms of signalto-noise ratio and stability of the optical adjustment. 
2. Materials and methods 


\subsection{Spectroscopy}

\subsubsection{Vibrational spectroscopy}

Vibrational spectroscopy is a common tool used to detect molecules by probing their characteristic vibrational energies. In the following a brief overview will be given on the physical processes of a molecule situated in an alternating electromagnetic field.

When a molecule is irradiated by an electromagnetic wave, this situation can be seen - to lowest order - as a local coupling between electric field $E(t)$ and the dipole moment of the molecule $\vec{\mu}$. The overall dipole moment of a molecule can then be described by the sum of permanent dipole moment $\vec{\mu}_{0}$ and the induced dipole moment $\vec{\mu}_{\text {induced }}[23]$

$$
\vec{\mu}=\vec{\mu}_{0}+\vec{\mu}_{\text {induced }}=\vec{\mu}_{0}+\hat{\alpha} \vec{E}(t)
$$

with $\hat{\alpha}$ being the polarizability tensor of the molecule. For simplicity, $\alpha$ is assumed here as scalar projection on the direction of the electromagnetic field vector. Further as large molecules are very complex to describe, we assume a diatomic molecule in the following, in which two atoms are covalently bound to each other at a distance $R$ between the nuclei. If the molecule is excited from its equilibrium distance $R_{0}$ e.g. by an electric field of frequency $\omega, \alpha_{0}$ and $\mu_{0}$ change in first order according to

$$
\begin{gathered}
\mu_{0}(R) \approx \mu_{0}\left(R_{0}\right)+\left.\frac{\partial \mu_{0}}{\partial R}\right|_{R=R_{0}}\left(R-R_{0}\right)+\ldots \\
\alpha(R) \approx \alpha\left(R_{0}\right)+\left.\frac{\partial \alpha}{\partial R}\right|_{R=R_{0}}\left(R-R_{0}\right)+\ldots .
\end{gathered}
$$

In harmonic approximation the excitation $R-R_{0}$ itself can be described as

$$
R(t)-R_{0}=A_{\nu} \cos \left(\omega_{\nu} t\right)
$$

with $A_{\nu}$ being the amplitude and $\omega_{\nu}$ being the frequency of the molecular vibration. The local electric field with amplitude $E_{0}$ and frequency $\omega$ can be described as

$$
E_{\text {local }}(t)=E_{0} \cos (\omega) .
$$

Inserting Eq. 2.3 and 2.4 into Eq. 2.2 and using the trigonometric relation $2 \cos (x) \cos (y)=$ $\cos (x+y)+\cos (x-y)$ the following formula can be derived for the molecular dipolemoment

$$
\begin{aligned}
\mu(t)= & \underbrace{\mu_{0}\left(R_{0}\right)}_{\text {permanent dipole moment }}+\underbrace{\left.\frac{\partial \mu_{0}}{\partial R}\right|_{R=R_{0}} A_{\nu} \cos \left(\omega_{\nu} t\right)}_{\text {change in } \mu}+\underbrace{\alpha\left(R_{0}\right) E_{0} \cos (\omega t)}_{\text {elastic scattering }}+ \\
& \underbrace{\left.\frac{E_{0}}{2} \frac{\partial \alpha}{\partial R}\right|_{R=R_{0}} A_{\nu}[\underbrace{\cos \left(\left(\omega-\omega_{\nu}\right) t\right)}_{\text {Stokes }}+\underbrace{\cos \left(\left(\omega+\omega_{\nu}\right) t\right)}_{\text {anti-Stokes }}]}_{\text {inelastic scattering }} .
\end{aligned}
$$


The individual terms in this result can be associated to the following physical properties [23]:

- The first term is the intrinsic permanent dipole moment of the molecule.

- The second term describes dipole transitions that are based on a coherent excitation of vibrational modes at frequencies $\omega_{\nu}$. The energy of this vibrational modes is typically in the range of about $100 \mathrm{meV}$ which matches the mid infrared radiation region. The term $\left.\frac{\partial \mu_{0}}{\partial R}\right|_{R=R_{0}}$ is different from zero for molecules that change their dipole moment when being deflected from their equilibrium position.

- The third term describes the change in dipole moment by elastic scattering (also known as Rayleigh scattering) of an electromagnetic wave with frequency $\omega$.

- The fourth term describes inelastic scattering (also known as Raman scattering) of an electromagnetic wave with frequency $\omega$. It can be divided into a process where the molecule gains energy (Stokes) and one where the molecule looses energy (anti-Stokes, scattering on a molecule that is in an excited state). In contrast to the second term, this effect is based on a change in polarizability $\alpha$ with excitation from the equilibrium position.

One of the largest challenges for the observation of vibrational spectra in biological environment is the abundance and omnipresence of liquid water as a background signal. This can either be overcome by measuring in dried media or - following the Beer-Lambert law - by measuring only very thin layers of the substance. For aqueous solutions, typical layer thicknesses are in the range of $\propto 10^{-4} \mathrm{~m}$ since the absorption coefficient of liquid water amounts to $600 \mathrm{~cm}^{-1}$ [24].

\subsubsection{Experimental setup}

The experimental setup is shown schematically in Fig. 2.1. It consists of two QCLs that emit MIR radiation in different spectral regions and are operated separately. The laser beams are merged on a beam splitter and thus also split into a sample beam path (blue) and a reference beam path (green). The sample beam is focused by a germanium lens (PCX, Edmund Optics Ltd-, UK, focal length $\mathrm{f}=25 \mathrm{~mm}$ ) onto the transflection opto-fluidic interface (Fig. 2.2), is reflected, collimated again by the same lens and then collected by a pyroelectric detector (LME-352-63, Infratec $\mathrm{GmbH}$, Germany). The reference beam is used for detection of intensity variations in the laser's output and detected directly by another pyroelectric detector. The laser beam is modulated by a chopper wheel in order to use lock-in detection (SR 810, SR 830. Stanford Research Systems, USA) for amplification and detection of the signals. The modulation frequency is chosen to be $421 \mathrm{~Hz}$. The time constant of the lock-in amplification is $300 \mathrm{~ms}$ which corresponds to a wavenumber resolution of 


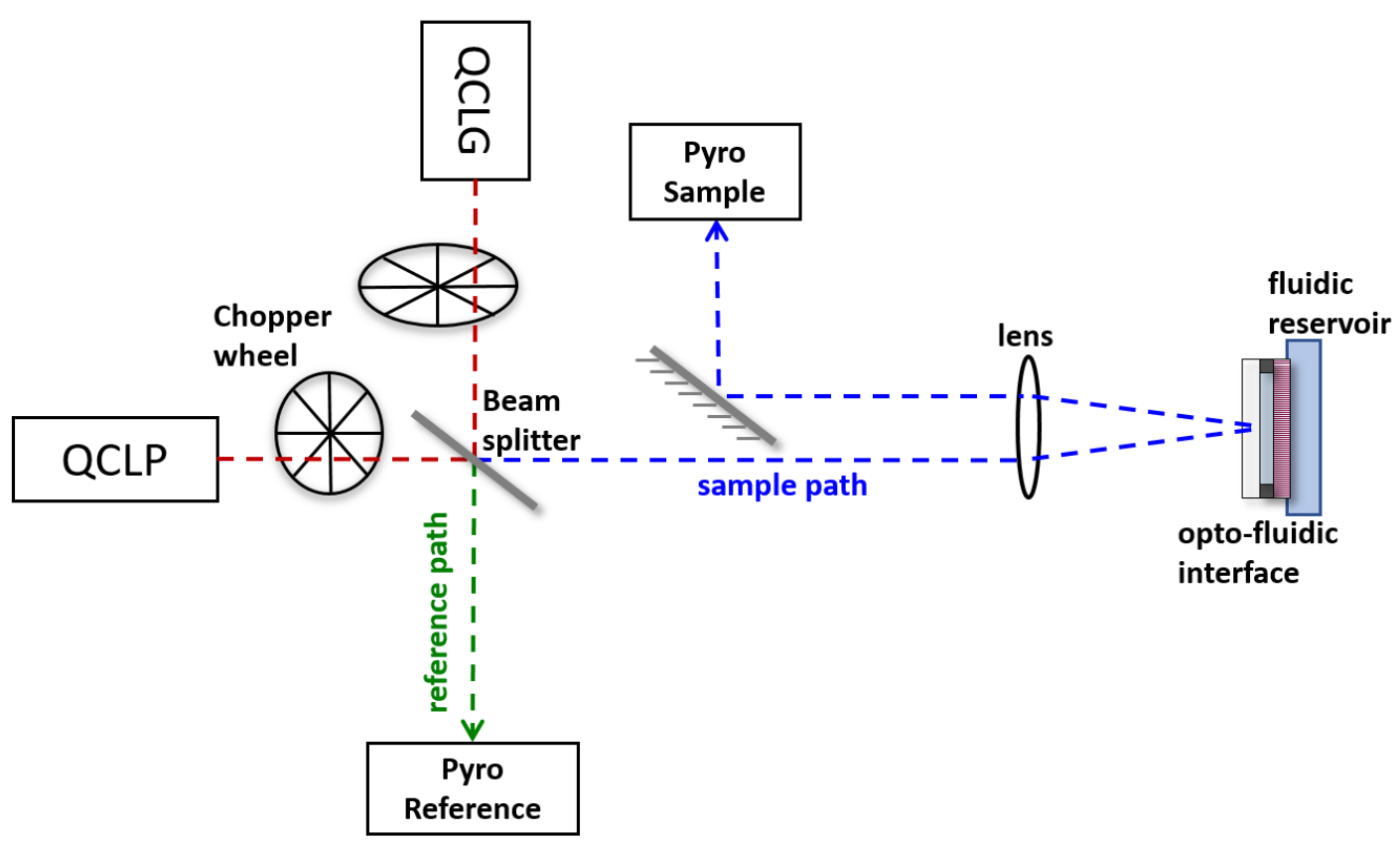

Figure 2.1.: Schematic of the optical path. The laser beams are merged by a beam splitter and split up into a sample and reference beam. The reference beam is detected to correct for intensity fluctuations and the sample beam passes the opto-fluidic interface, gets reflected and detected with a pyroelectric detector. Detection takes place with lock-in detection using a periodically modulated beam. Figure adapted from Isensee et al. [25].

$5 \mathrm{~cm}^{-1}$ when scanning the laser's wavelength over time (see also Sec. 2.1.2 Quantum cascade laser).

\section{Opto-fluidic interface}

The heart of the setup is the reflecting opto-fluidic interface (Figure 2.2). A measurement chamber is formed by a diamond window (CVD diamond, $400 \mu \mathrm{m}$ thick, anti-reflection coated, Diamond Materials GmbH, Germany) and a silicon wafer (200 $\mathrm{mm}$ thick, Silicon Materials, Germany). Both components are held apart by a stainless steel spacer ring with a thickness of 10(2) $\mu \mathrm{m}$. In a transflection measurement the incident beam is shown in from the top, gets transmitted through the diamond window, passes the liquid layer, gets reflected at the surface of the silicon wafer, passes the liquid layer and the diamond window again, before it gets detected. For a higher intensity of the reflected signal, the reflectivity of the silicon wafer is increased by a $150 \mathrm{~nm}$ gold film on its upper surface.

The volume between the window and the silicon wafer can be filled with a liquid from outside. For fluid exchange, the silicon wafer is interspersed with small conical holes with a diameter of about $10 \mu \mathrm{m}$ at the upper and about $45 \mu \mathrm{m}$ on the lower surface aligned in a hexagonal pattern with a hole to hole distance of $50 \mu \mathrm{m}$. A 
sketch and light microscope images are shown in Figure 2.3 and 2.4. The beam width of the focused MIR beam inside the measurement chamber is about $1 \mathrm{~mm}$ such that the area probed by the beam contains about 310 holes on average.

Optionally an additional filtration membrane can be attached below the silicon wafer in order to get an additional filtering effect. On the other side of the silicon wafer a fluidic reservoir is attached. This can be filled with different solutions by a peristaltic pump (HiTec Zang GmbH, Germany) in combination with an automatic valve (Knauer GmbH, Germany).

For the investigation of the temperature dependence and in order to heat the sensor to a particular temperature the opto-fluidic interface is equipped with a PT100 temperature sensor (Heraeus Sensor Technology GmbH, Germany) and a heating tape element (OMEGA Engineering Inc., USA), both coupled by a digital PID controller. The temperature sensor is mounted on the stainless steel housing that holds the layer stack of windows, as close as possible to the measurement chamber itself.

\section{Quantum cascade laser}

Quantum cascade lasers (QCLs) were invented in the end of 19th century and first demonstrated by Faist et al. [26] in 1994. In contrast to 'classical' diode lasers, where the lasing principle is based on the recombination of electron-hole pairs across the material's band gap, QCLs are based on intraband-transitions of electrons within a semiconductor heterogeneous quantum well structure. This structure consists of alternating layers of injector and active regions which constitute a multiple quantum well confinement. When a voltage is applied, a population inversion between an excited state and a ground state within a quantum well is achieved. An electron can propagate from one quantum well to another by tunneling. Thus, one electron can emit a photon at every step - a cascade of photons is produced by a single electron. Depending on the material and thickness of the individual layers the wavelength of the emitted radiation can be designed. So far QCLs for the MIR and terahertz (farIR) region are available. The wavelength selection is done either by temperature tuning the laser's cavity (Fabry-Perot QCLs) or by an external mechanical grating (external cavity QCLs). Nowadays tunable QCLs are used in various applications such as gas-absorption spectroscopy, microscopy and many biomedical applications [27-29].

For the experiments described in this thesis external cavity (EC-) QCLs (Daylight Solutions Inc., USA) emitting in the spectral regions of $900 \mathrm{~cm}^{-1}$ to $1200 \mathrm{~cm}^{-1}$ (QCLG) and $1500 \mathrm{~cm}^{-1}$ to $1750 \mathrm{~cm}^{-1}$ (QCLP) are used. They can be operated in pulsed and continuous wave $(\mathrm{cw})$ mode and have a pointing stability of $<1 \mathrm{mrad}$ and a bandwidth $<1 \mathrm{~cm}^{-1}$. The lasers' output is linearly (horizontally) polarized. A detailed investigation of the long-term stability when constantly scanning the lasers wavelength over the lasers gain profile, showed that despite the overall lower power in pulsed mode, this mode is still up to 3 order of magnitudes more stable than the $\mathrm{cw}$ mode: This can be seen in the acquired absorbance noise with respect to integration time evaluated by an Allan variance formalism (as introduced e.g. by Lambrecht 


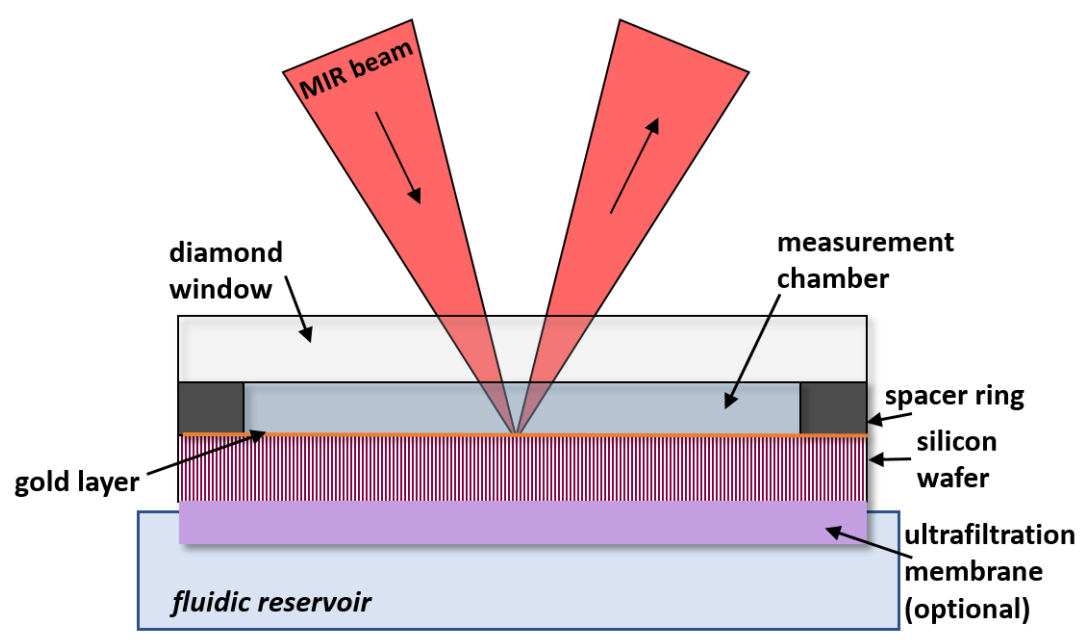

Figure 2.2.: Schematic of the opto-fluidic interface. The interface consists of a layer stack of a diamond window, a stainless steel spacer ring and a holepatterned silicon wafer sitting on top of a fluidic reservoir. The diamond window has an antireflective coating on its surface and the silicon wafer has a $150 \mathrm{~nm}$ gold film on the side pointing towards the measurement chamber. The spacer ring keeps the two windows at a constant thickness $(10(2) \mu \mathrm{m})$ in order to generate a constant measurement chamber in between which can be filled with liquid by means of diffusion through the hole-patterned silicon wafer. Optionally an additional ultrafiltration membrane can be attached at the side pointing towards the fluidic reservoir. The laser beam (indicated in red) is focused onto the reflective gold film below the measurement chamber, thereby it passes the substance of interest, gets reflected, passes the substance of interest again and gets directed towards the detector as shown in Fig. 2.1. Figure adapted from Isensee et al. [25]. 


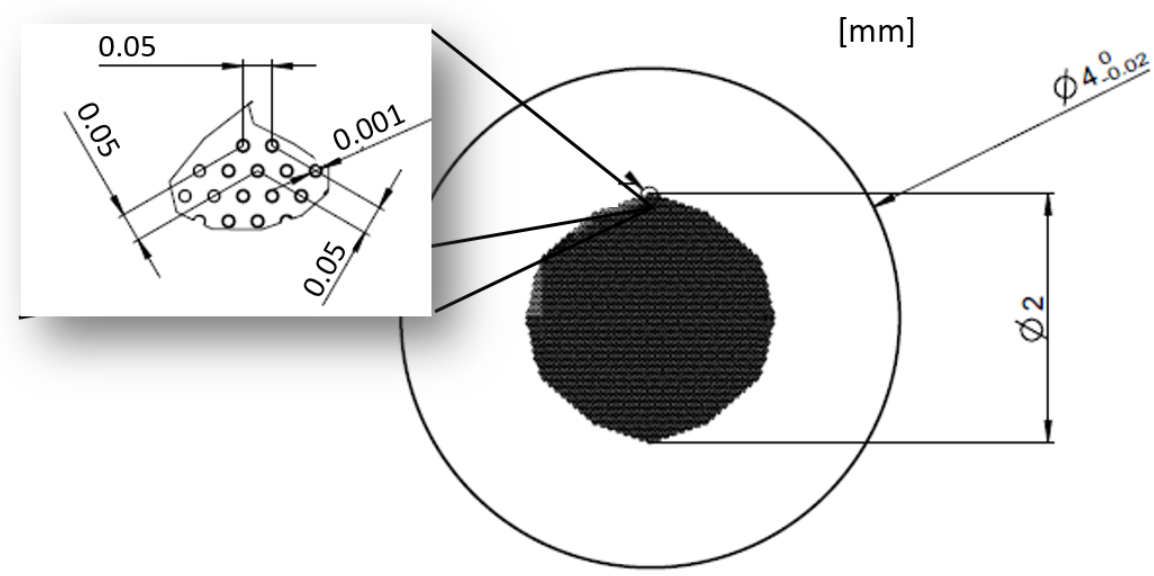

Figure 2.3.: Sketch of the silicon wafer and the hole pattern that has been manufactured at it. Figure adapted from Isensee et al. [25].
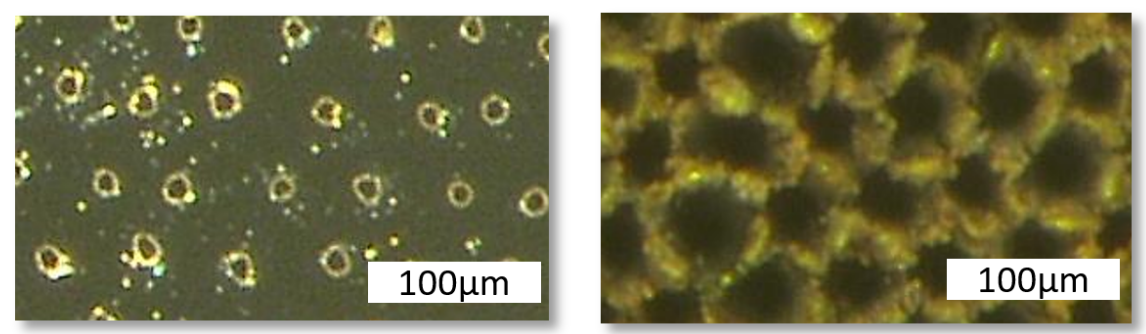

Figure 2.4.: Light microscope images of the upper (left) and lower (right) surface of the micro-structured silicon wafer. Figure adapted from Isensee et al. $[25]$. 


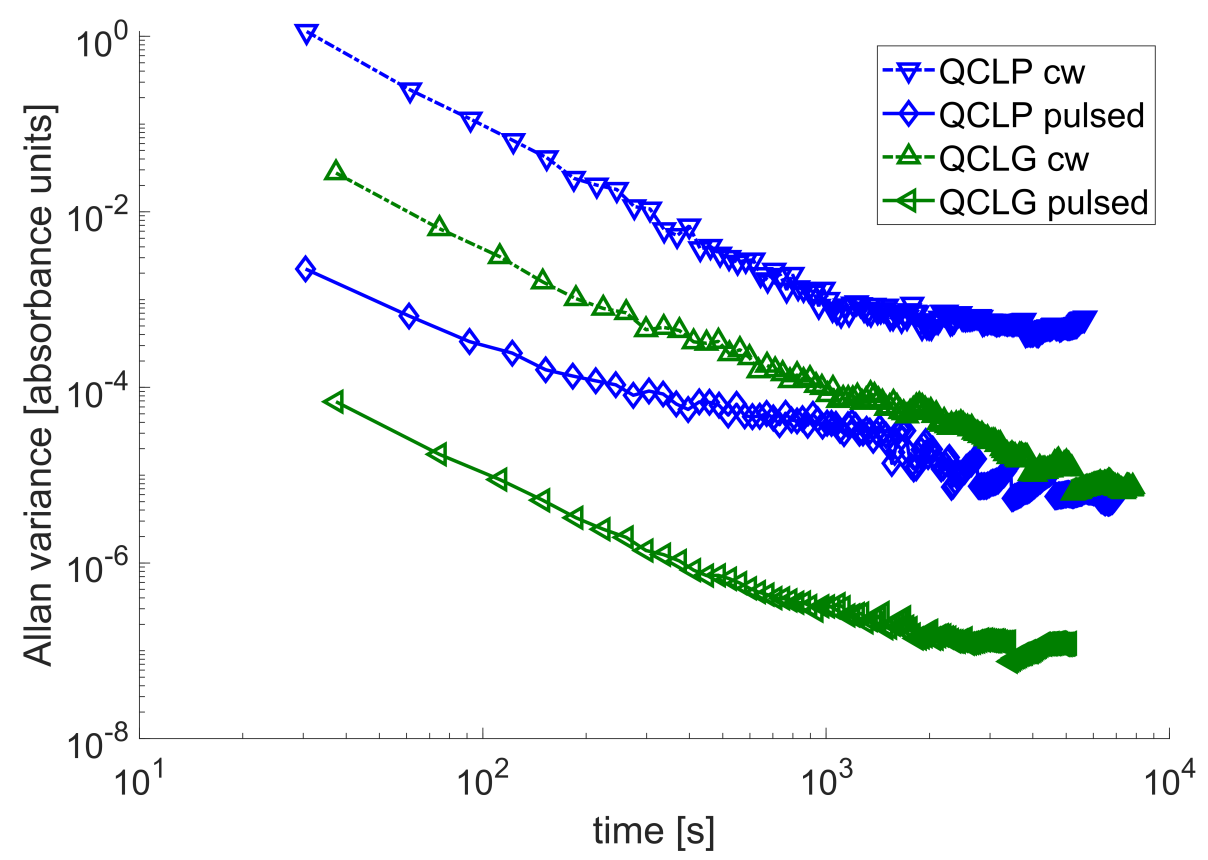

Figure 2.5.: Allan variance for the characterization of the long-term stability of the raw laser output signal with respect to integration time. The Allan variance is calculated according to $[12,22]$ on the decadic absorbance spectra with the very first spectrum taken as reference when calculating the absorbance. The Allan variance values obtained in pulsed mode are up to 3 orders of magnitude smaller than the ones obtained in $\mathrm{cw}$ mode (dashed lines). The spectra are acquired by continuously sweep scanning the lasers wavelength over time and detecting the direct laser output signal.

et al. [12], Vrancî́c [22], see Figure 2.5) . Hence the lasers are operated in pulsed mode for all experiments shown in this thesis (repetition rate $100 \mathrm{kHz}$, pulse width $500 \mathrm{~ns}$, duty cycle $5 \%$ ). The experimental operation is done in a continuous sweep scan mode where the lasers wavenumber is continuously changed while the signal is simultaneously measured by the pyroelectric detectors. The scanning times amount to $17 \mathrm{~s}$ and $14 \mathrm{~s}$ for QCLG and QCLP, respectively. The spectral emission profile is calibrated once prior to all experiments by measurement of an ethanol gas spectrum (QCLG) and a water vapor spectrum (QCLP) and assigning the absorption bands to literature [30, 31]. Figure 2.6 shows the obtained lasers' emission profiles.

\subsubsection{Transflectance}

The transflectance $A$ is calculated according to

$$
A=-\log _{10}\left(I_{\text {substance }} / I_{\text {reference }}\right)
$$




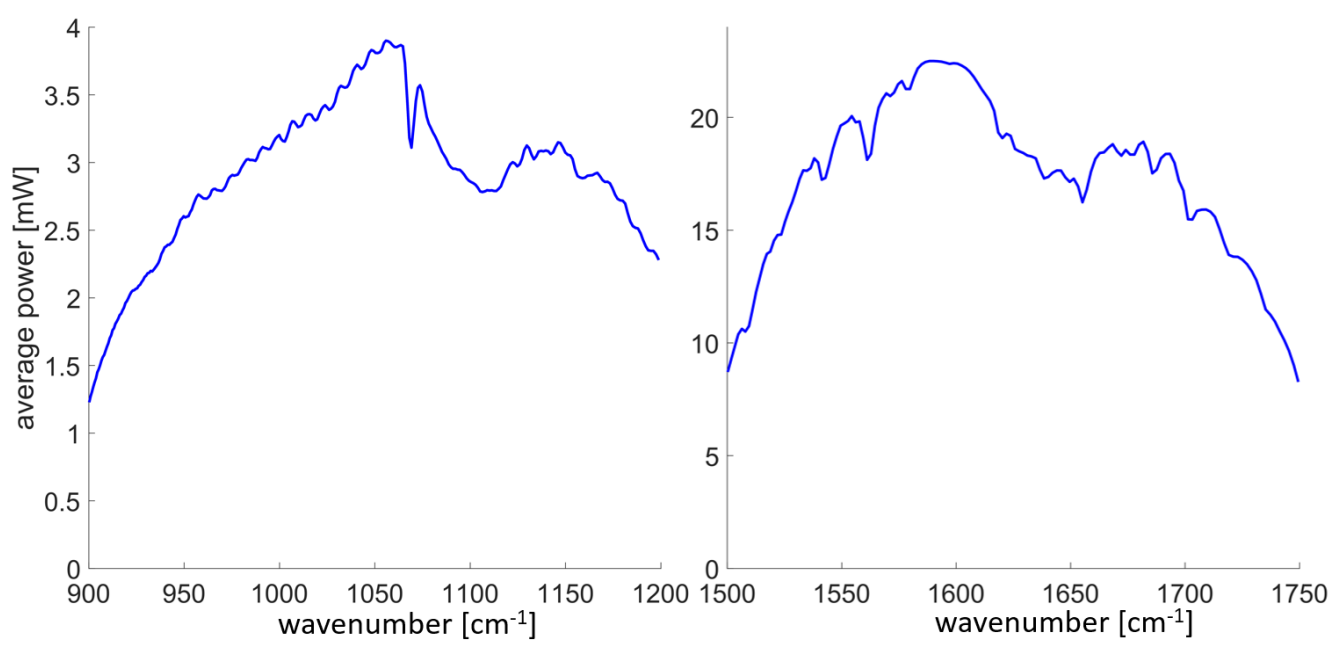

Figure 2.6.: Spectral laser emission profiles of QCLG $\left(900 \mathrm{~cm}^{-1}\right.$ to $\left.1200 \mathrm{~cm}^{-1}\right)$ and QCLP $\left(1500 \mathrm{~cm}^{-1}\right.$ to $\left.1750 \mathrm{~cm}^{-1}\right)$ measured in pulsed operation mode.

with $I_{\text {substance }}$ being the measured transflected signal with the measurement chamber filled with a substance of interest and $I_{\text {reference }}$ being the measured transflected signal with the measurement chamber filled with a reference liquid. As will be shown in the following, the (decadic) absorbance as it is defined in transmission geometry can not be directly compared to the transflectance as the signals $I$ show spectral modifications caused by nonlinear coherent effects. To make this clear, in this thesis the term transflectance is used for data measured in transflection and the term absorbance is used for data measured in transmission.

\subsubsection{Data analysis}

The transflection setup (or opto-fluidic interface) described above contains several parallel thin layers. An incoming electromagnetic wave is split up into a reflected part and a transmitted part at every border. In principle, when an electromagnetic wave gets reflected at multiple boundaries, the incident and reflected waves form a standing wave, which can be theoretically described by the solution of Maxwell's equations (see e.g. [32]). Thus, the overall reflected radiation of a sample with several parallel layers is an interfered signal from contributions of all layers. The reflected signal strongly depends on the optical and geometrical properties of the individual layers and interfaces such as e.g. the complex refractive index and the thickness of each layer.

This phenomenon makes quantitative measurements in transflection somewhat challenging, especially when looking at samples with inhomogeneous sample thickness and inhomogeneous refractive indices (see e.g. [33]). Although long known in principle (see e.g. [32]), several research groups have more recently investigated the potential of MIR transflection measurements and the impact of the above described 


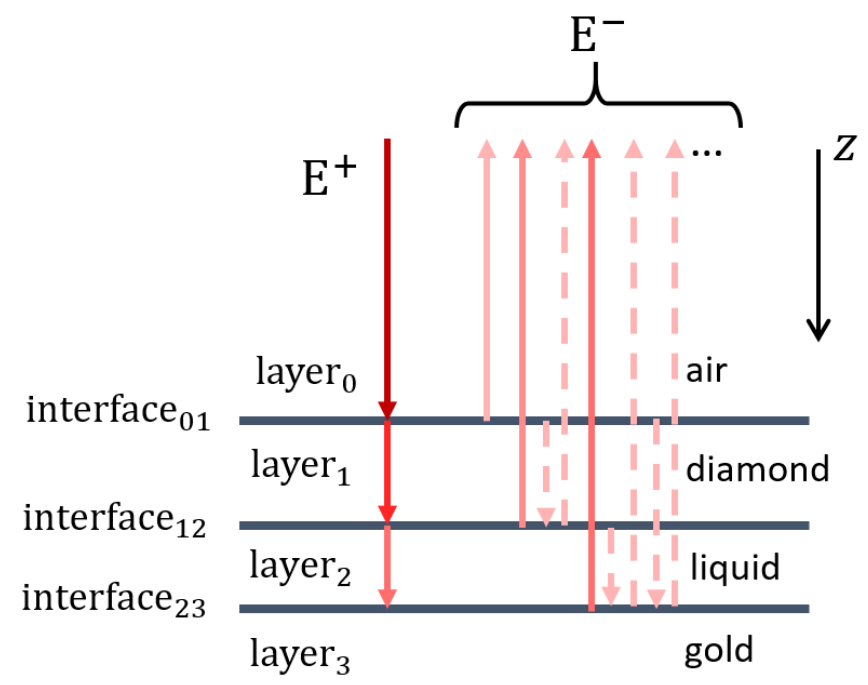

Figure 2.7.: Schematic of the different interfaces in the sensor setup consisting of the layers air $\left(\right.$ layer $\left._{0}\right)$, diamond $\left(\right.$ layer $\left._{1}\right)$, liquid (i.e. water, layer 2 ) and gold (layer ${ }_{3}$ ). The arrows indicate the incoming and reflected outgoing electromagnetic waves (first order reflection as well as higher order reflections (indicated in dashed lines)). The complex amplitude of the electromagnetic field traveling towards the interface is defined as $\mathrm{E}^{+}$ and the one traveling backwards is defined as $\mathrm{E}^{-}$.

phenomenon [33-40]. Brooke et al. [40] for example showed that this effect leads to spectrally nonlinear distortions in the absorbance signal with respect to the layer thickness of the investigated medium [33, 40]. It is important to note, though, that our liquid samples are homogeneous in contrast to the structured tissue in references [33-40].

To understand the reflected infrared signal measured with the sensor and its dependency on different optical and environmental parameters in detail, a basic theoretical model is introduced in the following. This model is used to calculate the detected intensities with respect to the different parameters and also to predict the spectral shape of a typical transflectance spectrum.

\section{Calculation of the signal}

The goal is to gain a theoretical calculation of the overall intensity $I_{\text {reflected }}$ corresponding to the signals measured in the experiments. Due to the high coherence of the laser light and the substructure sizes in the range of the wavelength, interference effects have to be taken into account. A calculation of the electromagnetic fields can be done by, for example, a matrix-formalism such as the one introduced by Azzam and Bashara [41]: A scattering matrix is calculated for a stack of parallel, distinct layers. It is assumed that the optical properties are uniform within each layer and that the optical properties change abruptly at the interface between the individ- 
ual layers. The incoming electromagnetic wave is described as a monochromatic plane wave with its polarization either parallel $(\mathrm{p})$ or perpendicular $(\mathrm{s})$ to the plane of incidence. The total electromagnetic field $\overrightarrow{\mathbf{E}}$ at a position $z$ (the z-direction is perpendicular to the layer interfaces) is:

$$
\overrightarrow{\mathbf{E}}=\left(\begin{array}{l}
\mathrm{E}^{+}(z) \\
\mathrm{E}^{-}(z)
\end{array}\right)
$$

with $\mathrm{E}^{+}(z)$ and $\mathrm{E}^{-}(z)$ being the complex amplitudes of the forward and backward traveling waves (with respect to $z$ ). When an electromagnetic wave $\overrightarrow{\mathbf{E}}$ passes a layer interface the wave on one side (position $z^{\prime}$ ) of the interface $\overrightarrow{\mathbf{E}}\left(z^{\prime}\right)$ can be related to the one on the other side (position $z^{\prime \prime}$ ) by a scattering matrix $\hat{\mathbf{S}}$.

$$
\begin{aligned}
\overrightarrow{\mathbf{E}}\left(z^{\prime}\right) & =\hat{\mathbf{S}} \overrightarrow{\mathbf{E}}\left(z^{\prime \prime}\right) \\
\left(\begin{array}{l}
\mathrm{E}^{+}\left(z^{\prime}\right) \\
\mathrm{E}^{-}\left(z^{\prime}\right)
\end{array}\right) & =\left[\begin{array}{ll}
S_{11} & S_{12} \\
S_{21} & S_{22}
\end{array}\right]\left(\begin{array}{l}
\mathrm{E}^{+}\left(z^{\prime \prime}\right) \\
\mathrm{E}^{-}\left(z^{\prime \prime}\right)
\end{array}\right)
\end{aligned}
$$

with $\hat{\mathbf{S}}$ being characteristic for the structure between $z^{\prime}$ and $z^{\prime \prime} . \hat{\mathbf{S}}$ can thereby consist of a product representing the effect of all layers and interfaces in the order as they are present. The propagation through a layer $a$ can be described by the matrix $\hat{\mathbf{L}}_{a}$ as

$$
\hat{\mathbf{L}}_{a}=\left[\begin{array}{cc}
e^{i \beta_{a}} & 1 \\
1 & e^{-i \beta_{a}}
\end{array}\right]
$$

with $\beta_{a}$ being calculated by

$$
\beta_{a}=\frac{2 \pi d_{a} N_{a}}{\lambda} \cos \left(\phi_{a}\right)=2 \pi d_{a} N_{a} \tilde{\nu} \cos \left(\phi_{a}\right)
$$

with $d_{a}$ being the thickness of the layer a, $N_{a}$ being the complex index of refraction of the layer (Eq. 2.14), $\lambda$ being the wavelength, $\phi_{a}$ being the angle (can also be complex) of the propagation direction compared to perpendicular to the boundary. The impact of an interface can be described by the matrix $\hat{\mathbf{I}}_{a b}$ (between layer a and b) as

$$
\hat{\mathbf{I}}_{a b}=\left[\begin{array}{cc}
1 / t_{a b} & r_{a b} / t_{a b} \\
r_{a b} / t_{a b} & 1 / t_{a b}
\end{array}\right]=1 / t_{a b}\left[\begin{array}{cc}
1 & r_{a b} \\
r_{a b} & 1
\end{array}\right]
$$

with $r_{a b}$ and $t_{a b}$ being the complex reflection and transmission coefficients that can be calculated by the complex refractive index of the adjacent layers, as well as the local angle of incidence, using Fresnel's equation (Equation 2.12, $s$ and $p$ indicating 
the different polarizations).

$$
\begin{aligned}
r_{a b}^{p} & =\frac{N_{b} \cos \left(\phi_{a}\right)-N_{a} \cos \left(\phi_{b}\right)}{N_{b} \cos \left(\phi_{a}\right)+N_{a} \cos \left(\phi_{b}\right)} \\
r_{a b}^{s} & =\frac{N_{a} \cos \left(\phi_{a}\right)-N_{b} \cos \left(\phi_{b}\right)}{N_{a} \cos \left(\phi_{a}\right)+N_{b} \cos \left(\phi_{b}\right)} \\
t_{a b}^{p} & =\frac{2 N_{a} \cos \left(\phi_{a}\right)}{N_{b} \cos \left(\phi_{a}\right)+N_{a} \cos \left(\phi_{b}\right)} \\
t_{a b}^{s} & =\frac{N_{a} \cos \left(\phi_{a}\right)}{N_{a} \cos \left(\phi_{a}\right)+N_{b} \cos \left(\phi_{b}\right)} .
\end{aligned}
$$

The local angles of incidence with respect to the normal of the interface can be calculated by Snell's law as

$$
N_{a} \sin \phi_{a}=N_{b} \sin \phi_{b}
$$

with $\phi_{a}$ being the angle of incidence and $\phi_{b}$ being the refraction angle. The complex refractive index is defined as

$$
N_{a}=n_{a}-i k_{a}
$$

with $n_{a}$ being the real and $k_{a}$ being the imaginary part. The absorption coefficient $\alpha_{a}$ of an absorbing medium/layer $a$ relates to $k_{a}$ via

$$
k_{a}=\frac{\lambda \alpha_{a}}{4 \pi}=\frac{\alpha_{a}}{4 \pi \tilde{\nu}} .
$$

For the here described system (Fig. 2.7) $\hat{\mathbf{S}}$ can be constructed by a product of the interface-matrices and the layer-matrices of the two distinct layers (layer 1 and 2) according to:

$$
\begin{aligned}
\hat{\mathbf{S}}= & {\left[\begin{array}{ll}
S_{11} & S_{12} \\
S_{21} & S_{22}
\end{array}\right]=\hat{\mathbf{I}}_{01} \hat{\mathbf{L}}_{1} \hat{\mathbf{I}}_{12} \hat{\mathbf{L}}_{2} \hat{\mathbf{I}}_{23} } \\
= & \left(\frac{1}{t_{01} t_{12} t_{23}}\right)\left[\begin{array}{cc}
1 & r_{01} \\
r_{01} & 1
\end{array}\right]\left[\begin{array}{cc}
e^{i \beta_{1}} & 1 \\
1 & e^{-i \beta_{1}}
\end{array}\right]\left[\begin{array}{cc}
1 & r_{12} \\
r_{12} & 1
\end{array}\right]\left[\begin{array}{cc}
e^{i \beta_{2}} & 1 \\
1 & e^{-i \beta_{2}}
\end{array}\right]\left[\begin{array}{cc}
1 & r_{23} \\
r_{23} & 1
\end{array}\right] \\
= & \left(\frac{e^{i\left(\beta_{1}+\beta_{2}\right)}}{t_{01} t_{12} t_{23}}\right)\left[\begin{array}{c}
\left(1+r_{01} r_{12} e^{-i 2 \beta_{1}}\right)+\left(r_{12}+r_{01} e^{-i 2 \beta_{1}}\right) r_{23} e^{-i 2 \beta_{2}} \\
\left(r_{01}+r_{12} e^{-i 2 \beta_{1}}\right)+\left(r_{01} r_{12}+e^{-i 2 \beta_{1}}\right) r_{23} e^{-i 2 \beta_{2}} \\
\left(1+r_{01} r_{12} e^{-i 2 \beta_{1}}\right) r_{23}+\left(r_{12}+r_{01} e^{-i 2 \beta_{1}}\right) e^{-i 2 \beta_{2}} \\
\left(r_{01}+r_{12} e^{-i 2 \beta_{1}}\right) r_{23}+\left(r_{01} r_{12}+e^{-i 2 \beta_{1}}\right) e^{-i 2 \beta_{2}}
\end{array}\right] .
\end{aligned}
$$

By the definition of the overall reflection coefficient $\mathrm{R}$ as the quotient of the outgoing to the incoming electromagnetic wave and the fact that there is no ingoing wave on the side of the gold layer the overall reflection coefficient $\mathrm{R}$ can be calculated by

$$
R=\frac{\mathrm{E}^{-}}{\mathrm{E}^{+}}=\frac{S_{21}}{S_{11}}
$$


As we are interested in the intensity $I \propto|\overrightarrow{\mathbf{E}}|^{2}$ the reflected intensity can be calculated by

$$
\begin{aligned}
I_{\text {reflected }} & =I_{\text {incident }}|R|^{2}=I_{\text {incident }} \frac{\left|E^{-}\right|^{2}}{\left|E^{+}\right|^{2}}=I_{\text {incident }} \frac{\left|S_{21}\right|^{2}}{\left|S_{11}\right|^{2}} \\
& =I_{\text {incicent }} \frac{\left|\left(r_{01}+r_{12} e^{-i 2 \beta_{1}}\right)+\left(r_{01} r_{12}+e^{-i 2 \beta_{1}}\right) r_{23} e^{-i 2 \beta_{2}}\right|^{2}}{\left|\left(1+r_{01} r_{12} e^{-i 2 \beta_{1}}\right)+\left(r_{12}+r_{01} e^{-i 2 \beta_{1}}\right) r_{23} e^{-i 2 \beta_{2}}\right|^{2}} .
\end{aligned}
$$

Further details about the theoretical calculation can be found e.g. in [32, 41].

When comparing the simulated signal to the measured one, the spatial expansion of the beam as well as the time-resolution of the lock-in amplifier have to be kept in mind:

- The spatial distribution of the beam leads to a subset of incidence angles to the layer stack, as schematically shown in Figure 2.8. The intensity distribution $g$ is chosen to be a Gaussian distribution with a full width half maximum of $D_{\text {beam }} / 2$. Using the position of the center of the beam on the lens $x_{\text {center }}$ together with the focal length $f$ of the lens, the corresponding incident angle $\phi$ can be calculated for each respective position $x$. For simplification only a two dimensional Gaussian is calculated. Given the small angle of incidence in the experiments, a rotational symmetry may be assumed for the incoming beam such that the integration of the 2D Gaussian over a half sphere provides a good estimate for the 3-dimensional case.

- The time resolution of the lock-in amplifier (integration time $300 \mathrm{~ms}$ ) leads to a spectral resolution of about $5 \mathrm{~cm}^{-1}$ (based on the scanning speed of the QCL sweep scan). To include this into the simulated signal a moving-average filter with a width of $5 \mathrm{~cm}^{-1}$ is applied on $I_{\text {reflected }}$.

After calculating $I_{\text {reflected }}$ at every position $x$ the total signal is derived by summing up all intensities.

\section{Signal simulation}

The above method is implemented in Python and the following input parameters for the different layer matrices are used:

- Air-diamond interface $\hat{\mathbf{I}}_{01}$ : The diamond window used in the experiment is coated with an anti-reflection coating. The reflectivity $\left|r_{01}\right|^{2}$ of this coating is measured with a FTIR microscope (Hyperion 1000, Bruker Optik GmbH, Deutschland) in reflection as shown in Figure 2.9. In the spectral region relevant for the experiment $\left(900 \mathrm{~cm}^{-1}\right.$ to $1200 \mathrm{~cm}^{-1}, 1500 \mathrm{~cm}^{-1}$ to $1750 \mathrm{~cm}^{-1}$ ) a reflectivity $\left|r_{01}\right|^{2}$ smaller than $2 \%$ is observed. The square root of this reflectivity is inserted into the interface matrix $\hat{\mathbf{I}}_{01}$. 


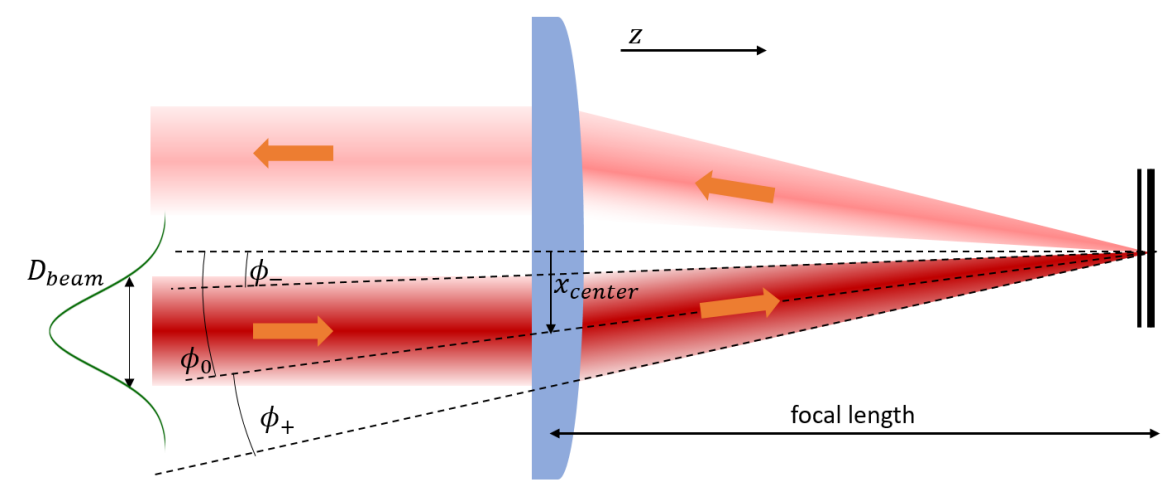

Figure 2.8.: Schematic of the incident beam geometry. The beam with a diameter in the range of a few millimeters is focused on the sensor setup thus leading to a distribution of different angles and different incident intensities.

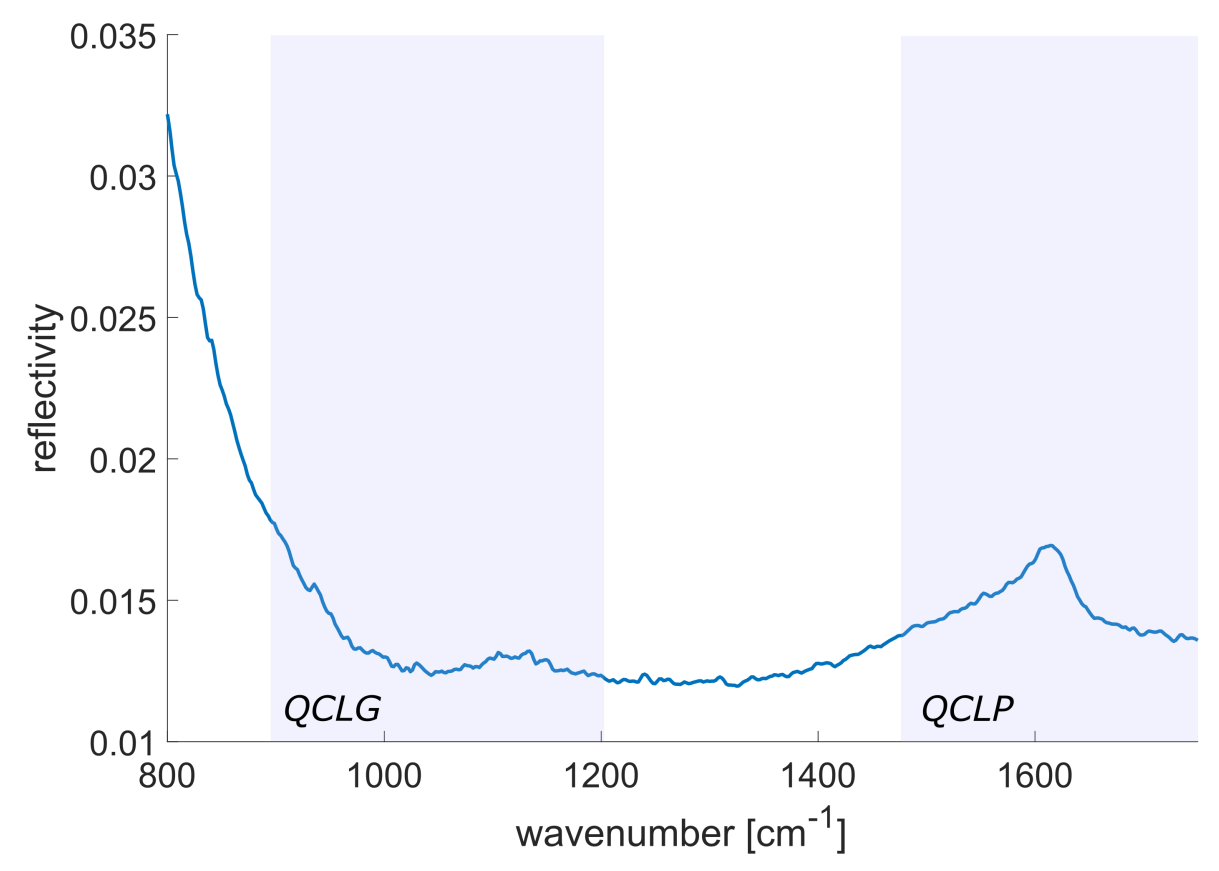

Figure 2.9.: Acquired reflectivity of anti-reflection coated diamond surface. 
- Diamond layer $\hat{\mathbf{L}}_{1}$ : The real part of the refractive index of diamond is $n_{1}=2.3776$ as measured by Edwards and Ochoa [42]. For simplicity, its spectral dependence is neglected and the complex part $k_{1}$ is set to zero (smaller than $0.2 \%$ of the complex refractive index of water $k_{2}$ )[43]. The thickness of the diamond layer $d_{1}$ is 400(50) $\mu \mathrm{m}$ (given by the manufacturer).

- Diamond-water interface $\hat{\mathbf{I}}_{12}$ : The reflection coefficient $r_{12}$ is calculated from the measured air-diamond reflectivity using Fresnel's formula (Equation 2.12): an expression for the refractive index of the anti-reflection coating (assuming the imaginary part is zero) with respect to the measured reflection coefficient $r_{01}$ is derived and the reflection coefficient $r_{12}$ is calculated based on the literature values for the complex refractive index of water given by Bertie and Lan [44].

- Water layer $\hat{\mathbf{L}}_{2}$ : The complex refractive index of water $N_{2}$ given by Bertie and Lan [44] (measured at $25^{\circ} \mathrm{C}$ ) is used together with a water layer thickness of $d_{2}=10 \mu \mathrm{m}$ (given by the thickness of the stainless steel spacer) in order to calculate $\beta_{1}$ of the layer matrix $\hat{\mathbf{L}}_{2}$.

- Water-gold interface $\hat{\mathbf{I}}_{23}$ : The optical properties of the gold layer $\left(N_{3}\right)$ are adapted from Trollmann and Pucci [45] from the measured plasma-frequency $\omega_{p}$, dielectric background $\epsilon_{\infty}$ and scattering rate $\omega_{\tau}$ (derived by Drude modeling of ellipsometry measurements) of a granular gold film on titanium on silicon $\left(\omega_{p}=67.9(26) \times 10^{4} \mathrm{~cm}^{-1}, \epsilon_{\infty}=7.0(3), \omega_{\tau}=384(4) \mathrm{cm}^{-1}\right)$ using the relation

$$
N_{3}=\sqrt{\epsilon_{\infty}-\frac{\omega_{p}^{2}}{\tilde{\nu}^{2}+i \tilde{\nu} \omega_{\tau}}} .
$$

The reflection coefficient $r_{23}$ is calculated for a pure air-gold film $\left(r_{\text {air-gold }}\right)$ and water-gold film ( $\left.r_{\text {water-gold }}\right)$ interface and then corrected with the reflection coefficient measured on the air-gold-patterned interface $\left(\tilde{r}_{\text {air-gold patterned }}\right)$ in order to compensate the effect of the holes in the gold layer. This is done at every wavenumber according to

$$
r_{23}(\tilde{\nu})=r_{\text {water-gold }}(\tilde{\nu}) \frac{\tilde{r}_{\text {air-gold patterned }}(\tilde{\nu})}{r_{\text {air-water }}(\tilde{\nu})} .
$$

\section{Temperature dependence of optical parameters}

In the following a literature overview of measurements of the most significant optical parameters in the experiment is given with a focus on their temperature dependence. Table 2.2 gives an overview of the relative possible changes during measurement and of the relative uncertainties from literature.

- Layer thickness of the water layer $d_{2}$ : The water layer thickness is determined by the stainless steal spacer ring in between the diamond and gold layer 
Table 2.1.: Thermal expansion of individual compartments of the experimental optofluidic interface, the direction of expansion indicates if the expansion leads to an increase $(+)$ or a decrease $(-)$ in water layer thickness, or if it has no direct impact $(/)$.

\begin{tabular}{|c|c|c|c|c|c|}
\hline compartment & thickness & $\begin{array}{l}\text { thermal } \\
\text { expansion } \\
\text { coefficient }\end{array}$ & expansion & direction & source \\
\hline $\begin{array}{l}\text { stainless steel sur- } \\
\text { rounding }\end{array}$ & $1.8 \mathrm{~mm}$ & $16 \times 10^{-6} 1 / \mathrm{K}$ & $28.8 \mathrm{~nm} / \mathrm{K}$ & $(+)$ & {$[46]$} \\
\hline stainless steel screw & $594 \mu \mathrm{m}$ & $16 \times 10^{-6} 1 / \mathrm{K}$ & $9.5 \mathrm{~nm} / \mathrm{K}$ & $(-)$ & [46] \\
\hline diamond window & $400 \mu \mathrm{m}$ & $1 \times 10^{-6} 1 / \mathrm{K}$ & $0.4 \mathrm{~nm} / \mathrm{K}$ & $(-)$ & {$[47]$} \\
\hline stainless steel spacer & $10 \mu \mathrm{m}$ & $16 \times 10^{-6} 1 / \mathrm{K}$ & $0.16 \mathrm{~nm} / \mathrm{K}$ & $(+)$ & {$[46]$} \\
\hline stainless steel ring & $200 \mu \mathrm{m}$ & $16 \times 10^{-6} 1 / \mathrm{K}$ & $3.2 \mathrm{~nm} / \mathrm{K}$ & $(-)$ & [46] \\
\hline silicon wafer & $200 \mu \mathrm{m}$ & $2.6 \times 10^{-6} 1 / \mathrm{K}$ & $0.5 \mathrm{~nm} / \mathrm{K}$ & $(-)$ & [48] \\
\hline silicone rings & $2 \times 125 \mu \mathrm{m}$ & & & $(/)$ & \\
\hline
\end{tabular}

which has a distinct thickness of 10(2) $\mu \mathrm{m}$. The main change in layer thickness during the experiment is caused by thermal expansion of the individual parts in the sensor (as summarized in Table 2.1): The experimental measurement chamber consists of an outer surrounding made of stainless steel and an inner part with the different layers stacked on top of each other and hold together by a holding screw. The expansion of the outer part leads to an increase in water layer thickness whereas an expansion of the inner parts leads to a decrease in layer thickness. Summing up the expansion caused by the inner parts results in an expansion of $13.6 \mathrm{~nm} / \mathrm{K}$, whereas the outer part together with the spacer ring in the measurement chamber itself increases by $29.0 \mathrm{~nm} / \mathrm{K}$. This means, that the overall stack of compartments expands by $29.0-13.6=15.4 \mathrm{~nm} / \mathrm{K}$. However some of this expansion is compensated by the elastic silicone rings which are put under pressure by the holding screw. If we assume, that the silicone ring can uphold the pressure nevertheless, the water layer thickness is uphold by the stainless steel spacer ring and a temperature change only affects the thickness of the water layer via a thermal expansion of the spacer. Its thermal expansion coefficient is about $16 \times 10^{-6} 1 / \mathrm{K}[46]$ which corresponds to an expansion of the spacer to $0.16 \mathrm{~nm} / \mathrm{K}$. Assuming a transmission measurement cell filled with water and a corresponding layer thickness of $20 \mu \mathrm{m}$ (because the beam passes the layer twice in transflection geometry), a change in temperature by $1 \mathrm{~K}$ leads to a change in absorbance of $0.0014 \%$ (assuming a wavelength of $10 \mu \mathrm{m}$ ).

- Refractive index (real part) of water $n_{2}$ : The refractive index of water in the mid infrared has been measured by Hale and Querry [24] at $25^{\circ} \mathrm{C}$, by Segelstein [49] at $25^{\circ} \mathrm{C}$, by Bertie and Lan [44] at $25^{\circ} \mathrm{C}$, by Bertie et al. [50] 
at $22^{\circ} \mathrm{C}$ and by Dowing and Williams [51] at $27^{\circ} \mathrm{C}$ as shown exemplarily for the region between 600 and $1800 \mathrm{~cm}^{-1}$ in Figure 2.10 (top). It can be seen, that the refractive index varies between the different observations in the range of \pm 0.02 and that there is no obvious correlation between refractive index and the temperature, at which the respective measurements took place (indicated by the legend).

There is not much literature on the temperature dependence of the refractive index in the mid infrared, especially, no detailed data is provided in the range from $900 \mathrm{~cm}^{-1}$ to $1200 \mathrm{~cm}^{-1}$ and $1500 \mathrm{~cm}^{-1}$ to $1750 \mathrm{~cm}^{-1}$. Pinkley et al. [52] provide data for the wavenumber region below $820 \mathrm{~cm}^{-1}$ and between $1620 \mathrm{~cm}^{-1}$ and $1690 \mathrm{~cm}^{-1}$ measured at five different temperatures between $1{ }^{\circ} \mathrm{C}$ and $50{ }^{\circ} \mathrm{C}$. Using this data the change in $n_{w}$ at every given wavenumber can be extracted by a linear fit. This is shown in Figure 2.10 (bottom) in green with the error bars indicating the standard error of the fit. The average change in refractive index over the shown wavenumber range is $-9.4(50) \times 10^{-4} 1 /{ }^{\circ} \mathrm{C}$ indicating that, overall, the refractive index decreases with an increase in temperature. However, spectrally there is a high change in temperature dependence observable between $-2 \times 10^{-4} 1 /{ }^{\circ} \mathrm{C}$ and $-1.6 \times 10^{-3} 1 /{ }^{\circ} \mathrm{C}$.

In comparison to the variation of the literature values of refractive index given by different groups (standard deviation 1.6\%), the variation caused by temperatue is two orders of magnitude smaller $\left(0.08 \%{ }^{\circ} \mathrm{C}^{-1}\right)$.

- Absorption coefficient $\alpha_{2} /$ complex refractive index $k_{2}$ of water: The complex refractive index $k$ correlates linearly with the absorption coefficient $\alpha$ as shown in Equation 2.15. As it is more common to give the absorption coefficient in literature, we will discuss this parameter in the following, but the findings can be directly applied to $k$ using Eq. 2.15. Several groups have measured the value with respect to the wavenumber using FTIR spectroscopes in combination with transmission setups or ATR setups. In Figure 2.11 (top) the most common data is shown in the biological fingerprint region of the infrared [24, 44, 51, 54-57]. The data is consistent between the different measurements. In order to estimate the variance between the different literature values, the average standard deviation $\overline{\operatorname{STD}}(\alpha)$ as well as the average absolute value $\bar{\alpha}$ of absorbance is calculated. This leads to $\overline{\operatorname{STD}}(\alpha)=12 \mathrm{~cm}^{-1}$ and $\bar{\alpha}=282 \mathrm{~cm}^{-1}$ and $\overline{\operatorname{STD}}(\alpha)=15 \mathrm{~cm}^{-1}$ and $\bar{\alpha}=586 \mathrm{~cm}^{-1}$ for the spectral regions of QCLG $\left(900 \mathrm{~cm}^{-1}\right.$ to $1200 \mathrm{~cm}^{-1}$, references $\left.[24,44,51,56,57]\right)$ and QCLP $\left(1500 \mathrm{~cm}^{-1}\right.$ to $1750 \mathrm{~cm}^{-1}$, references $\left.[24,44,51,56]\right)$, respectively.

The most relevant impact that can change the absorbance value of water in our experiment (approximately constant pressure and constant volume) is the temperature. The influence of temperature on the absorbance of water has been investigated by simulations [58] as well as experimentally [57, 59, 60]. Jensen et al. [57] provide a measured data set of the decadic absorptivity in the spectral region from $960 \mathrm{~cm}^{-1}$ to $3000 \mathrm{~cm}^{-1}$ at temperatures between $30{ }^{\circ} \mathrm{C}$ 
to $42{ }^{\circ} \mathrm{C}$. By assuming a linear relationship of absorptivity with temperature at every respective wavenumber a linear regression is done and the resulting spectral temperature dependence is shown in green in Figure 2.11 (bottom) with error bars indicating the standard error of the regression. For comparison also the absolute values of the absorption coefficient measured by the group are shown in the overview in Fig. 2.11 (top, green crosses). In most parts of the spectrum the absorptivity is reduced by an increase of temperature and the overall change with temperature is quite small in the range between $0.21 /\left(\mathrm{cm}^{\circ} \mathrm{C}\right)$ to $-1.51 /\left(\mathrm{cm}^{\circ} \mathrm{C}\right)$. Further, a strong wavenumber dependence is observable.

- Reflection coefficients of the antireflective coating on diamond $r_{12}, r_{23}$ : The reflectivity of the air-diamond interface with additional antireflective coating is below $2 \%$ per interface in nearly all parts of the spectrally relevant region (measured in reflection with a FTIR spectrometer, see Figure 2.9). In order to get a high degree of transmission, antireflective coatings are commonly based on coherence effects originating from different thin layers with different refractive indices. A temperature change could have an impact on this effect by a thermal expansion of the layers. However, as there is no detailed information given about the coating, this effect cannot be quantified. Additional uncertainties of the measured reflectivity caused by a difference in polarization as well as a difference in the angle of incidence between the transflection setup and the used FTIR spectrometer can be neglected under small angles of incidence.

- Thickness of diamond window $d_{1}$ : The absolute thickness of the diamond windows is given by the manufacturer to be $400(50) \mu \mathrm{m}$, whereby the error is the manufacturing variance from one piece to another. The parallelism of the two surfaces is specified to be better than $1^{\prime}$. The thickness is obviously dependent on a temperature change by thermal expansion, however, the thermal expansion coefficient of diamond is as small as $1 \times 10^{-6} 1 / \mathrm{K}$ which leads to an negligible expansion of $0.4 \mathrm{~nm} / \mathrm{K}$.

- Refractive index (real part) of diamond $n_{1}$ : Edwards and Ochoa [42] measured the spectral dependence of the refractive index of diamond and showed, that in the spectral range from $5 \mu \mathrm{m}$ to $11 \mu \mathrm{m}$ the value stays constant at a value of $2.376 \pm 0.001$. The temperature dependence of the refractive index has only been measured in the visible as well as in the long wavelength region [61]. In both regions the change per Kelvin is around $10 \times 10^{-6} 1 / \mathrm{K}$ which corresponds to a relative change as low as $0.0004 \% / \mathrm{K}$.

- Absorption coefficient $\alpha_{1} /$ complex refractive index $k_{1}$ of diamond: The absorbance of CVD diamond in the infrared region has been measured by Mollart and Lewis [43] and is shown in Fig. 2.12. In the relevant wavenumber region of our experiment $\left(900 \mathrm{~cm}^{-1}\right.$ to $\left.1750 \mathrm{~cm}^{-1}\right)$ an (exponential) absorption coefficient of about $0.1 \mathrm{~cm}^{-1}$ is observable (at $25^{\circ} \mathrm{C}$ ). Compared to the 
high absorption of the water layer in the sensor it is reasonable to neglect the absorption of diamond. Towards higher wavenumbers above $1600 \mathrm{~cm}^{-1}$ the multiple-phonon absorption becomes prominent, however around $1750 \mathrm{~cm}^{-1}$ which constitutes the upper limit of our measurement range, the (exponential) absorption coefficient is still $1 \mathrm{~cm}^{-1}$ which is still as low as $0.2 \%$ of the absorption coefficient of water at this wavenumber.

In Fig. 2.13 the temperature dependence of absorbance at $\tilde{\nu}=1000 \mathrm{~cm}^{-1}$ is shown for a diamond sample of a comparable grade to the one used in the experiments [43]. The change in absorbance at temperature changes of a few ${ }^{\circ} \mathrm{C}$ (for temperatures below $100{ }^{\circ} \mathrm{C}$ ) is negligibly small.

- Reflection coefficient of the water-gold interface $r_{23}$ : The main uncertainty in the reflectivity is based on the measurement of the reflectivity of the hole-patterned gold interface. Depending on the position of the measurement the reflectivity varied by $3 \%$ (obtained by 100 measurements at different positions on the wafer).

- Beam properties of the laser beam $D_{\text {beam }}, x_{c e n t e r}, f, g$ : The geometric properties of the beam as well as the position should not change during measurement, e.g. when changing the temperature of the measurement chamber. However, it has a total error based on the manufacturers information about the beam waist, the measurement of the position of the beam on the lens, the error of the focal length of the lens, and the deviation of the beam shape from an ideal gaussian beam.

Table 2.3 gives an overview on the used absolute input parameters for the simulations.

\section{Simulation of the transflection signal}

Using the here introduced calculation together with the laser's spectral intensity profile $I_{\text {incident }}$ the reflected signal $I_{\text {reflected }}$ can be calculated. An example is shown in Figure 2.14. Further the transflectance is calculated according to Equation 2.6 for glucose at different layer thicknesses and shown in Figure 2.15.

\subsubsection{Optimum layer thickness}

To extract the perfect sensor setup parameters, the optimum water layer thickness for a maximum glucose transflectance signal is investigated. This question comes with a trade-off between an increase in glucose-specific absorption and an overall decrease in total signal (with increasing layer thickness). In the following a comparison between optimum layer thickness in transmission and transflection geometry is given. 
Table 2.2.: Overview on the uncertainties of the individual contributions to the overall signal, the values are calculated with respect to the absolute value of each individual parameter. The absorptivity of the diamond window $\alpha_{1}$ is completely neglected in the simulations. ${ }^{*}$ ) assuming silicone upholds pressure, $\left.{ }^{* *}\right)$ assuming silicone is not elastic.

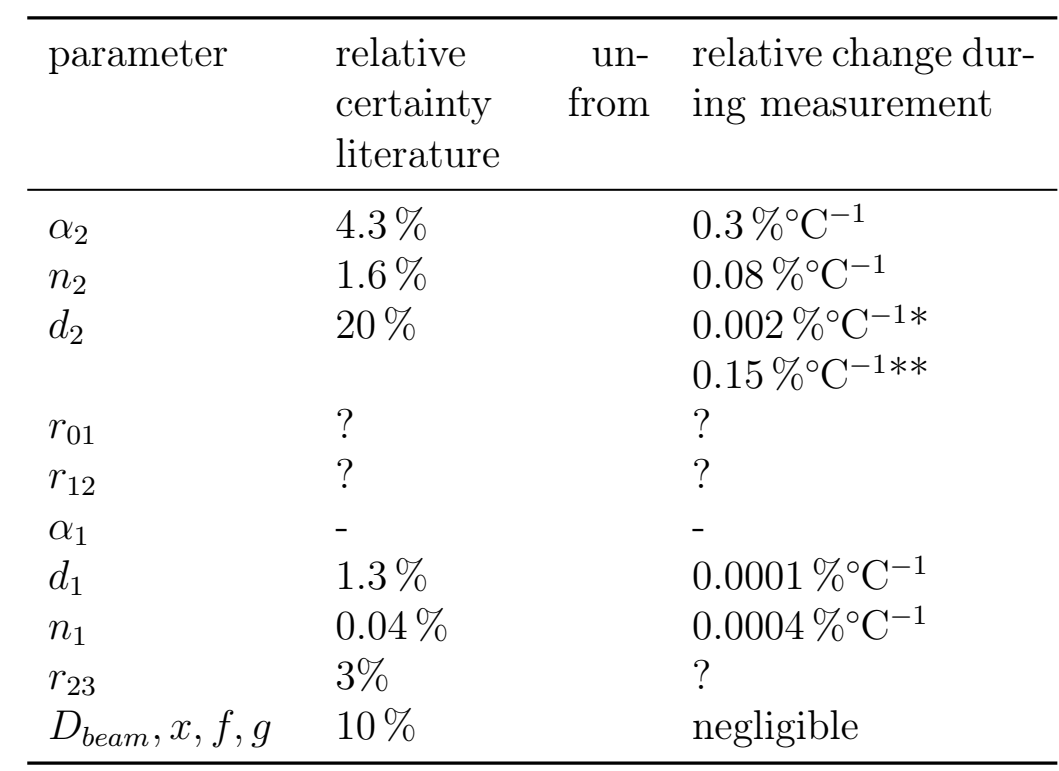

The intensity of a beam transmitted through a layer of thickness $d$ of an aqueous mixture of substance $i$ can be described according to Beer-Lambert law

$$
I_{\text {substance }}=I_{0} e^{-\left(\alpha_{w}+\sum_{i} \alpha_{i}\right) d}
$$

with $\alpha_{w}$ being the absorption coefficient of water, $\alpha_{i}$ being the absorption coefficient of the substance $i$ (additional effects from the windows of a transmission cell are neglected for simplicity). To find the optimum layer thickness, the difference between the signal gained from pure water $I_{w, \text { transm }}$ and the signal gained from the aqueous

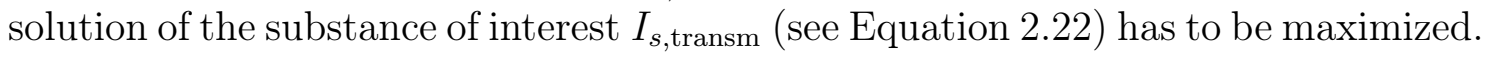

$$
\Delta I_{\text {transm }}=I_{w}\left(d_{2, \text { transm }}\right)-I_{s}\left(d_{2, \text { transm }}\right)=I_{0}\left(e^{-\alpha_{w} d_{2, \text { transm }}}-e^{-\left(\alpha_{w}+\sum_{i} \alpha_{i}\right) d_{2, \text { transm }}}\right)
$$

As shown e.g. by Vrancîć [22] this leads to an optimum path length of $d_{\text {optimum }} \rightarrow$ $1 / \alpha_{w}$ when $\alpha_{w}>>\alpha_{s}$. The equivalent calculation to Equation 2.22 can be done for the transflection signal according to

$$
\Delta I_{\text {transfl }}=I_{\text {refleceted,water }}\left(d_{2}\right)-I_{\text {reflected,water+substance }}\left(d_{2}\right)
$$

assuming a spectrally constant absorbing substance with an absorption coefficient of $\alpha=0.2$ and the same geometric and optical properties as in the simulations introduced in Sec. 2.1.4. The result is shown in Figure 2.16 at a wavenumber of 
Table 2.3.: Overview on the input parameters used for the simulations of the signal $I_{3 D}$ either with or without assumed temperature dependence. If not noted otherwise the data is given by the manufacturer.

\begin{tabular}{|c|c|}
\hline Parameter & Data used \\
\hline$k_{2}(\tilde{\nu}, T)$ & $\begin{array}{l}k_{2}(\tilde{\nu}, T)=k_{2, \operatorname{Bertie}}\left(\tilde{\nu}, 25^{\circ} \mathrm{C}\right)+\left(\frac{d k_{2}(\tilde{\nu})}{d T}\right) \text { Jensen } \times\left(T-25^{\circ} \mathrm{C}\right) \text { with }\left(\frac{d k_{2}(\tilde{\nu})}{d T}\right)_{\text {Jensen }} \\
\text { being calculated from absorbance data given by Jensen et al. [57] }\end{array}$ \\
\hline$D_{\text {beam }}$ & $D_{\text {beam }}=1.77 \mathrm{~mm}$ \\
\hline$d_{1}$ & $d_{1}=(400 \pm 50) \mu \mathrm{m}$ \\
\hline$d_{2}(T)$ & $\begin{array}{l}\text { Calculated by } d_{2}(T)=d_{2}\left(30^{\circ} \mathrm{C}\right)+\left(T-30^{\circ} \mathrm{C}\right) \times 0.0054 \mu \mathrm{m} /{ }^{\circ} \mathrm{C} \text { with } d_{2}\left(30^{\circ} \mathrm{C}\right)= \\
10 \mu \mathrm{m} \text { derived experimentally }\end{array}$ \\
\hline$f$ & $f=25 \mathrm{~mm}$ \\
\hline$g$ & $\begin{array}{l}\text { Gaussian intensity distribution of the beam is acquired by } g\left(D_{\text {beam }}, x_{0}, x\right)= \\
\frac{1}{\left(D_{\text {beam }} / 2\right) \sqrt{2 \pi}} \exp \left\{\left(\frac{-\left(x-x_{0}\right)^{2}}{2\left(D_{\text {beam }} / 2\right)^{2}}\right)\right\} \text { and integrated from }-3 \sigma \text { to } 3 \sigma \text { in order to } \\
\text { gain } 99.7 \% \text { of the intensity }\end{array}$ \\
\hline$I_{\text {incident }}(\tilde{\nu})$ & Measured gain profiles of the QCLs \\
\hline$n_{1}$ & $n_{1}=2.3778$ given by Phillip and Taft $[62]$ \\
\hline$n_{2}(\tilde{\nu}, T)$ & $\begin{array}{l}n_{2}(\tilde{\nu}, T)=n_{2, \operatorname{Bertie}}\left(\tilde{\nu}, 25^{\circ} \mathrm{C}\right)-\left(T-25^{\circ} \mathrm{C}\right) \times 0.001^{\circ} \mathrm{C}^{-1} \text { with } n_{2, \operatorname{Bertie}}\left(\tilde{\nu}, 25^{\circ} \mathrm{C}\right) \\
\text { given by Bertie and Lan [44] and the temperature dependence of }-0.001{ }^{\circ} \mathrm{C}^{-1} \\
\text { adapted from Pinkley et al. [52] }\end{array}$ \\
\hline$r_{01}, r_{12}$ & $\begin{array}{l}r_{01} \text { derived by reflexion measurement in air with FTIR, } r_{12} \text { calculated from } r_{01} \\
\text { using Fresnel formula as described in text }\end{array}$ \\
\hline$r_{23}$ & $\begin{array}{l}\text { Derived by adapted reflexion measurement done in air with FTIR as described } \\
\text { in text }\end{array}$ \\
\hline$x_{\text {center }}$ & $x_{\text {center }}=3.5 \mathrm{~mm}$, manually measured \\
\hline
\end{tabular}


$1035 \mathrm{~cm}^{-1}$ as well as on average in the spectral range of QCLG and QCLP. Further, the corresponding $\left(d_{2, \text { transfl }}=2 d_{2 \text {,transm }}\right)$ optimum curve for transmission is shown in green. All curves that are calculated at wavenumbers around $1000 \mathrm{~cm}^{-1}$ show an optimum in layer thickness around $8 \mu \mathrm{m}$. The curve of the region of QCLP shows an optimum at smaller thicknesses around $5 \mu \mathrm{m}$. This difference is caused by the higher absorption coefficient of water in the spectral region of QCLP. The overall shape of curves measured in transflection is similar to the ones measured in transmission geometry. However, an oscillating modulation with water layer thickness is observable in the case of transflection. This is presumably caused by interference effects of the individual electromagnetic waves generated at the boundaries in the setup as described in Sec. 2.1.4.

\subsubsection{Data processing}

All data processing is implemented in Matlab (The MathWorks Inc. USA) and Python (Python Software Foundation, USA).

\section{Warping}

Prior to data processing the spectra of 4 sweep scans are averaged. Due to small differences in scanning speed (caused by mechanical imperfections during rotation of the laser's grating or triggering issues [19]) the spectra show small variances from scan to scan (see Figure 2.17 A). When calculating the transflectance and referencing different spectra, this can be misinterpreted as a change in transflectance. In order to align the single spectra prior to averaging a so-called correlation optimized warping (COW) algorithm is used as it is commonly applied on chromatographic and mass spectrometry data [63]. In this algorithm the spectra are split into small sections with a distinct section length and then compressed or stretched in order to maximize the correlation between the individual spectra. The maximum wavenumber of stretching or compressing can be set as limit in order to stay in the physically realistic variation range. Using this method the spectral peaks get neither spectrally distorted nor their absolute peak heights change and thus the result can still be reliably interpreted [63, 64].

In our experiment the acquired spectra contain a systematic oscillation caused by interference effects at a $\mathrm{BaF}_{2}$ window integrated in front of the detectors. This wavelength-dependent structure can be used for the alignment. COW is done with a matlab function provided by Tomasi et al. [65] with a section length of $5 \mathrm{~cm}^{-1}$ and a maximum range of warping of $1.25 \mathrm{~cm}^{-1}$. Figure 2.17 shows an example of the spectra before and after application of COW. As the spectral resolution of the lockin detection system is about $5 \mathrm{~cm}^{-1}$, all spectra are spectrally binned over $4 \mathrm{~cm}^{-1}$ after warping in order to reduce overall data size. 


\section{PLS Analysis}

A spectrum is a high dimensional data set where the information of interest (e.g. the concentration of a specific substance) often is contained in a specific part of this dataset, e.g. a specific spectral range in the spectrum or in a specific relative deviation between different regions in the spectrum. Multivariate data analysis methods serve as a tool to find these specific parts and to reduce dimensionality of the dataset without substantially reducing the amount of information. The most commonly used methods for the determination of a concentration of a substance of interest in a MIR spectrum are a regression based on principal component analysis (PCR) and a partial least square analysis (PLS) [66, 67]. These methods have in common that they are first trained on a data set with known concentrations in order to predict the concentrations for an unknown spectrum.

In this thesis PLS analysis also known as 'projection to latent structures' is used for this purpose (if not noted otherwise). It is based on a transformation of coordinates such that the maximum variance between the spectra and the maximum variance between the concentrations are correlated to each other. In mathematical view: if the spectra are contained in a data matrix $\hat{D}$ and the concentrations are contained in a matrix $\hat{C}$ the equations

$$
\begin{aligned}
& \hat{D}=\hat{T} \cdot \hat{P}^{T}+\hat{E} \\
& \hat{C}=\hat{U} \cdot \hat{Q}^{T}+\hat{F}
\end{aligned}
$$

show the representation in the transformed coordinates with $\hat{T}$ and $\hat{U}$ being the projections of $\hat{D}$ and $\hat{C}$ in the new coordinates. Hereby $\hat{E}$ and $\hat{F}$ are error terms and $\hat{P}$ and $\hat{Q}$ are the transformation matrices. These are chosen by maximization of the covariance between $\hat{T}$ and $\hat{U}$, such that the first coordinate contains the highest correlated variance i.e. the highest amount of information regarding the concentration change and the spectral change. The next coordinate contains the second highest amount and so on. After the transformation matrices are found by the so called 'calibration' step, this transformation can be applied onto spectra with unknown concentrations in order to predict the concentrations. By only taking into account a distinct number of coordinates a conscious reduction of non relevant information or noise can be achieved. The best number of coordinates is usually chosen by minimization of the root mean squared error of cross validation (RMSECV) as will be shown in the following.

Prior to PLS analysis the data is usually transformed at every wavenumber by subtraction of the mean and dividing by the standard deviation (STD). This leads to an equal weighting of every wavenumber. In our experiment, however, due to the experimental setup the signal-to-noise is not equal at all wavenumbers because the lasers emission profile (as shown in Fig. 2.6) is not homogeneous with respect to wavenumber. Thus the wavenumbers are not equally reliable. To include this effect, the data is scaled by the 'experimental measurement error' (EME) at every wavenumber instead of the STD. The EME is calculated by the average standard 
deviation of the individual scans of a single measurement (normally 4 scans) with respect to wavenumber. This way, data at wavenumbers with higher signal-to-noise ratio are weighted stronger than data on wavenumbers with a low signal-to-noise ratio. The effect of scaling with the EME compared to with the STD (as it is normally done) is shown in Fig. 2.18. When scaling with the EME a lower RMSECV is gained and a smaller amount of latent variables is needed.

PLS analysis is done in Python using the scikit-learn package (Python Software Foundation, USA).

\section{Cross validation and important quantification paramaters}

For a reliable application of PLS analysis, the measured data set is split up into two parts: one to train the PLS analysis (training set) and one to predict the concentration on (test set). In order to quantify how good this analysis is, the root mean squared error from the predicted concentrations $C_{i \text {,predicted }}$ and the reference conentrations $C_{i \text {,ref }}$ in the test set is calculated according to

$$
R M S E=\sqrt{\frac{\sum_{i=1}^{n}\left(C_{i, \text { ref }}-C_{i, \text { predicted }}\right)^{2}}{n}}
$$

with $n$ being to total number of predictions in the test set. The reference concentration $C_{i, r e f}$ is the concentration used when mixing the solutions and is seen as a gold standard in the calculations. After this calculation, the training and test set are permuted such that a different part of the data is assigned to training and test set. The overall mean of the RMSEs of all possible permutations is called the RMSE of cross validation (RMSECV). According to the split there are different cross validations possible, here we either split the single measurement cycles $\left(\mathrm{RMSECV}_{\text {cycle }}\right)$ or the measurement days ( $\left.\mathrm{RMSECV}_{\text {day }}\right)$.

This cross validation mechanism is very important in order to prevent overfitting of the PLS analysis on the specific data. This means if we use too many latent variables the prediction is very good on the data set it is trained with, however when predicting a new dataset e.g. taken at another day or setup, the predictions are worse. This effect is shown exemplarily in Figure 2.19. The violet curve indicates a PLS analysis with prediction on the training set and the blue curve indicates a cross validated PLS analysis (prediction on the test set).

\section{Parkes error grid}

Parkes error grid is a schematic to plot the predicted glucose concentration versus the real concentration. It was suggested by Parkes et al. [68] and is widely used for the characterization of glucose monitoring systems for diabetes management. The grid is subdivided into different areas indicating the medical relevance of the values for diabetes patients. The areas are indicated by the letters $\mathrm{A}$ to $\mathrm{E}$ where $\mathrm{A}$ is the least and $\mathrm{E}$ is the most risky area. 


\section{Evolutionary algorithm for the selection of specific wavenumbers}

For selection of the most important wavenumbers with respect to glucose prediction, an evolutionary algorithm [16] can be applied: After setting the number of wavenumbers to $N, 400$ packages of $N$ wavenumbers are randomly picked out of the lasers wavenumber range. On every package a PLS analysis with $N$ latent variables is done and the packages are ordered according to their RMSECV. From this result, the best $40 \%$ of the packages are used together with $20 \%$ random mutation of these packages, as well as $40 \%$ new randomly built packages. Then a PLS analysis is done on the new set of packages and the next iteration starts by arranging the new subset in the same way, and so on. This is done until the RMSECV is does not significantly change anymore.

\subsubsection{Measurements}

The aqueuous solutions of glucose and other substances are prepared by solving the right amount of glucose ( $\alpha$-D-glucose, Merck KGaA, Germany), fructose (49811, Carl Roth GmbH + Co. KG, Germany), maltose (M5885, Sigma Aldrich, Germany), lactose (8921.1, Carl Roth GmbH + Co. KG, Germany), galactose (4987.1, Carl Roth GmbH + Co. KG, Germany), maltodextrin (419680, Sigma Aldrich, Germany) and albumin (A7906, Sigma Aldrich, Germany) in deionized water.

The spectral measurements for the different solutions are done in different measurement cycles consisting of measurements of 6 to 11 various concentrations in a random order. Each measurement series consisted of 6 to 8 measurement cycles. In every cycle, if not noted otherwise, every third measurement is an additional measurement of deionized water for referencing. The single measurements are done by pumping the respective solution into the fluidic reservoir and starting the spectral measurement after a compensation time of 3 minutes. Each spectral measurement consists of 4 laser sweep scans with QCLG (from $900 \mathrm{~cm}^{-1}$ to $1200 \mathrm{~cm}^{-1}$ ) and (for some measurements, as noted in results) 4 additional laser sweep scans with QCLP (from $1500 \mathrm{~cm}^{-1}$ to $1750 \mathrm{~cm}^{-1}$ ). The lock-in detection is done with a time constant of $30 \mathrm{~ms}$, a sensitivity of $500 \mathrm{mV}$ (QCLG) and $200 \mathrm{mV}$ (QCLP), an AC coupling and a lowpass filter slope of $24 \mathrm{~dB} /$ Oct with dynamic reserve set to 'normal'. This leads to an overall lock-in integration time (time after which the individual signals are independent from each other) of $300 \mathrm{~ms}$. The measurements are done using the setup introduced in Section 2.1.2 without the optional filtering membrane in the opto-fluidic interface. 


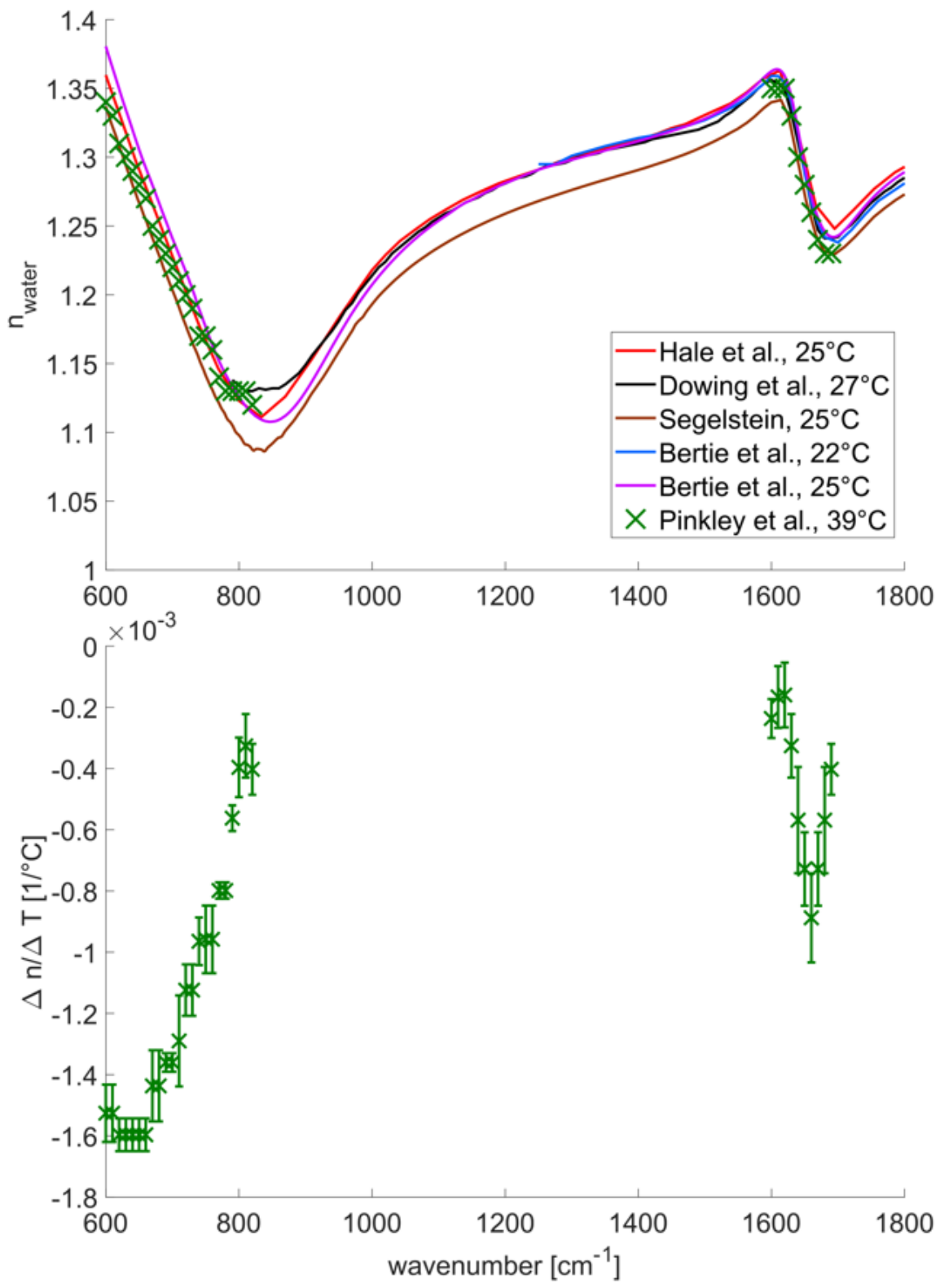

Figure 2.10.: Overview on temperature dependence of refractive index. Top: For comparison the absolute values of the refractive index of water given by [24, 44, 49-51, 53] at different temperatures and the ones given by Pinkley et al. [52] at $39^{\circ} \mathrm{C}$ (green). Bottom: Temperature dependence calculated by a linear fit on the data provided by Pinkley et al. [52] and the standard error of the slope shown as error bars. 

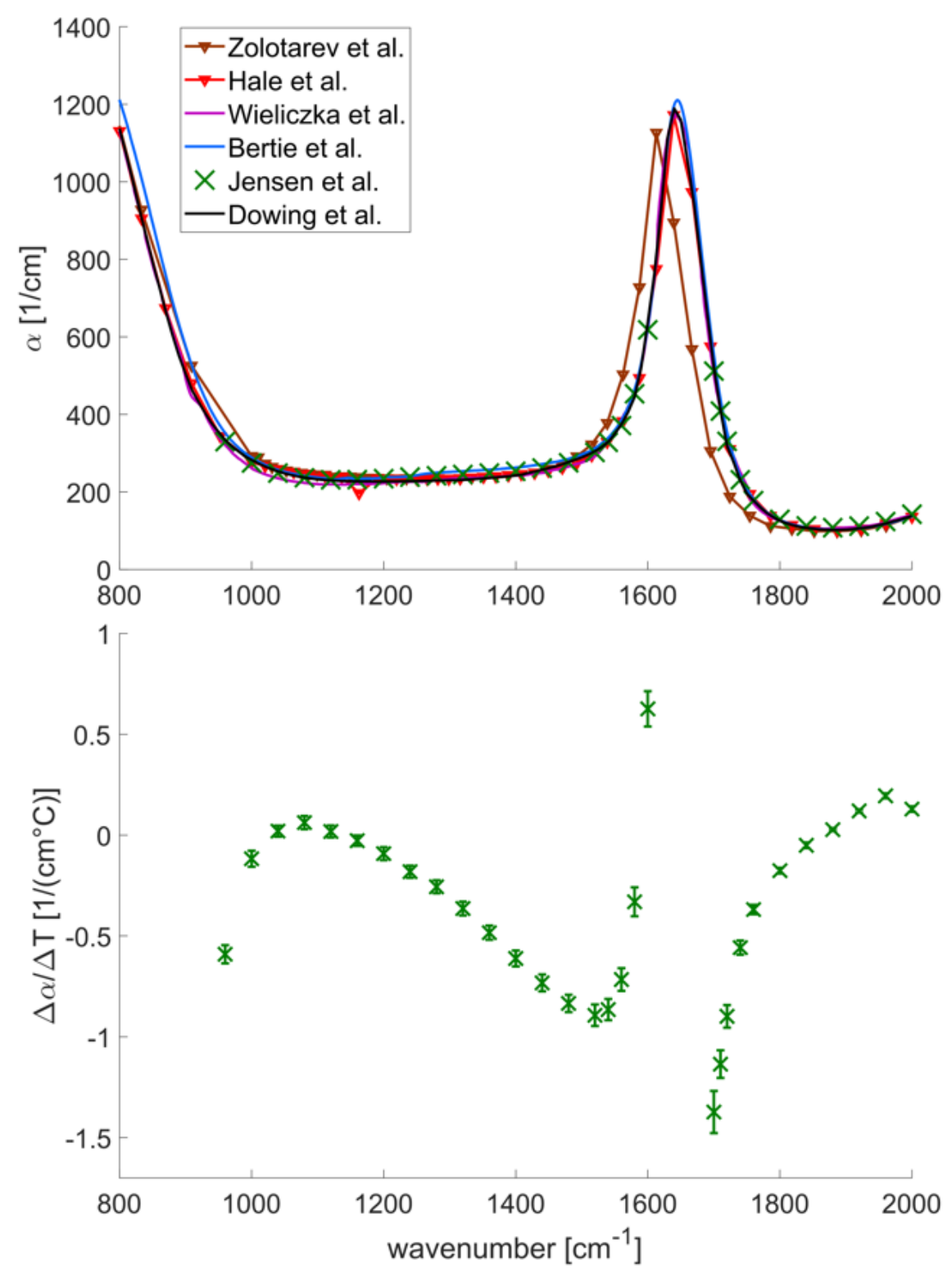

Figure 2.11.: Overview on the temperature dependence of (decadic) absorption coefficient of water $\alpha_{w}$. Top: Overview on absolute values of the absorption coefficient measured by $[24,44,51,53,56]$, the values given by Jensen et al. [57] at a temperature of $32{ }^{\circ} \mathrm{C}$ are shown in green. Bottom: Change in absorption coefficient with temperature calculated by a linear fit at each wavenumber using data given by Jensen et al. [57]. The error bars indicate the standard error of the respective fit. 


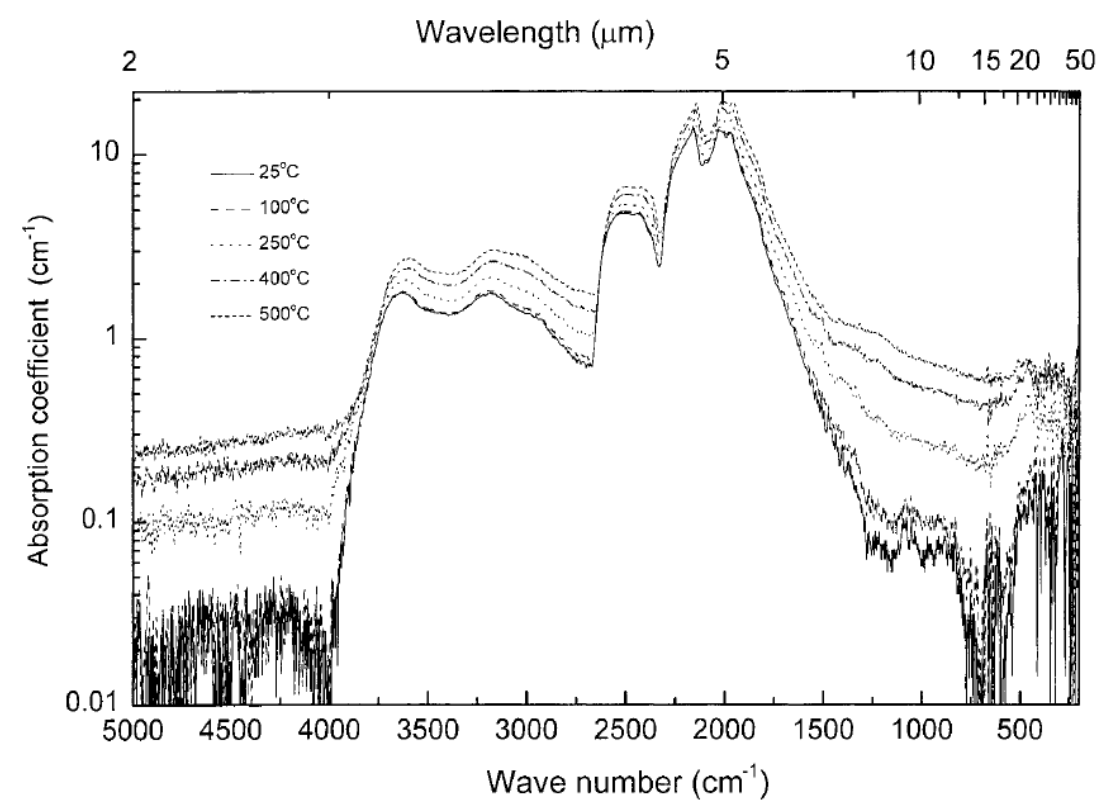

Figure 2.12.: Absorption coefficient (exponential) of a $0.5 \mathrm{~mm}$ thick CVD diamond measured at different temperatures. Image taken from Mollart and Lewis [43].

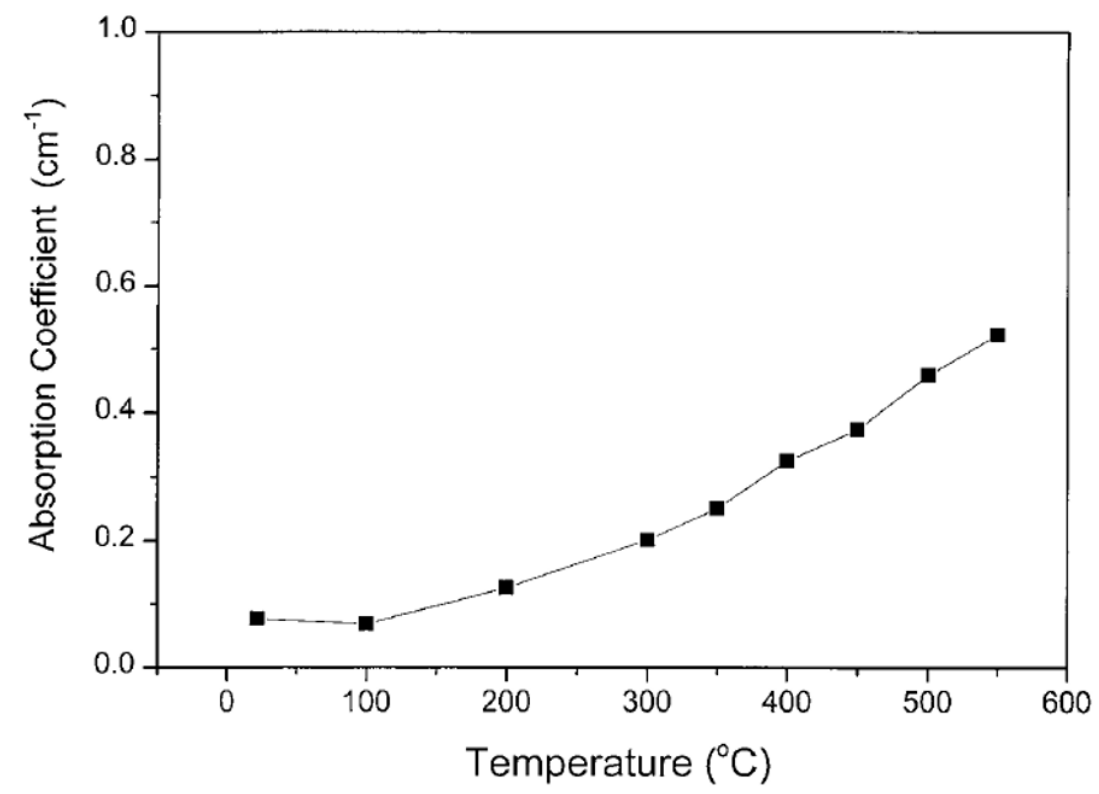

Figure 2.13.: Temperature dependence of absorption coefficient at $\tilde{\nu}=1000 \mathrm{~cm}^{-1}$ calculated from transmission of a $1 \mathrm{~mm}$ thick optical grade CVD diamond measured by Mollart and Lewis [43]. Image taken from Mollart and Lewis [43]. 


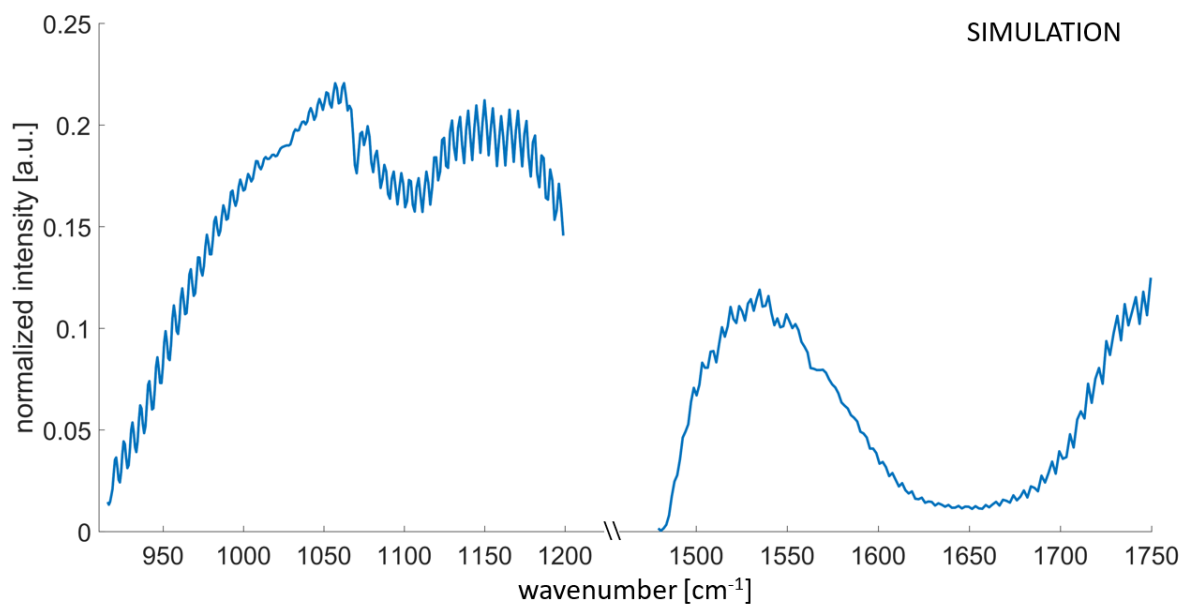

Figure 2.14.: Result of simulation of the reflected signals $I_{3 D}$ shown in the spectral region of QCLG and QCLP. 


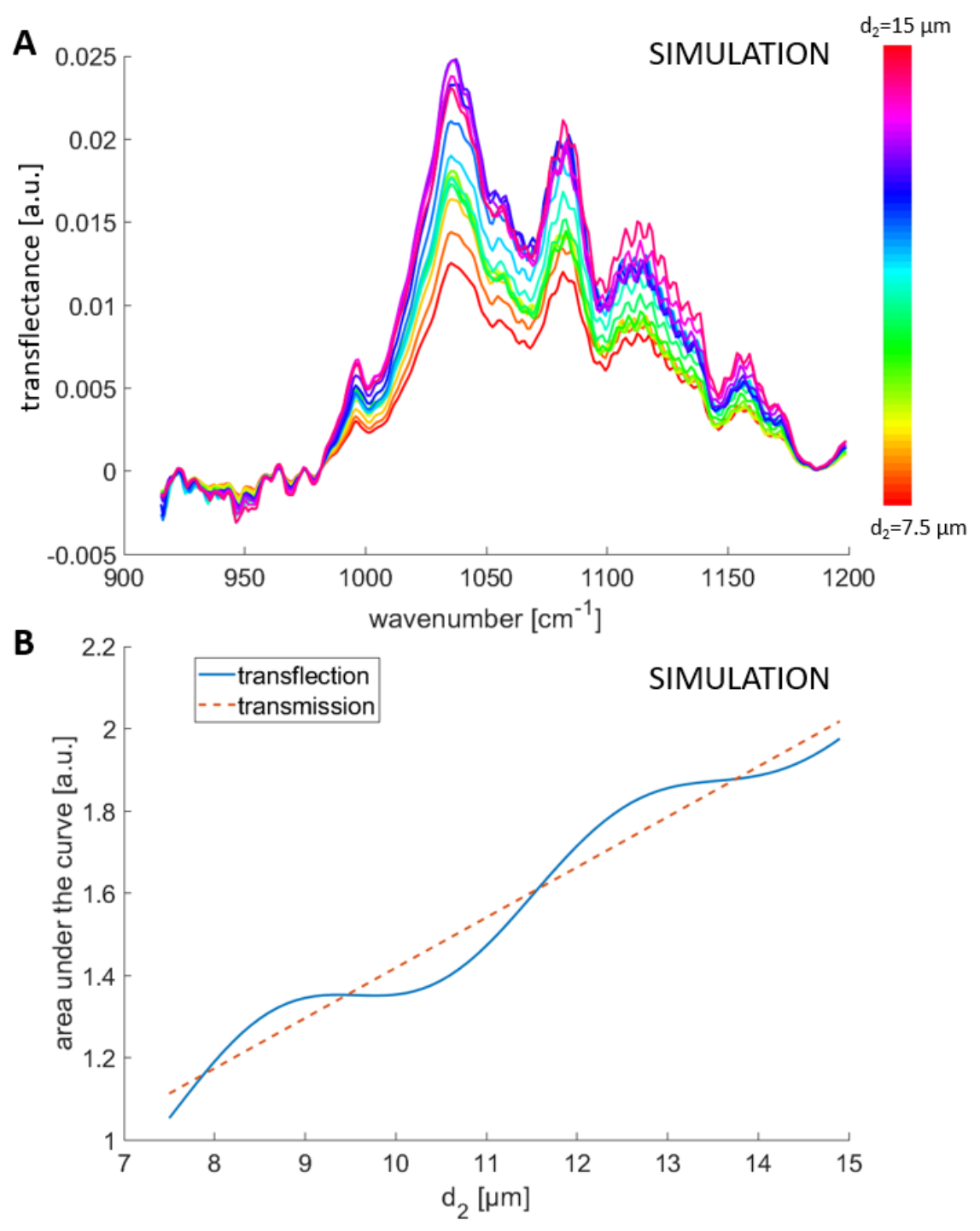

Figure 2.15.: A Transflectance spectra of $500 \mathrm{mg} / \mathrm{dL}$ glucose simulated for a transflection geometry with various layer thicknesses between $7.5 \mu \mathrm{m}$ to $15 \mu \mathrm{m}$ (indicated by color); B: Area under the transflectance curves vs. layer thickness $d_{2}$. From a measurement in transmission a linear dependence is expected as indicated by the dashed line. However, for a measurement in transflection a periodic modulation of the area under the curve with respect to layer thickness is present. This is caused by coherence effects as derived in Sec. 2.1.4 


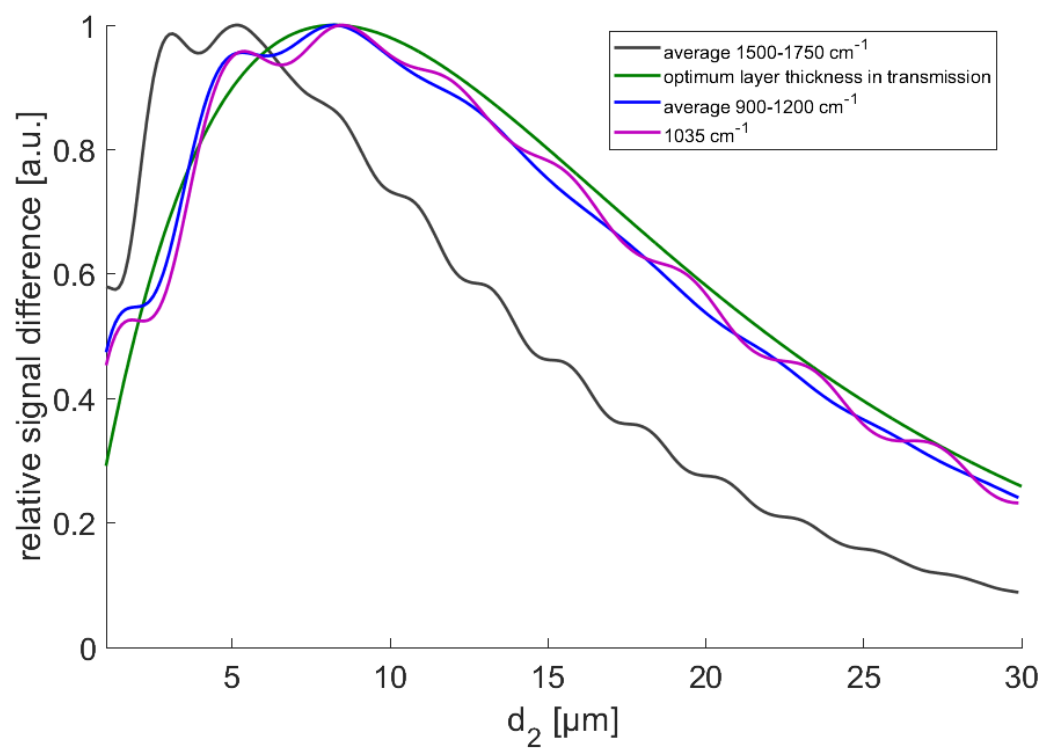

Figure 2.16.: Signal difference $I_{\text {reflected,water }}-I_{\text {reflected,water+substance }}$ with respect to the water layer thickness $d_{2}$. A constant absorbance of $\alpha_{\text {substance }}=0.2$ was assumed at all wavenumbers. The curves show the spectral average over the region of QCLG (green) and QCLP (violet) as well as the curve at $1035 \mathrm{~cm}^{-1}$. Further the calculated theoretical curve for a measurement in transmission is shown in blue, in this case the thickness was divided by two to adapt to the double passing of the liquid in transflection geometry $\left(d_{2, \text { transmission }}=2 d_{2}\right)$. The signal difference of transflection measurements show a periodic modulation that presumably comes from the interference effects occuring in transflection geometry. 


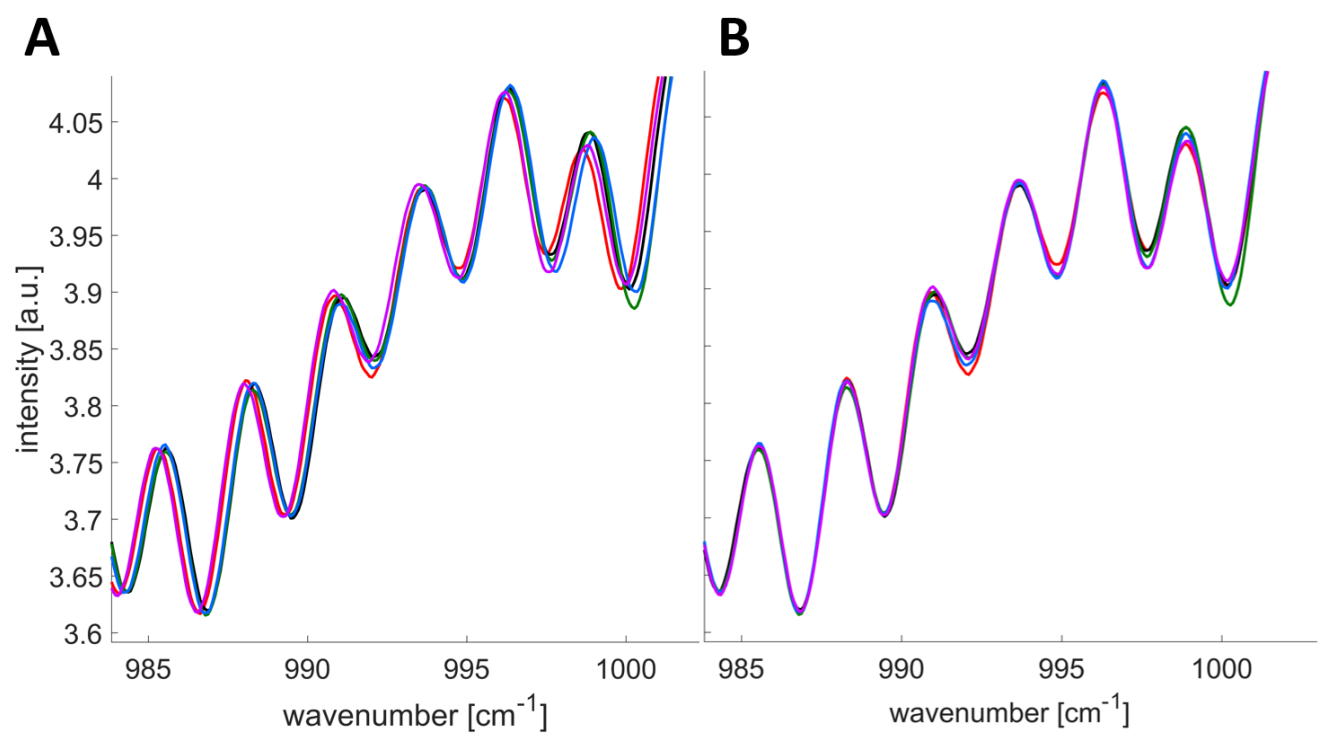

Figure 2.17.: Effect of correlation optimized warping on the single scans. A: Intensities of single laser sweep scans with respect to wavenumber as they are measured. As the relative shift in wavenumber varies in different wavenumber regions, a stretching or compressing of the data with respect to wavenumber needs to be done. B: intensities of single scans after correlation optimized warping. 


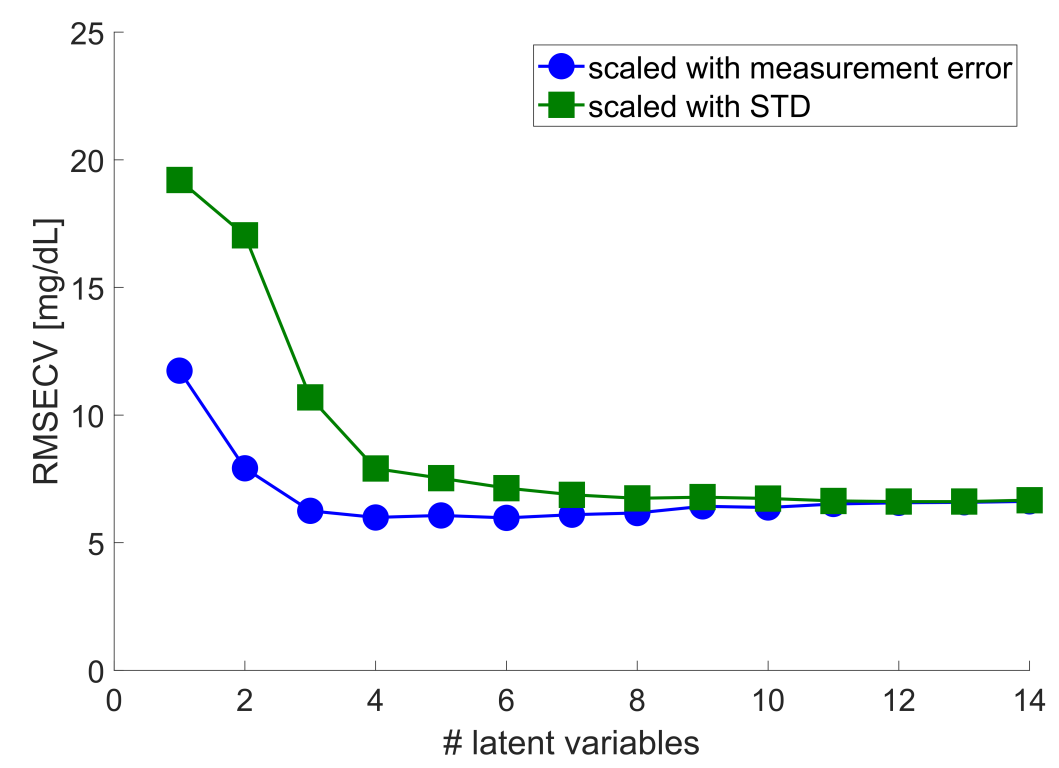

Figure 2.18.: RMSECV from a dataset with its individual spectra scaled with the standard deviation (STD) of the whole training set at every wavenumber (green) and scaled with the experimental measurement error (EME) at every wavenumber (blue). The minimum of RMSECV is achieved with less latent variables when scaling with the EME, also a better absolute value of RMSECV can be gained.

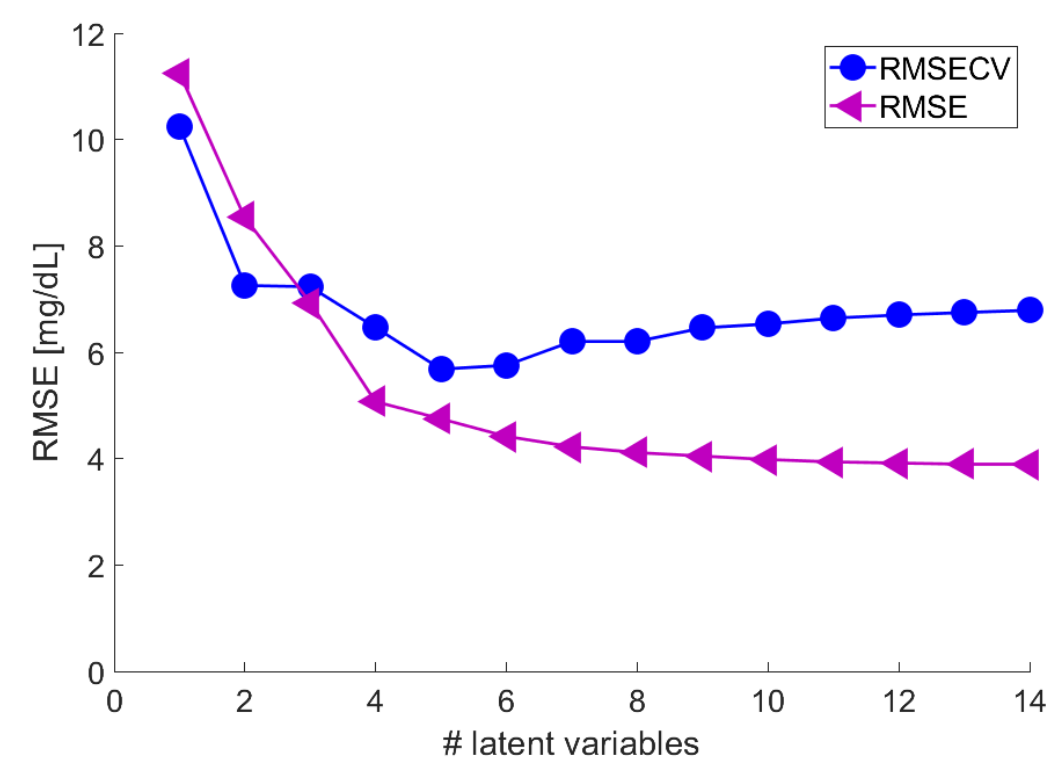

Figure 2.19.: RMSE with respect to the number of latent variables calculated by PLS with independent validation set (RMSECV, blue circles) and with validation within the training set (RMSE, violet triangles). An effect of over-fitting with increasing number of latent variables is observable. 


\subsection{Diffusion}

As the sensor's glucose exchange with the surrounding is based on passive molecular exchange between the fluidic reservoir and the measurement chamber via the holes in the silicon wafer, this phenomenon of diffusion has to be further investigated. This is shown in the following with the focus on diffusion time with respect to the sensor's geometry.

sec:theory-of-diffusion

\subsubsection{Theory of diffusion}

Fick's first law of diffusion describes the flux $J_{x}$ of a substance in direction $x$ in the presence of a concentration gradient $\frac{\partial c}{\partial x}$

$$
J_{x}=A D \frac{\partial c}{\partial x}
$$

with $D$ being the diffusion coefficient and $A$ being the area of the diffusion interface. The time dependent change in concentration $\partial c / \partial t$ in free solutions is described by Fick's second law as shown in Eq. 2.27 with $c(x, t)$ being the concentration at time $t$ and position $x$.

$$
\frac{\partial c(x, t)}{\partial t}=D A \frac{\partial^{2} c(x, t)}{\partial x^{2}}
$$

In the following an appraisal of the diffusion properties for the case of two volumina with different glucose concentrations being separated by a semipermeable membrane is given according to Adam et al. [69]. It is based on the assumption that the two volumina are distinct. The time dependent concentration difference between both volumina is then

$$
\Delta c=c^{I I}-c^{I}
$$

with $c^{I}$ being the concentration in volume 1 and $c^{I I}$ being the concentration in volume 2. Taking into account that the exchange rate $d n^{I} / d t$ on one side of the membrane has to lead to a negative change of the same amount on the other side of the membrane and also inserting Fick's first law of diffusion (Eq. 2.26) with $d$ being the thickness of the membrane i.e. the diffusion length it follows

$$
\frac{d n^{I}}{d t}=V^{I} \frac{d c^{I}}{d t}=D A \frac{\Delta c}{d}==-\frac{d n^{I I}}{d t}=-V^{I I} \frac{d c^{I I}}{d t}=-D A \frac{\Delta c}{d} .
$$

The change rate in concentration change can then be acquired according to

$$
\frac{d \Delta c}{d t}=\frac{d c^{I I}-d c^{I}}{d t}=-\frac{D A}{d}\left(\frac{1}{V^{I}}+\frac{1}{V^{I I}}\right) \Delta c .
$$

Integration of this results leads to a time dependent formula of the absolute concentration change over the surface 


$$
\Delta c=(\Delta c)_{0} e^{-\frac{D A}{d}\left(\frac{1}{V^{I}}+\frac{1}{V^{I I}}\right) t}
$$

and, correspondingly, to a behavior of the concentration in the measurement chamber according to

$$
c^{I}=c^{I I}\left(1-e^{-\frac{D A}{d}\left(\frac{1}{V^{I}}+\frac{1}{V^{I I}}\right) t}\right) .
$$

We introduce a time constant $\tau$ to characterize the diffusion process according to

$$
c^{I}=c^{I I}\left(1-e^{-\frac{t}{\tau}}\right) \text { with } \tau=\frac{d}{D A\left(\frac{1}{V^{I}}+\frac{1}{V^{I I}}\right)} .
$$

The diffusion coefficient $D$ of a substance is a quantity that depends on the temperature, viscosity of the fluid and the size of the particles [70]. It is commonly described by the Stokes-Einstein equation (for diffusion of spherical particles in a liquid with low Reynolds number):

$$
D=\frac{k_{B} T}{6 \pi \eta R_{s}}
$$

with $k_{B}$ being the Boltzmann constant, $T$ being the temperature, $\eta$ being the dynamic viscosity and $R_{s}$ being the Stokes radius of the particles. The Stokes radius can be approximated by the molecular radius $R_{0}$ [71] using

$$
R_{0}=\left[\frac{3 M}{4 \pi \rho N_{A}}\right]^{1 / 3}
$$

with $M$ being the molecular weight, $\rho$ being the density of the molecule and $N_{A}$ being Avogadro's number.

\subsubsection{Simulation}

For the description of more complex systems than the above approximated case of two volumina separated by a semipermeable membrane, as e.g. it is the case with conical holes in the silicon wafer, it is common to use so-called finite element simulation tools. These methods assign the geometry of interest to a grid of small finite elements (volumina in our case) and assumes conservation of mass, momentum and energy in every point in the grid. These quantities are intrinsically coupled by a nonlinear coupled differential equation system, the so called Navier-Stokes equation. As this system is hardly solvable analytically, it is numerically solved by the simulation at each point in the grid under given boundary conditions. In this thesis a finite volume simulation tool called Flow3D (Flow Science, Inc., Santa Fe, USA) is used for the simulation of diffusion properties of glucose through the holes in the silicon wafer. Figure 2.20 gives a side view of the hole geometry with the chosen grid indicated in black. A silicon wafer (thickness $200 \mu \mathrm{m}$ ) with a hole (upper diameter $10 \mu \mathrm{m}$ ) and a 
$12 \mu \mathrm{m}$ liquid layer on top (measurement chamber) and a large fluidic reservoir below the wafer is implemented. The top boundary (above the measurement chamber) is assumed to be an impermeable wall, whereas the sides are assumed to be symmetry boundary layers, meaning that the simulation assumes no velocities normal to the layer. For a reduction in simulation time only the smallest unit cell containing one hole and the half distance to the next hole is simulated (continuation of the wafer is shown in shaded color). We assumed constant pressure and no convection present in the system. The simulation's starting conditions at time point $t=0$ are the whole volume filled with liquid (water) and the volume in the measurement chamber and the hole filled with water (glucose concentration 0) and the fluidic reservoir filled with aqueous glucose solution (glucose concentration $100 \mathrm{mg} / \mathrm{dL}$ ). As an additional boundary condition the inflow of at the boundaries surrounding the fluidic reservoir is set to the same glucose concentration $(100 \mathrm{mg} / \mathrm{dL})$ in order to imitate a infinitely large fluidic reservoir (with no concentration change over time). By carrying out the simulation, the concentration inside the measurement chamber can be monitored over time in order to fit an exponential curve of the form shown in Equation 2.33 and to calculate the diffusion time constant $\tau$ for different hole geometries.

Figure 2.21 A shows the simulated diffusion time with respect to the hole diameter and Figure 2.21 B with respect to the silicon wafer thickness. $\tau$ shows a behavior that is inverse proportional to diffusion area and linear proportional to the diffusion length. Thus the simulation result shows the same behavior as expected from the appraisal in free solution shown in Sec. 2.2.1.

Dependence of diffusion time on the opening angle does not show a large change at angles between $5^{\circ}$ to $30^{\circ}$ (a decrease of about 20\%) compared to the dependence on hole diameter and silicon layer thickness.

\subsubsection{Measurement parameters}

Experimental diffusion measurements are done by changing the glucose concentration in the fluidic reservoir (with a pumping velocity of $30 \mathrm{rpmin}$ ) from 0 to $500 \mathrm{mg} / \mathrm{dL}$ (and vice versa) and simultaneously measuring the glucose absorption in the measurement chamber. This optical measurement is done at a fixed wavenumber of $1045 \mathrm{~cm}^{-1}$ in the glucose absorption region (for glucose, $500 \mathrm{mg} / \mathrm{dL}$ and maltodextrin, $500 \mathrm{mg} / \mathrm{dL}$ ) and a wavenumber of $1548 \mathrm{~cm}^{-1}$ for albumin $1000 \mathrm{mg} / \mathrm{dL}$. Diffusion measurements of the substance diffusing into the chamber and of the substance diffusing out of the chamber (by puming water into the fluidic reservoir) are done in alternation. In addition, also diffusion measurements with an additional ultrafiltration membrane (details see Sec. 2.3) in the opto-fluidic interface are done with glucose and maltodextrin solutions.

For the investigation of the temperature dependence of diffusion time the optofluidic interface was hold at a particular temperature using the temperature PID controller described in Sec. 2.1.2. 


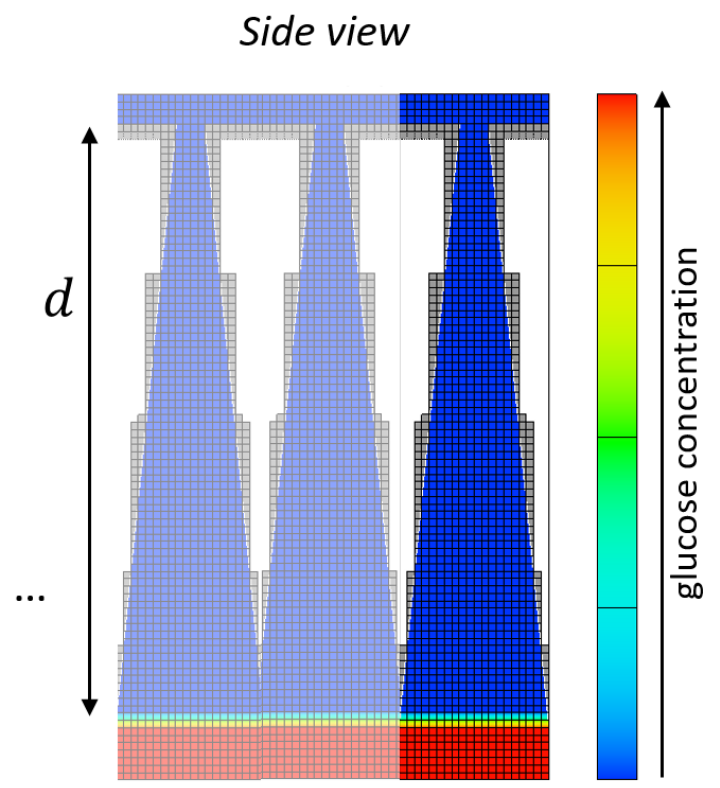

Figure 2.20.: Schematic of the implemented 3D geometry used for the finite element simulation of diffusion into the sensor. The side view shows the cut through the holes that are conically drilled into the silicon wafer. The blue and red area indicate areas with liquid. Below the silicon wafer a fluidic reservoir (shown in red) is implemented, at time point 0 the glucose concentration in the reservoir is $100 \mathrm{mg} / \mathrm{dL}$ (indicated in red) and in the rest of the volume it is $0 \mathrm{mg} / \mathrm{dL}$. During the simulation the glucose diffusion through the holes into the measurement chamber is monitored over time. The black mesh indicates the finite volume elements that are used for discretized calculation. The size of the single mesh elements is chosen by a compromise between being small enough to realistically reproduce the real geometry and large enough to stay in a feasible calculation time. A mesh size of $2 \mu \mathrm{m}$ is used in all dimension. 


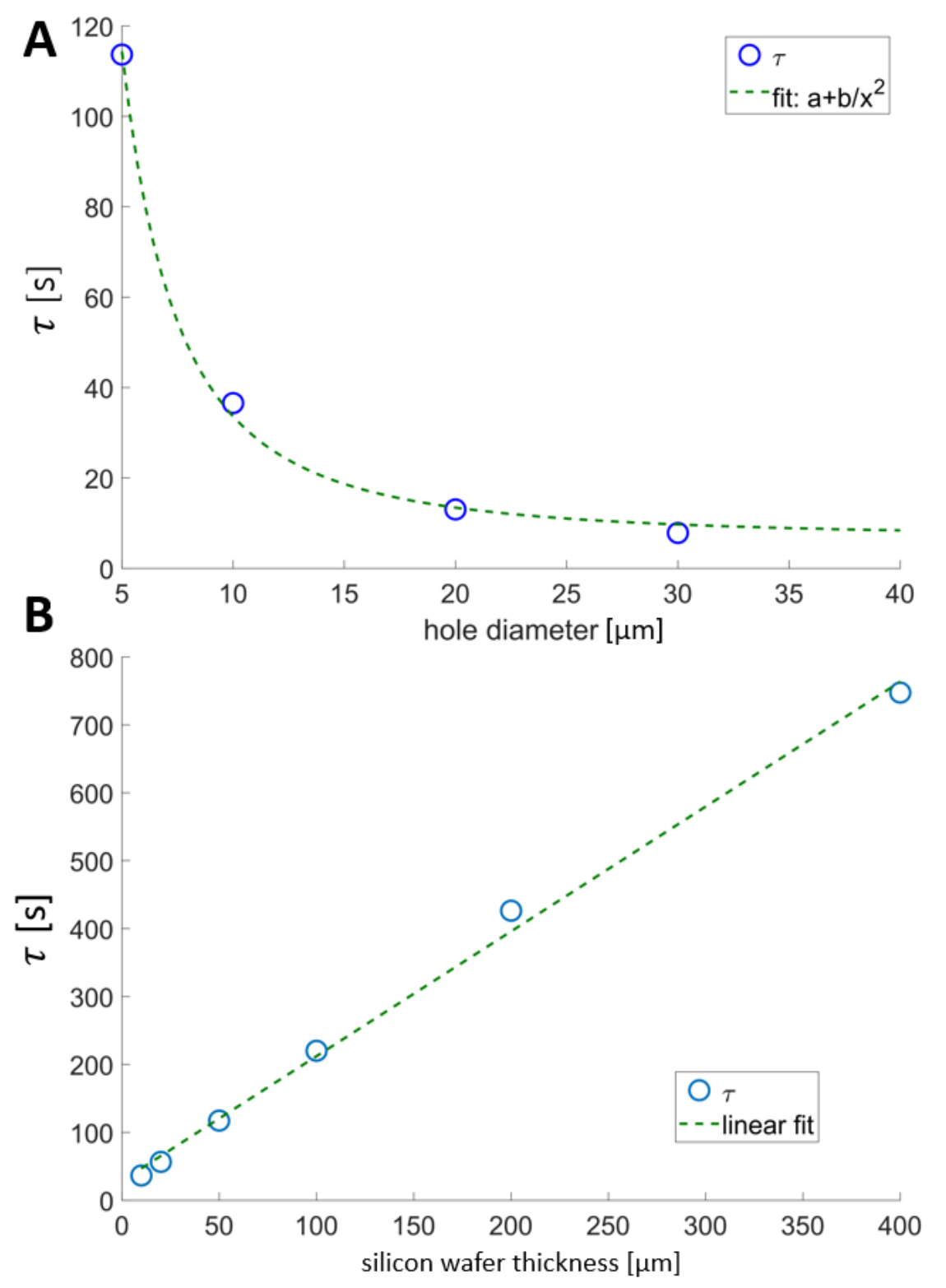

Figure 2.21.: A: Dependence of glucose diffusion time on the diameter of the hole. The curve shows an inverse quadratic dependency as indicated by the dashed line. B: Dependence of diffusion time on the thickness of the silicon wafer. Here a linear dependence (dashed line) is observable. 


\subsection{Long-term measurements}

For the investigation of the long-term sensor stability the opto-fluidic setup is left in its configuration for a time period of 42 days and periodically characterized by measurements. To test the performance under the influence of proteins, the measurement solutions contained physiological concentrations of albumin. Further the opto-fluidic interface is modified by adding an additional regenerated cellulose membrane (15004, Reichelt Chemietechnik, Germany, see Sec. 2.1.2) with a nominal weight cutoff of $25 \mathrm{kDa}$. This is done in order to let only small molecules such as glucose (molecular weight $0.18 \mathrm{kDa}$ ) pass while blocking large molecules such as albumin (molecular weight $66 \mathrm{kDa}$ ). A enrichment of proteins in the measurement chamber would lead in long-term (among others) to a change in reflectivity of the gold layer and thus a change in the sensitivity of the sensor. To probe this filtration effect the measurement solutions are aqueous solutions of $500 \mathrm{mg} / \mathrm{dL}$ glucose and $1000 \mathrm{mg} / \mathrm{dL}$ albumin as well as a solution of $1000 \mathrm{mg} / \mathrm{dL}$ albumin for referencing. One measurement cycle is done by first measuring the diffusion time of glucose in the presence of albumin by a fixed-frequency measurement at $1045 \mathrm{~cm}^{-1}$ (as described in 2.2.3). After an equilibrium in glucose concentration is reached inside the sensor, spectral measurements are done by 4 sweep scans of QCLG $\left(900 \mathrm{~cm}^{-1}\right.$ to $\left.1200 \mathrm{~cm}^{-1}\right)$ followed by 4 sweep scans of QCLP $\left(1500 \mathrm{~cm}^{-1}\right.$ to $\left.1750 \mathrm{~cm}^{-1}\right)$. Afterwards the same procedure is done with the solution of $1000 \mathrm{mg} / \mathrm{dL}$ albumin only. 

3. Experiments and results 


\section{Experiments and results}

In this chapter the sensor's in vitro performance is evaluated with respect to the following points: its glucose sensitivity and selectivity in pure aqueous glucose solutions and under the impact of other biologically relevant substances present in solution; the impact of temperature changes on the spectral signal and the glucose prediction accuracy; its response time to a concentration change in the sensor's surrounding and its long-term performance and stability.

\subsection{Spectroscopic quantification of glucose}

An overview of the spectral performance of the setup will be given in this section. The glucose prediction sensitivity in pure glucose solutions under different cross validation procedures will be shown first (3.1.1), followed by the spectral impact of other substances present in solution, and the impact of temperature changes (3.1.2).

\subsubsection{Measurements of pure glucose solutions}

The measurements shown in the following are based on 5 measurement series taken within a time period of 3 months. Each measurement series contains 5 to 8 measurement cycles with 6 to 10 different concentrations in each cycle (242 measurements in total, 13 different glucose concentrations between $0 \mathrm{mg} / \mathrm{dL}$ to $1000 \mathrm{mg} / \mathrm{dL}$ ). In each cycle, a series of randomized concentrations is measured. Every third measurement consists of an additional water measurement which is used as reference in the calculation of the transflectance. This ensures that there is a reference measurement either before or after each glucose measurement.

The transflectance spectra of different glucose concentrations are shown in Figure 3.1 B. For comparison, absorbance spectra (taken by Müller [17] using a fiber-based glucose sensor setup) are shown in Figure $3.1 \mathrm{~A}$. The spectra roughly resemble each other and the absorption features typical for glucose around $1035 \mathrm{~cm}^{-1}, 1080 \mathrm{~cm}^{-1}$ and $1155 \mathrm{~cm}^{-1}$ are observable in either measurement technique. However some spectral regions show higher absolute transflectance than in transmission geometry, caused by the nonlinear transflectance with respect to wavenumber (as shown in 2.1.4). This effect is, for example, observable around $980 \mathrm{~cm}^{-1}$ and $1155 \mathrm{~cm}^{-1}$.

To set a baseline, the glucose sensitivity is first evaluated at each wavenumber, i.e. a univariate linear regression of the transflectance with respect to concentration is performed at every wavenumber. The $\mathrm{RMSECV}_{\text {cycle }}$ for every respective wavenumber is shown in Figure 3.2. A minimum RMSECV $\mathrm{V}_{\text {cycle }}$ of $10.7 \mathrm{mg} / \mathrm{dL}$ is reached at a wavenumber of $1044 \mathrm{~cm}^{-1}$. This value is already in a satisfying order of magnitude for a sufficient glucose determination.

When using the spectral data instead of the data from a single wavenumber, much more information about the glucose content can be obtained. Multivariate calibration with a PLS model results in a RMSECV below $10 \mathrm{mg} / \mathrm{dL}$ as will be shown in the following. The measurement series are investigated in terms of intra-dayvariance (variance from cycle to cycle within one day), inter-day-variance (variance 


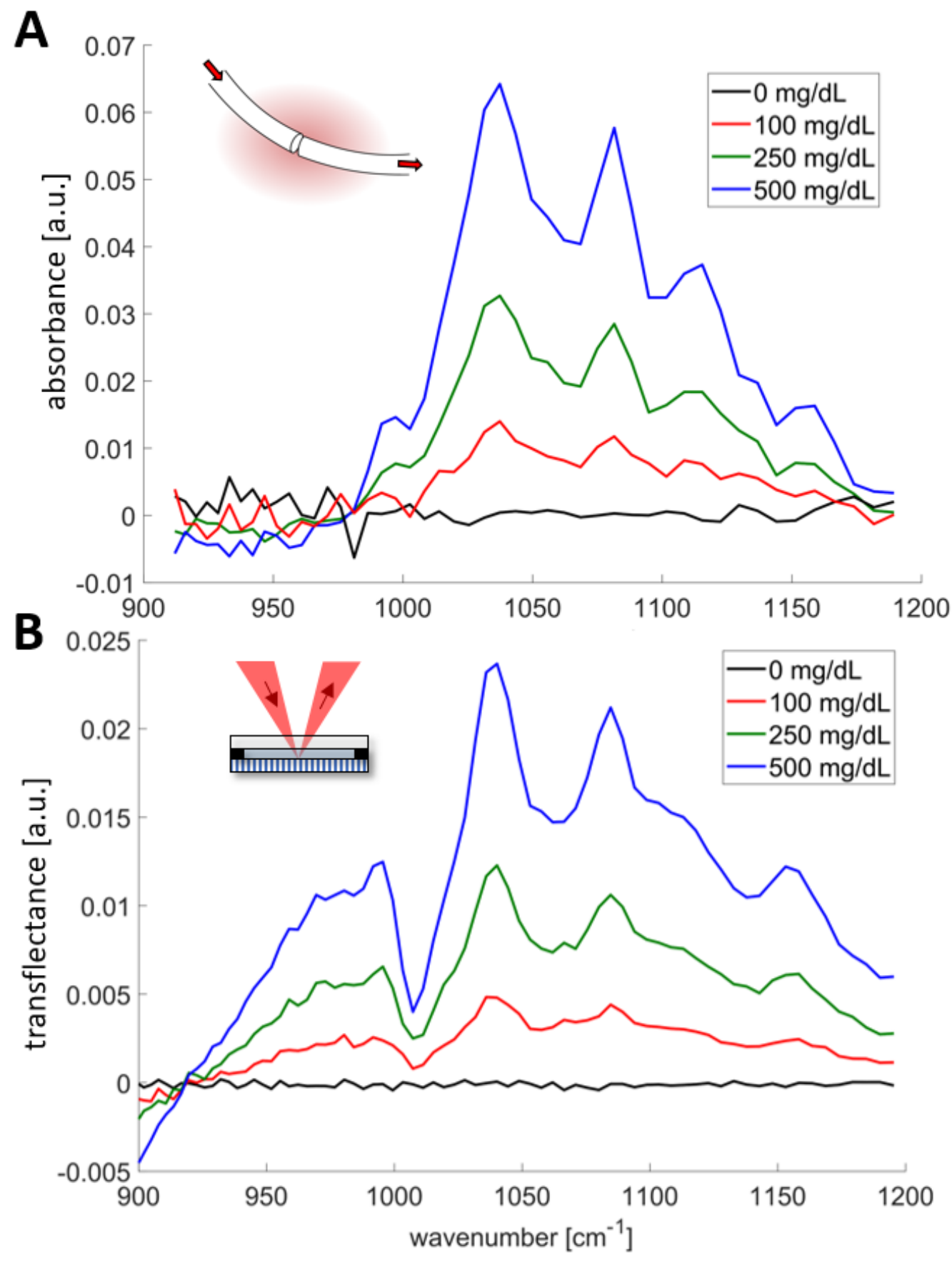

Figure 3.1.: Glucose (decadic) absorbance and transflectance spectra of different concentrations. A: Spectra acquired in transmission geometry using a fiber-based sensor [16, 17], B: Spectra acquired in transflection geometry. Figure adapted from Isensee et al. [25]. 


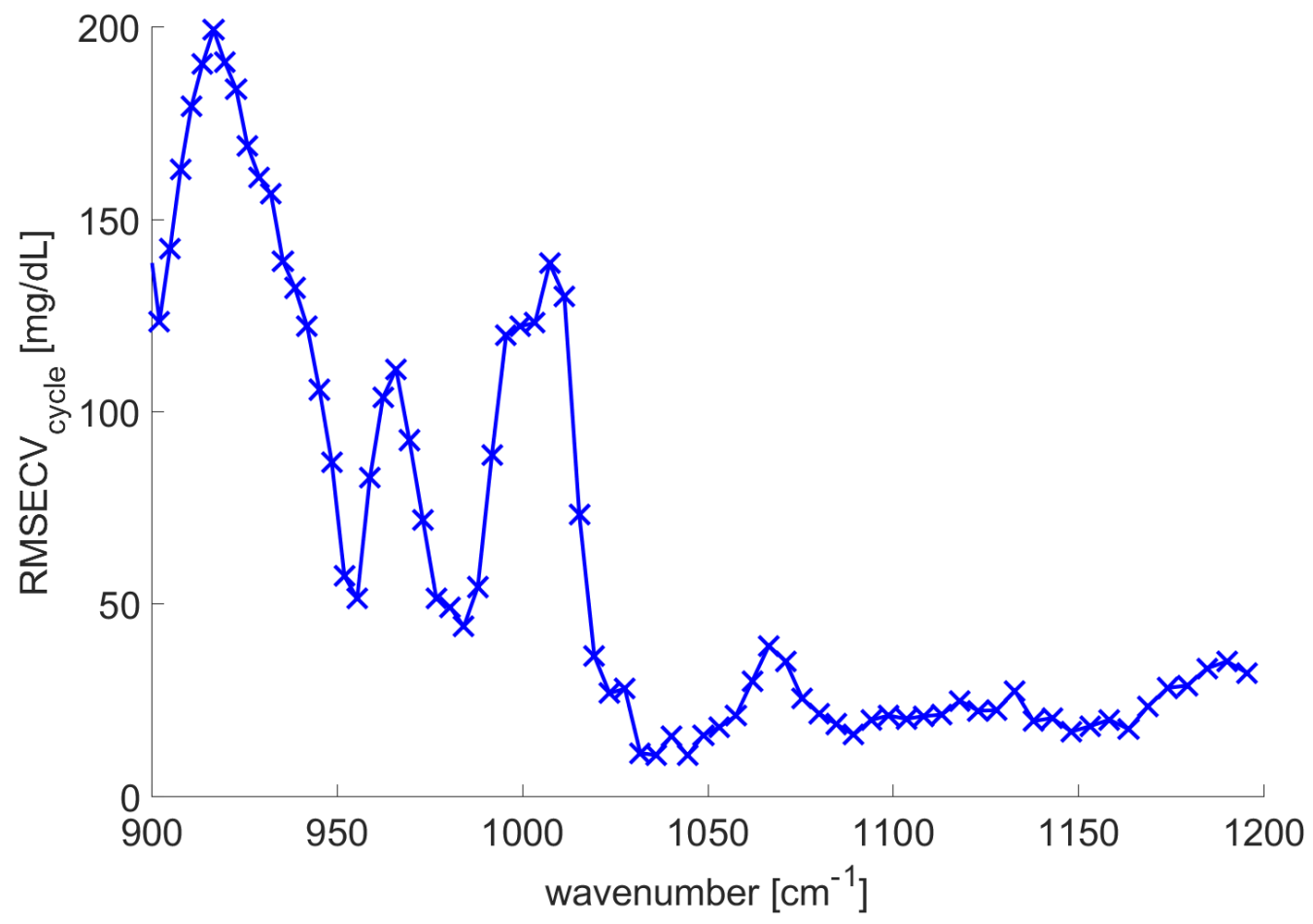

Figure 3.2.: Univariate RMSECV $\mathrm{V}_{\text {cycle }}$ on each wavenumber in pure glucose solutions.

from cycle to cycle within different days, 'leave-on-cycle-out') and day-to-day variance (variance between one day and another day, 'leave-one-day-out'). The result of a multivariate analysis of the spectra using PLS is shown in Table 3.1. It is calculated using referenced transflectance spectra (referenced on respective adjacent water measurement). For comparison, the respective result calculated on the not referenced intensity spectra is shown as well. In order to obtain the best RMSECV, the PLS is optimized with respect to the number of latent variables (see Figure 3.3).

Investigation of the data set containing measurements of 5 days within a time period of 3 months leads to a (day-to-day) $\mathrm{RMSECV}_{\text {day }}$ as low as $7.5 \mathrm{mg} / \mathrm{dL}$ in comparison to a (inter-day) $\mathrm{RMSECV}_{\text {cycle }}$ of $6.0 \mathrm{mg} / \mathrm{dL}$ on the same dataset. This shows that the inter-day variance is in the same range as the day-to-day variance. Further, as the sensor was disassembled in between the different measurement-days (because the QCL-setup was needed for other experiments), basically no acquisition of novel training data (for calibration of the PLS) at the exact same setup or day is needed for a suffcient RMSECV in pure glucose solutions. However, a comparison with the results obtained on the non-referenced signals shows that the use of a water reference is essential (the role of the water reference will be discussed in detail in Section 3.1.2).

The most sensitive RMSECV $\mathrm{V}_{\text {cycle }}$ of $3.1 \mathrm{mg} / \mathrm{dL}$ is achieved within a measurement series taken on just a single day. This accuracy within one single measurement series is expected as the data set does not contain variances between different days 
Table 3.1.: Glucose prediction by PLS analysis in pure aqueous solutions at constant temperature

\begin{tabular}{|c|c|c|c|c|}
\hline \multirow[t]{2}{*}{ Cross-validation } & \multirow{2}{*}{$\begin{array}{l}\text { Measurement } \\
\text { within }\end{array}$} & \multirow{2}{*}{$\begin{array}{l}\text { \# Latent } \\
\text { variables }\end{array}$} & \multicolumn{2}{|c|}{$\mathrm{RMSECV}[\mathrm{mg} / \mathrm{dL}]$} \\
\hline & & & with reference & without reference \\
\hline leave-one-cycle-out & intra-day & 3 & 3.1 & 10.0 \\
\hline leave-one-cycle-out & $\begin{array}{l}5 \text { days within } 3 \\
\text { months }\end{array}$ & 4 & 6.0 & 91.5 \\
\hline leave-one-day-out & $\begin{array}{l}5 \text { days within } 3 \\
\text { months }\end{array}$ & 4 & 7.5 & 931.9 \\
\hline
\end{tabular}

or setups .

Figure 3.4 shows the predicted values in a Parkes error grid with respect to the evaluation method. One can see that in terms of clinical glucose monitoring performance all predicted values lay in the medically ideal region (region A). Figure 3.5 shows the absolute deviation from the real concentration. It seems that the prediction of glucose concentrations of $0 \mathrm{mg} / \mathrm{dL}$ leads to a high absolute error up to $40 \mathrm{mg} / \mathrm{dL}$. This is presumably caused by the fact that the used reference measurement at $0 \mathrm{mg} / \mathrm{dL}$ glucose (i.e. the next water measurement) is not 1 but 3 measurements apart (based on the measurement scheme of one water measurement every 3 measurements). However, this issue can be neglected as such low concentrations lay outside the physiologically relevant region.

\subsubsection{Environmental impacts}

In this section the effect of environmental impacts on the sensor's in vitro performance will be addressed. These are the presence of other substances in solution, namely saccharides and proteins and temperature changes of the sensor.

\section{Impact of other saccharides}

With the aim for a continuous glucose monitoring implant in vivo in mind, the sensors selectivity for glucose has to be guaranteed as there are many other substances besides glucose present in the interstitial fluid. Based on their intrinsic molecular structure all other substances should be distinguishable from glucose by their vibrational spectrum in the mid infrared. However, molecules that are very similar to glucose with regards to their structure have very similar mid-IR absorption properties and, thus, are very hard to distinguish by their spectrum. To investigate an extreme case of similarity between molecules, we investigate the glucose selectivity among high concentrations $(0 \mathrm{mg} / \mathrm{dL}$ to $500 \mathrm{mg} / \mathrm{dL}$ ) of the saccharides maltose, lactose, galactose and fructose. This can also be considered as an upper limit in terms of concentrations as typical concentrations of these molecules in the interstitial fluid are much lower in the range of $0 \mathrm{mg} / \mathrm{dL}$ to $18 \mathrm{mg} / \mathrm{dL}$ [13]. 6 measurement series 


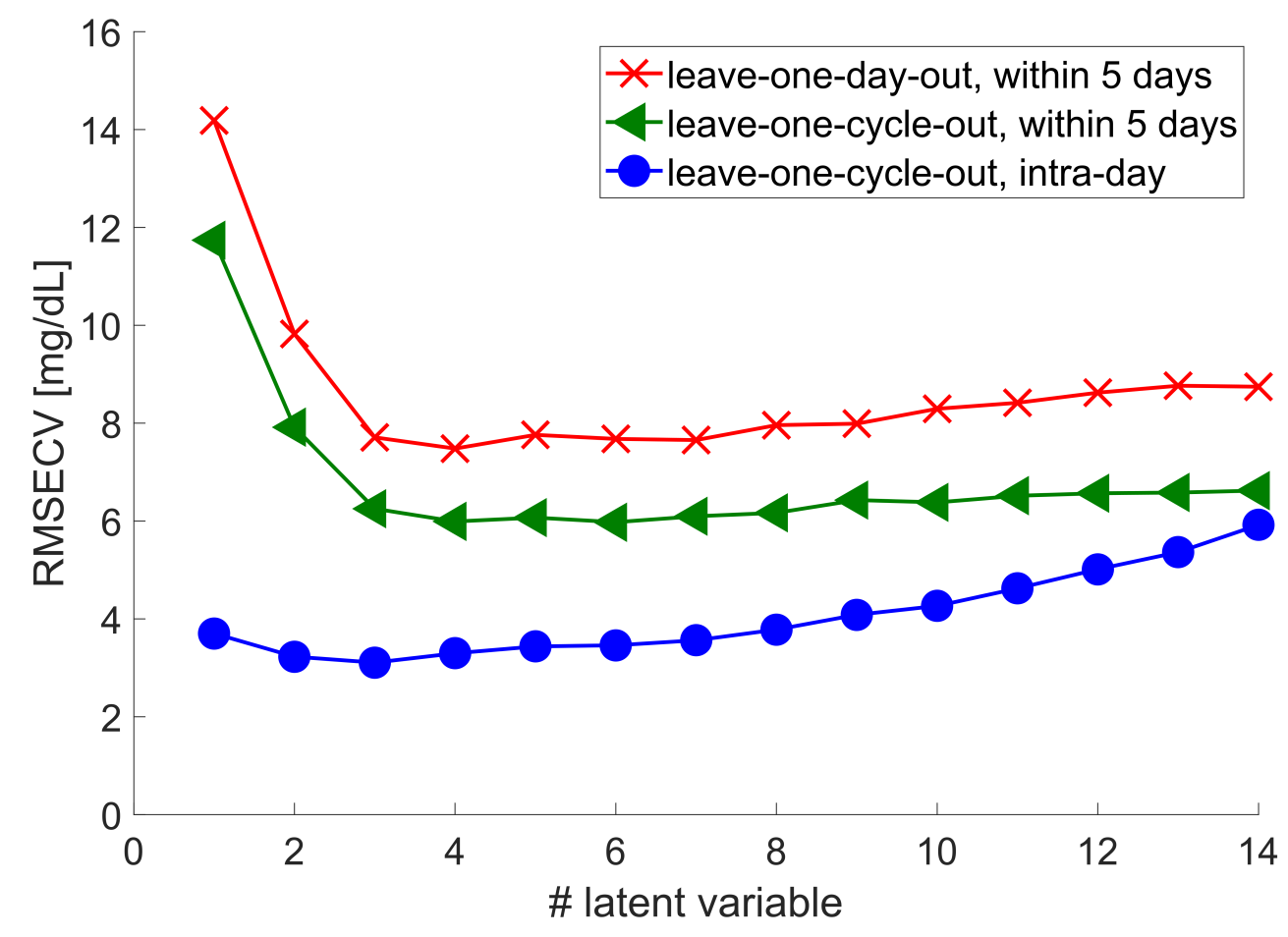

Figure 3.3.: Overview on the impact of chosen number of latent variables on the RMSECV of glucose concentration. The different symbols indicate different cross-validation methods: leave-one-cycle-out (blue circles), leave-onecycle-out within a timespan of 5 days (green triangles) and leave-oneday-out within a timespan of 5 days (red crosses). 


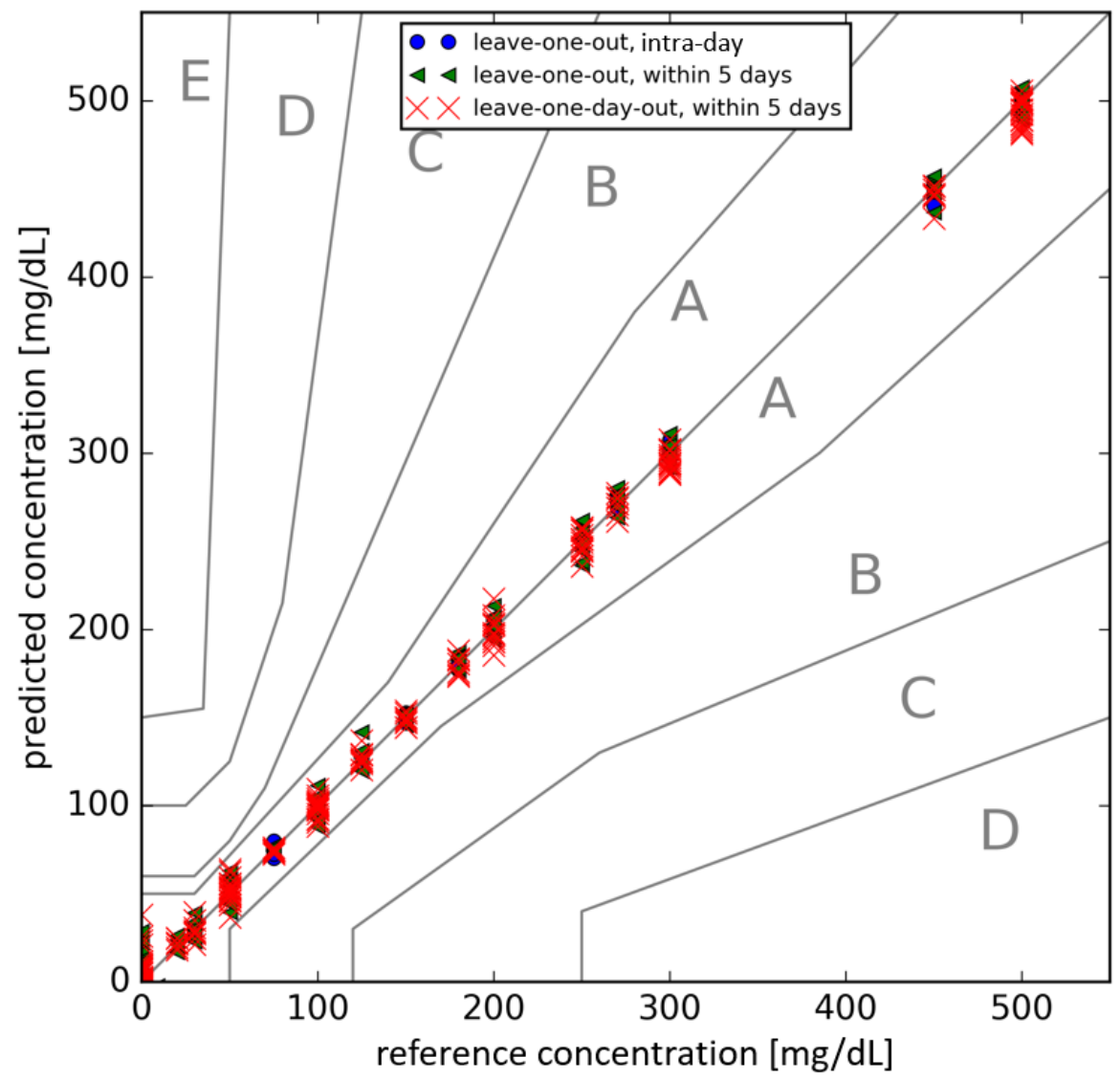

Figure 3.4.: Predicted glucose concentrations in pure glucose solutions shown in a Parkes error grid. The different symbols indicate different crossvalidation methods for the characterization of different variances: leaveone-cycle-out (intra day) with 2 latent variables in the PLS calibration (blue circles), leave-one-cycle-out within a timespan of 5 days (inter-day) with 4 latent variables (green triangles) and leave-one-day-out within a timespan of 5 days (day-to-day) with 4 latent variables (red crosses). Figure adapted from Isensee et al. [25]. 


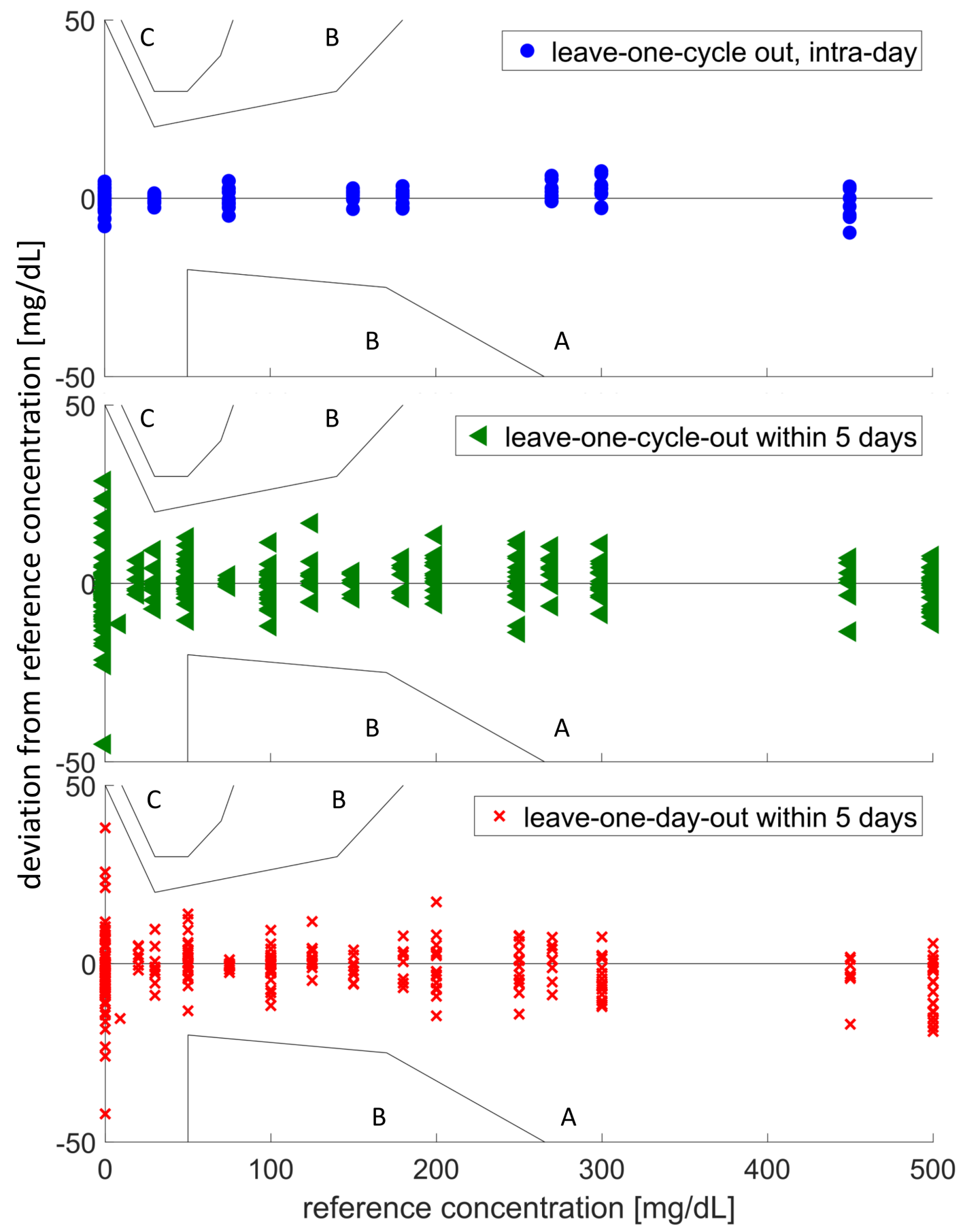

Figure 3.5.: Absolute deviation of predicted glucose concentration from the reference value. The different symbols indicate different cross-validation methods for the characterization of different variances: leave-one-cycle-out (intra day) with 2 latent variables in the PLS calibration (blue circles), leave-one-cycle-out within a timespan of 5 days (inter-day) with 4 latent variables (green triangles) and leave-one-day-out within a timespan of 5 days (day-to-day) with 4 latent variables (red crosses). 
Table 3.2.: Glucose prediction within a mixture of other saccharides as obtained by PLS analysis (leave-one-cycle-out crossvalidation, 7 latent variables)

\begin{tabular}{lcl}
\hline & Concentrations $[\mathrm{mg} / \mathrm{dL}]$ & RMSECV $_{\text {cycle }}[\mathrm{mg} / \mathrm{dL}]$ \\
\hline glucose & $0-600$ & \\
maltose & $0-500$ & 20.3 \\
fructose & $0-500$ & \\
galactose & $0-500$ & \\
lactose & $0-500$ & \\
\hline glucose & $0-600$ & 13.6 \\
fructose & $0-500$ & \\
galactose & $0-500$ & \\
lactose & $0-500$ & 11.9 \\
\hline glucose & $0-600$ & \\
fructose & $0-500$ & \\
galactose & $0-500$ & \\
\hline
\end{tabular}

containing 8 measurement cycles with 8 different solutions each are carried out (512 measurements in total), whereof the first 4 measurement series contained random mixtures of $50 \mathrm{mg} / \mathrm{dL}, 100 \mathrm{mg} / \mathrm{dL}, 200 \mathrm{mg} / \mathrm{dL}$ and $600 \mathrm{mg} / \mathrm{dL}$ glucose in a mixture with 0 or $100 \mathrm{mg} / \mathrm{dL}$ of fructose, galactose and lactose as well as 0 or $500 \mathrm{mg} / \mathrm{dL}$ of maltose and the last 2 measurement series contains pure solutions of $0 \mathrm{mg} / \mathrm{dL}$, $100 \mathrm{mg} / \mathrm{dL}, 250 \mathrm{mg} / \mathrm{dL}$ and $500 \mathrm{mg} / \mathrm{dL}$ of the individual saccharides.

The transflectance spectra of pure solutions of the individual saccharides referenced to an adjacent water measurement are shown in Figure 3.6. Again, a comparison between absorbance spectra (measured in transmission geometry) and transflectance spectra is shown. As already experienced in the comparison of pure glucose spectra, the transflectance shows spectral regions with higher peaks than measured in transmission. As expected from their molecular structure the spectra of the different saccharides look quite similar. However there are small differences in the peak ratios between the individual saccharides. When measuring at only a single wavenumber, other saccharides lead to cross correlation with glucose and a selectivity for glucose cannot be guaranteed as already seen by Vrancî́ [22] and Müller [17]. However, this problem becomes feasible when multivariate data analysis and thus the information of the whole spectrum is used. The resulting RMSECV cycle $_{\text {ch }}$ values for glucose are listed in Table 3.2 for a mixture of all saccharides, all saccharides with maltose excluded and monosaccharides only. As shown in Figure 3.7 more numbers of latent variables are needed for an optimum RMSECV compared to measurements in pure solutions. This is expected as there are more independent factors impacting the spectrum compared to pure glucose solutions where the only varied component is the glucose concentration. The worst glucose selectivity of RMSECV $_{\text {cycle }}=20.3 \mathrm{mg} / \mathrm{dL}$ is observed in a mixture of all saccharides investigated. 
Table 3.3.: Glucose prediction within mixtures with various albumin concentrations $(0-1000 \mathrm{mg} / \mathrm{dL})$ taken within one day

\begin{tabular}{lll}
\hline Referencing & Used QCL & RMSECV $_{\text {cycle }}[\mathrm{mg} / \mathrm{dL}]$ \\
\hline adjacent water measurement & QCLG \& QCLP & 9.1 \\
no referencing & QCLG \& QCLP & 9.4 \\
adjacent water measurement & QCLG & 9.4 \\
no referencing & QCLG & 9.8 \\
\hline
\end{tabular}

Due to the molecular structure of maltose, which is basically two glucose molecules attached to each other, this substance has the largest impact on the glucose prediction. Without maltose in solution a $\mathrm{RMSECV}_{\text {cycle }}$ of $13.6 \mathrm{mg} / \mathrm{dL}$ can be achieved. It is important to note that in an in vivo application disaccharides won't be an issue as they are already digested in the small intestine to monosaccharides and thus won't be present in the interstitial fluid [72-74]. In this condition a RMSECV ${ }_{\text {cycle }}$ as low as $11.9 \mathrm{mg} / \mathrm{dL}$ can be gained which is comparable to the RMSECV in pure glucose solutions $(6 \mathrm{mg} / \mathrm{dL})$. Figure 3.8 shows the result in a Parkes error grid with (top) and without (bottom) disaccharides present in the solution. The absolute deviation with respect to the reference concentration is shown in Fig. 3.9. The predictions in mixtures of all saccharides show some points in medically critical regions $\mathrm{C}$ and $\mathrm{B}$. However, in a mixture of monosaccharides only, which is the more realistic case in terms of in vivo application, all points except for one lay in region A. Again there is a large uncertainty for very small concentrations, but, again, this uncertainty is not decisive in terms of diabetes management.

\section{Impact of albumin}

In order to imitate an in vivo environment in an in vitro setup, the presence of proteins in the interstitial fluid needs to be kept in mind. The total amount of proteins in the ISF is reported to be about $20 \mathrm{~g} / \mathrm{L}$ whereof about $10 \mathrm{~g} / \mathrm{L}$ is albumin $[75,76]$. The transflectance spectrum of albumin measured with QCLG and QCLP is shown in Figure 3.10 B. For comparison an absorbance spectrum of albumin (dried film measured by FTIR spectroscopy by Haase [77]) is shown in Figure 3.10 A. Around $1650 \mathrm{~cm}^{-1}$ and $1545 \mathrm{~cm}^{-1}$ the amide I and amide II bands characteristic for the secondary structure of proteins are present. As it was the case for saccharides, the spectral transflectance is different to the absorbance measured in transmission. From a measurement done within mixtures of glucose $(0 \mathrm{mg} / \mathrm{dL}$ to $500 \mathrm{mg} / \mathrm{dL})$ and albumin $(0 \mathrm{mg} / \mathrm{dL}$ to $1000 \mathrm{mg} / \mathrm{dL})$ and pure spectra of the substances (16 different concentrations, 8 measurement series, 64 measurements in total, using adjacent water spectra as reference) a PLS calibration achieves a $R M S E C V_{\text {cycle }}$ for glucose below $10 \mathrm{mg} / \mathrm{dL}$ (see Table 3.3). 


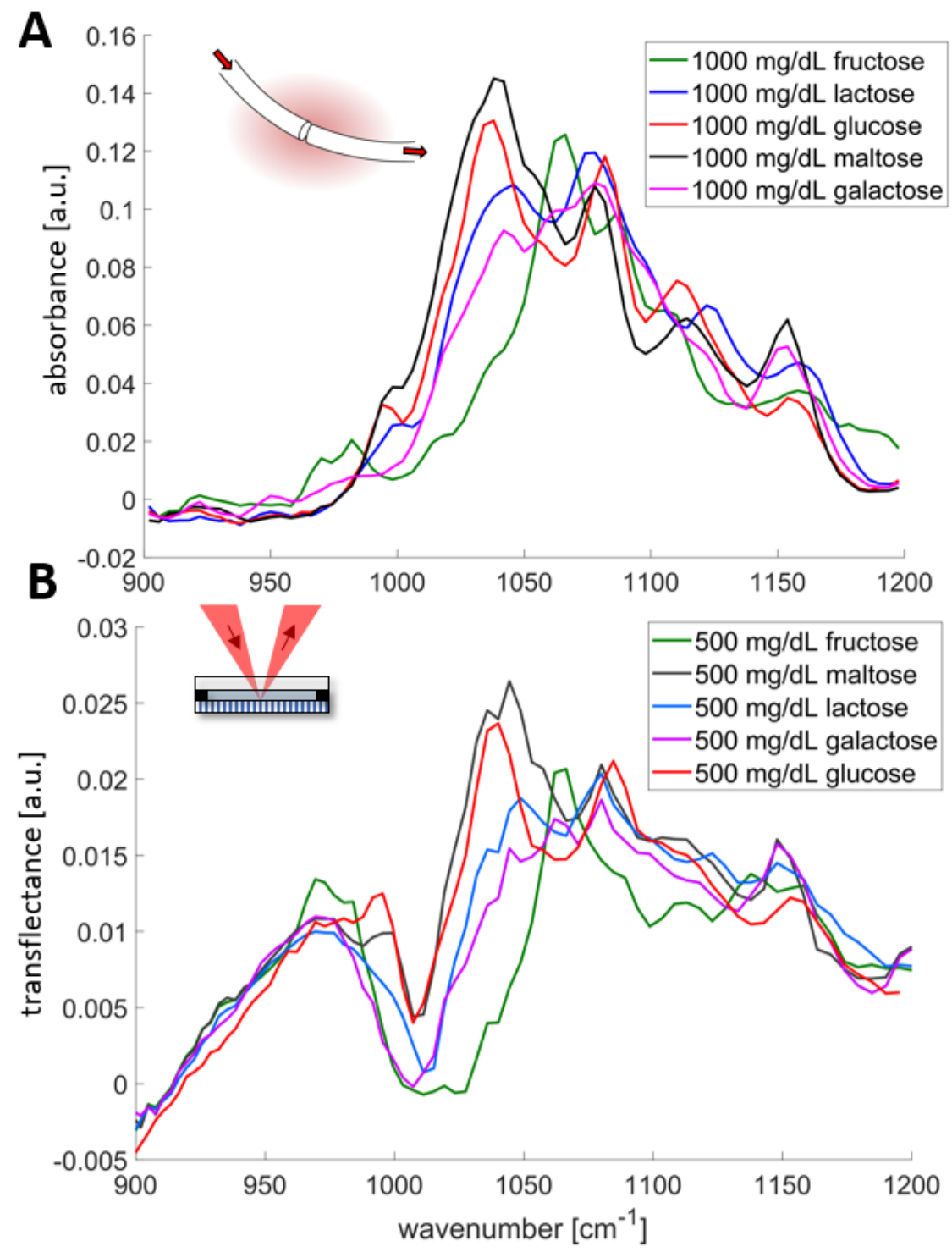

Figure 3.6.: (Decadic) absorbance and transflectance spectra of different saccharides. A: Spectra acquired in transmission geometry using a fiber-based sensor [16, 17], B: Spectra acquired in transflection geometry. Figures adapted from Isensee et al. [25]. 


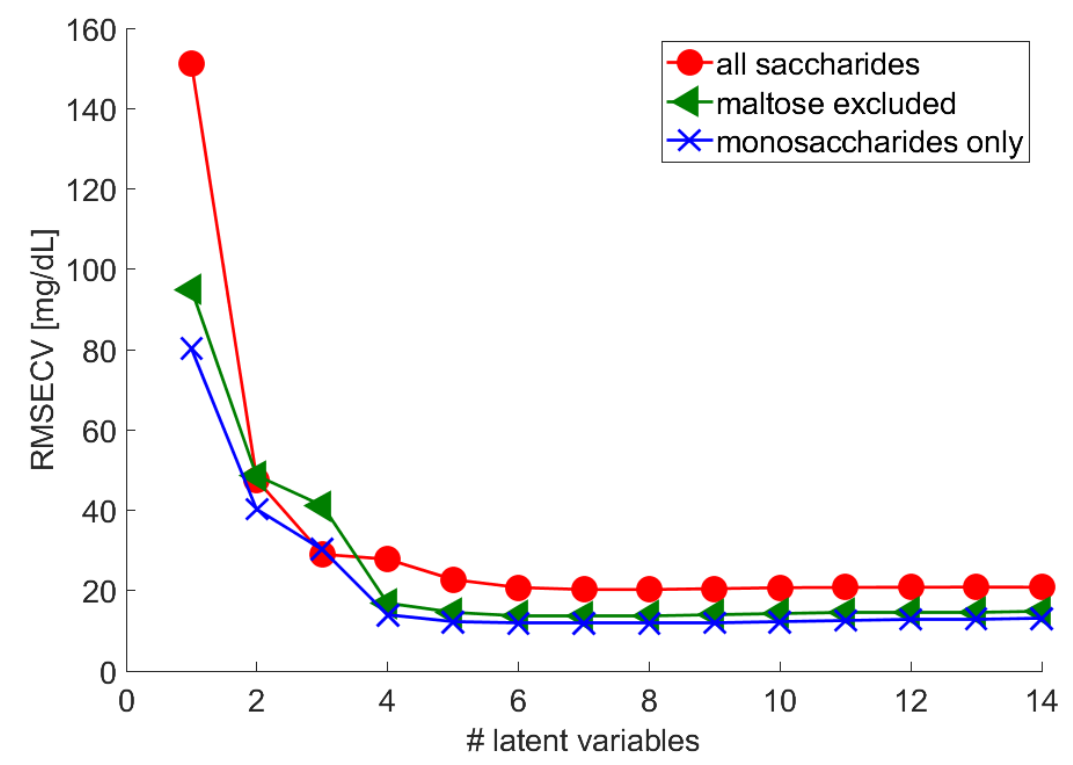

Figure 3.7.: RMSECV of glucose prediction with respect to the number of latent variables for a mixture of different mono-and-disaccharides (circles) and monosaccharides only (crosses).

\section{Role of water reference}

The sensor's performance without any water reference is analyzed on the dataset taken within a mixture of glucose and albumin. This is done as the water referencing may not adequately reflect the in vivo measurement scheme. A PLS analysis of the raw signal instead of the calculated transflectance (with water as a reference) is done and gives a RMSECV $V_{\text {cycle }}$ in the same range as the referenced dataset (see Table 3.3). This shows that within a measurement basically no water reference is required. However, this result has to be taken carefully because the measurements are all taken within a single day. As seen earlier in pure glucose solutions, the sensitivity of unreferenced signals can drastically drop among measurements taken at different days or different setups.

\section{Role of additional laser in another spectral region}

Further the question arises if a laser in a spectral region apart from the carbohydrate region is needed at all. To answer this, the impact of the additional laser (QCLP) on the glucose prediction (in the same dataset) is investigated by comparison of the result gained with both lasers and with QCLG only. Surprisingly, even though the highest transflectance of albumin is in the spectral region of QCLP, it does not add much information to the data set as the RMSECV $\mathrm{V}_{\text {cycle }}$ does not significantly change. The Parkes error grid (see Fig. 3.11) as well as the absolute deviation from the predicted value as shown in Fig. 3.12 indicate a sufficient glucose prediction accuracy for clinical use (nearly all values in region $\mathrm{A}$ ). 

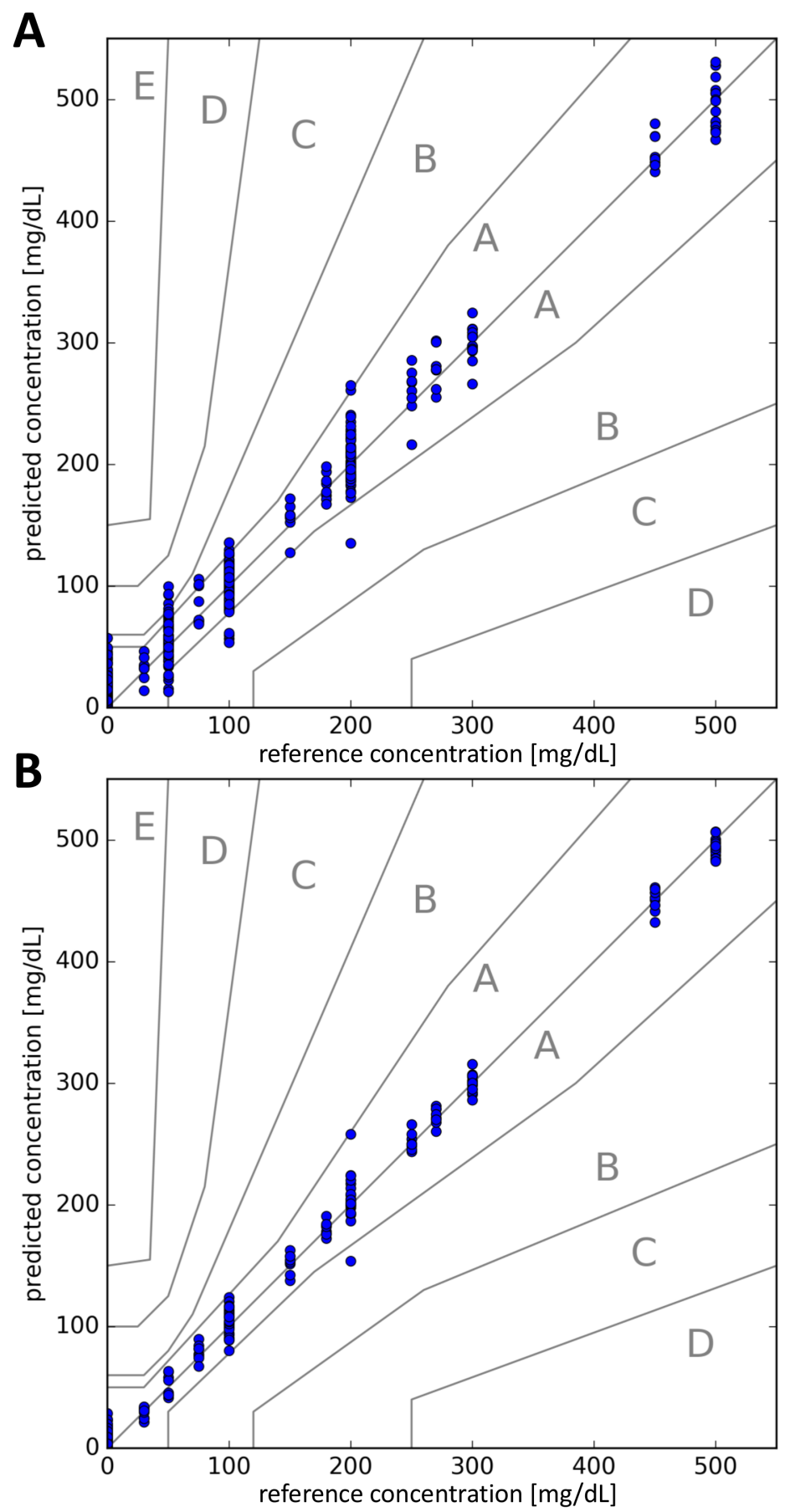

Figure 3.8.: Parkes error grid of the glucose concentrations within a mixture of other monosaccharides and disaccharides (A) and monosaccharides only (B) as predicted by PLS analysis (leave-one-cycle-out crossvalidation, 7 latent variables). Figures adapted from Isensee et al. [25]. 


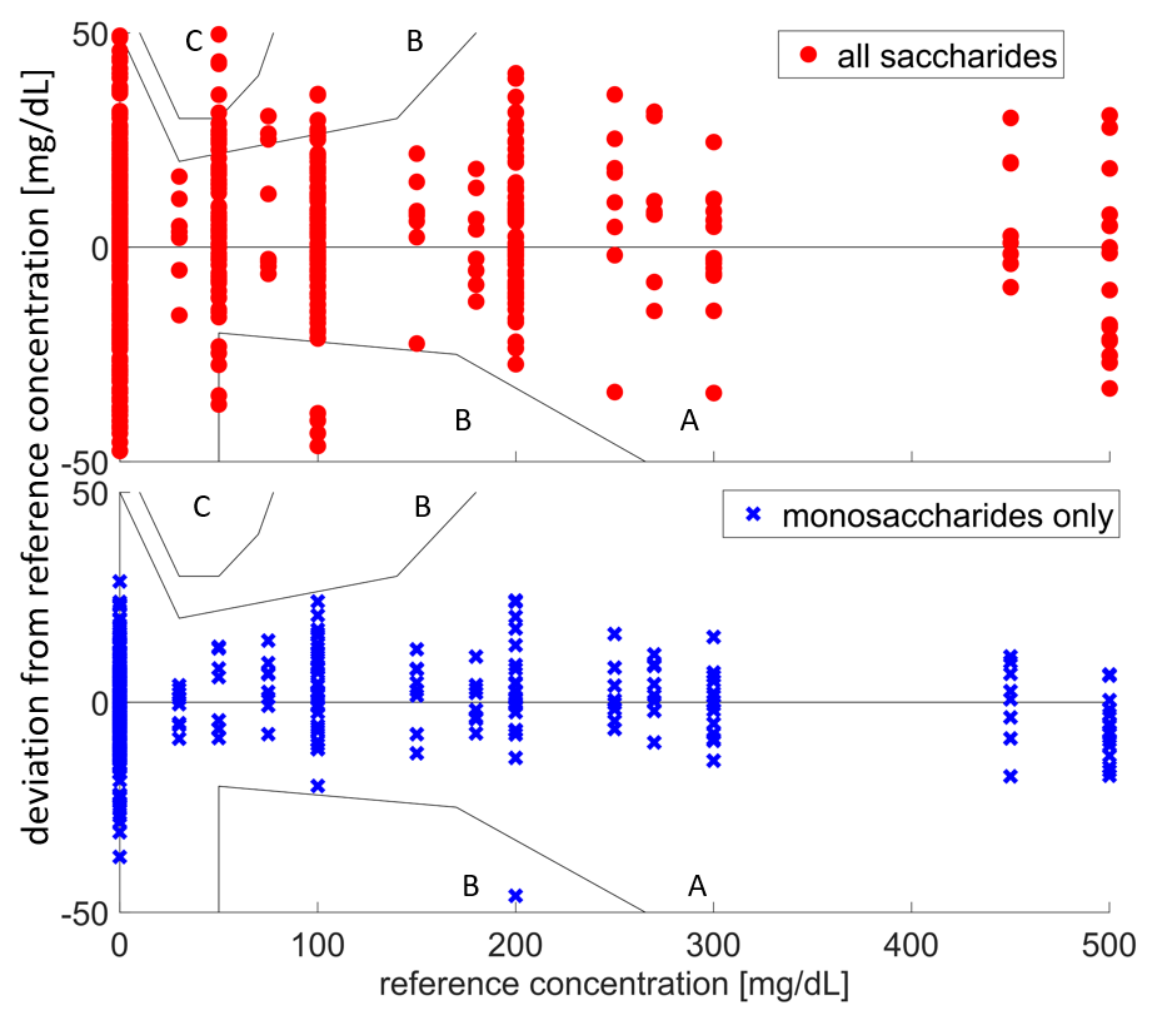

Figure 3.9.: Absolute deviation of predicted glucose concentration from reference concentration within a mixture of other monosaccharides and disaccharides (red) and monosaccharides only (blue) as predicted by PLS analysis (leave-one-cycle-out crossvalidation, 7 latent variables). 

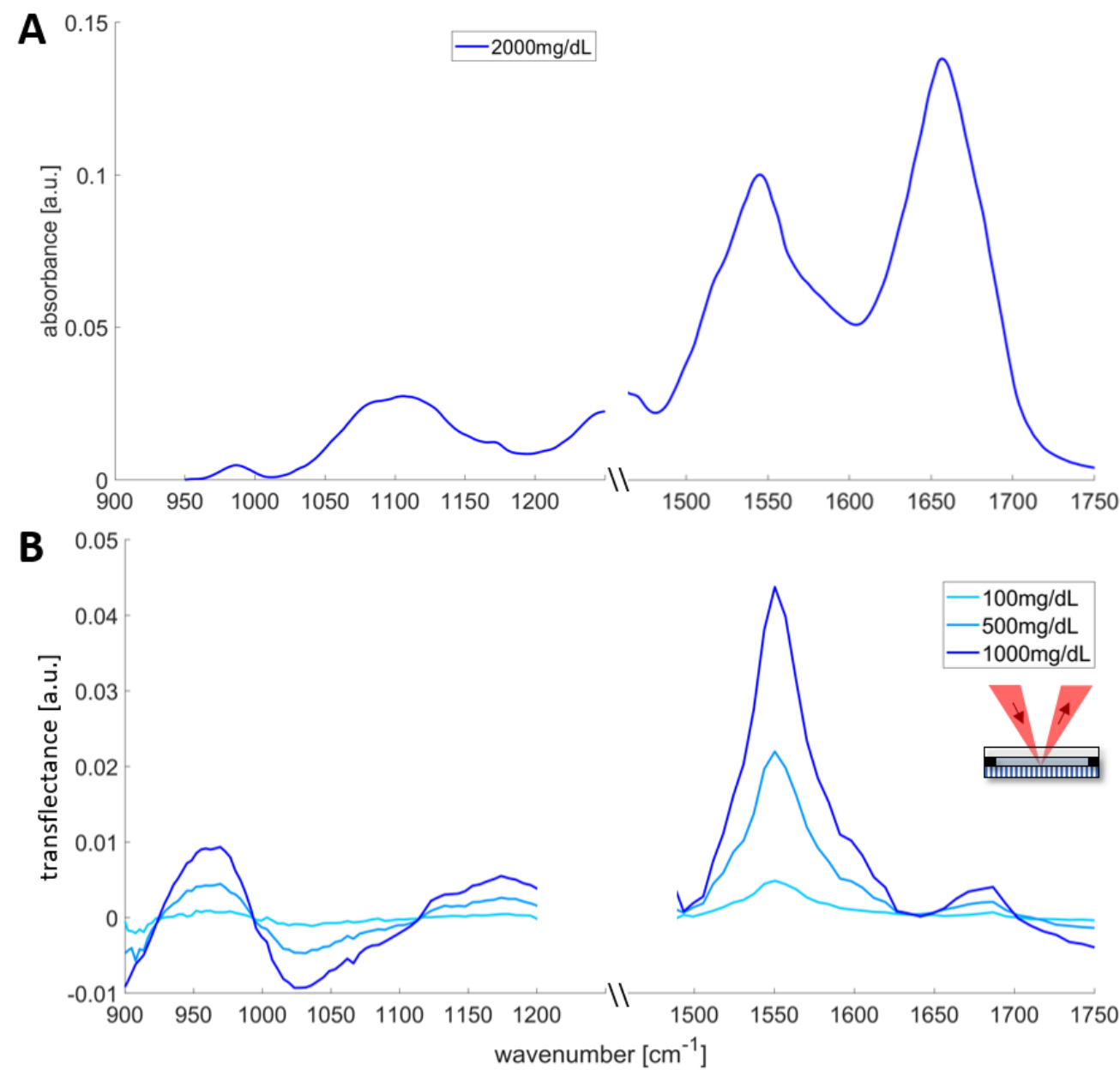

Figure 3.10.: (Decadic) absorbance and transflectance spectra of albumin. A: FTIR spectrum acquired in transmission of a dried albumin film [77], B: Spectra acquired in transflection geometry. Figure B adapted from Isensee et al. [25]. 


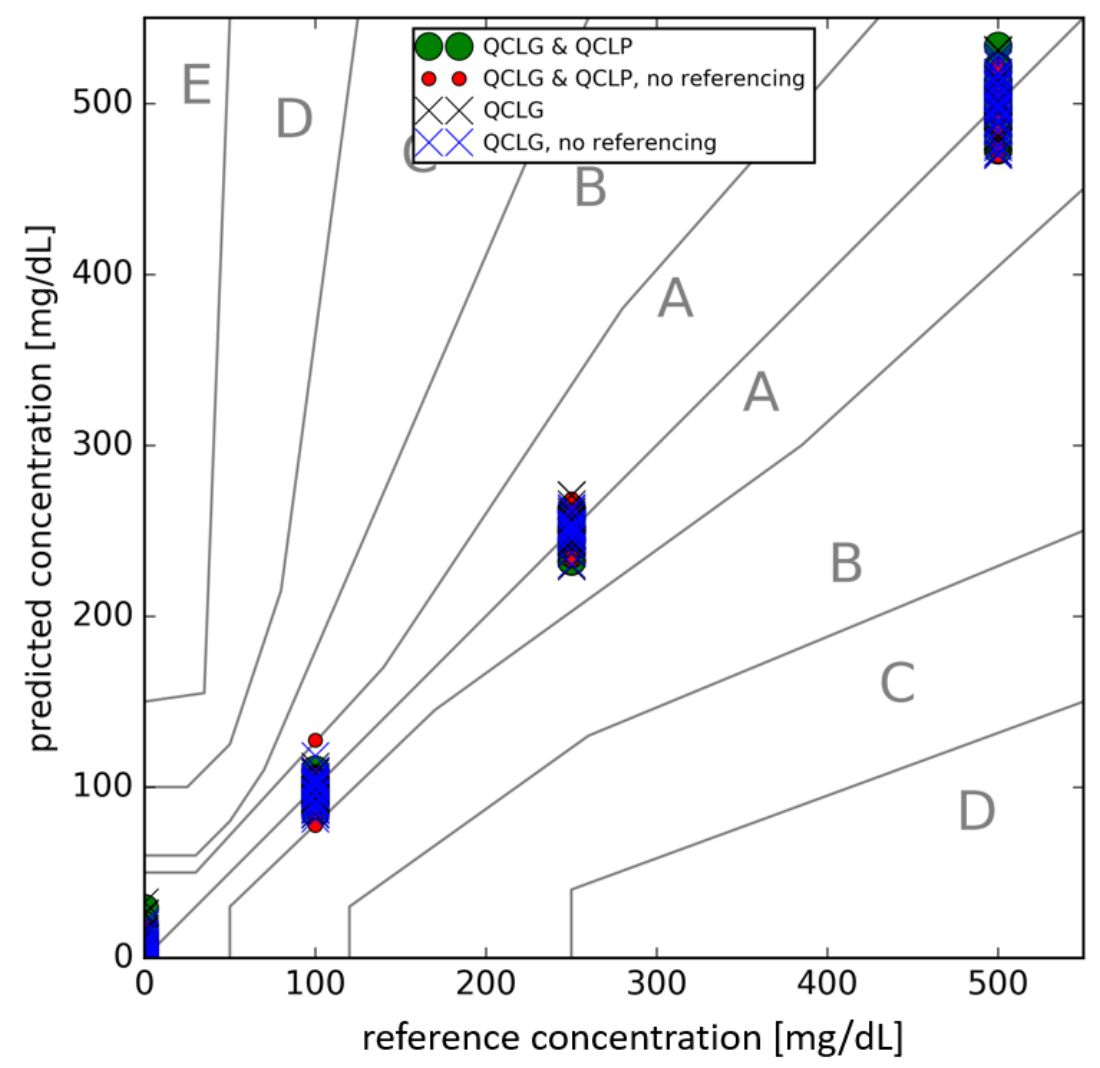

Figure 3.11.: Predicted glucose concentrations in mixtures of up to $1000 \mathrm{mg} / \mathrm{dL}$ albumin shown in a Parkes error grid, calculated with PLS analysis with and without water referencing, as well as with and without QCLP. All measurements are taken within one day (intra-day). 


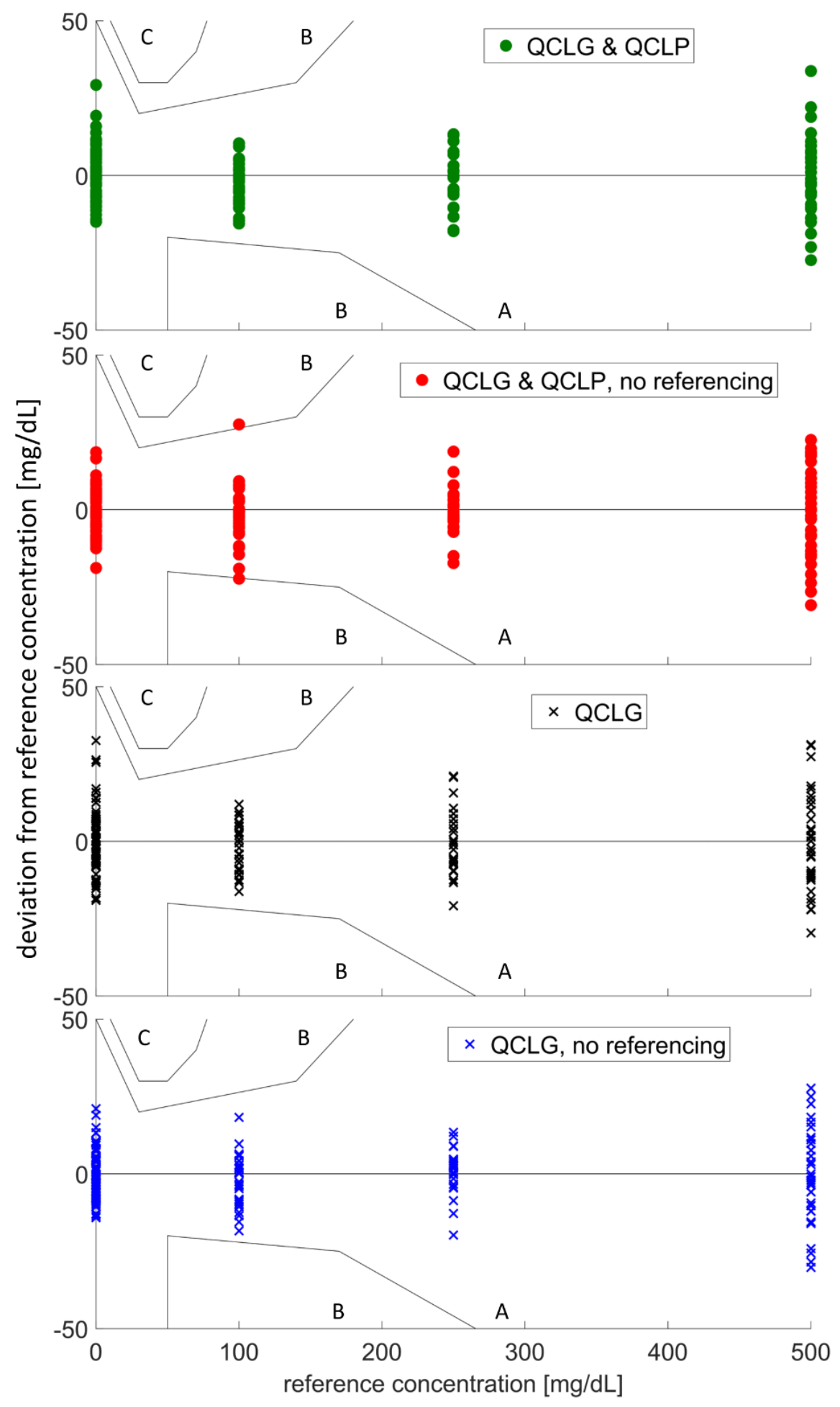

Figure 3.12.: Absolute deviation of predicted glucose values in the presence of up to $1000 \mathrm{mg} / \mathrm{dL}$ albumin calculated with PLS analysis with and without water referencing, as well as with and without QCLP. Nearly all predicted values are in the region A of the Parkes error grid. All measurements are taken within one day (intra-day). 


\section{Experiments and results}

\section{Impact of temperature}

The temperature of an uncooled in vivo sensor might drift by a few degrees depending on the temperature changes of the surrounding tissue. Therefore, the temperature dependence of the raw signal as well as the change in transflectance is investigated in vitro in the following. Experimentally, the signal obtained for glucose $(500 \mathrm{mg} / \mathrm{dL})$ and water is acquired by heating the sensor to a temperature around $45^{\circ} \mathrm{C}$ and then measuring transflection signals while the system's temperature falls back down to room temperatures. These heating cycles are repeated for several times in order to collect enough data (120 samples for water and glucose). Figure 3.13 shows examples for the raw signals obtained for water at different temperatures. The change of the raw signal with temperature is strongly dependent on the wavenumber and it is larger in the spectral region of QCLP, especially in regions where the water absorbance is quite small (around $1550 \mathrm{~cm}^{-1}$ and $1740 \mathrm{~cm}^{-1}$ ).

A theoretical simulation of the reflected signal (see Section 2.1.4) shows that there are different parameters that change with temperature as for example the layer thickness of the water layer, the refractive indices and the absorptivities of the optical components. Theoretical considerations (see Appendix A.1) show that the impact of the water layer thickness is the most prominent. As the temperature dependence of the water layer thickness additionally is the most uncertain one from the geometrical setup, a regression of the obtained signal with the water layer thickness left as free parameter is carried out (all input values are summarized in Table 2.3). A typical fit curve is shown in Figure 3.14. While the theoretical model (red) fits well to the acquired data (blue), there are still some differences present between experimentally measured and theoretically calculated signal. The deviations might arise from imprecise literature values for the refractive index, the absorptivity of water and variations in the reflectivity of the patterned gold layer, as well as the individual temperature dependence of these parameters. As shown in Section 2.1.4, the variation of numerical values from different references amounts to $1.6 \%$ and $4.3 \%$ for refractive index (real-part) and absorptivity, respectively. Given the lack of data for the temperature dependence of the refractive index of water $n_{2}$ (real part) a constant dependence of $\Delta n_{2} / \Delta T=-0.0011 / \mathrm{K}$ (interpolated average from data shown in Fig. 2.10 [52]) is assumed for all wavenumbers, which is probably not the case in nature.

Figure 3.15 shows the resulting water layer thicknesses $d_{2}$ with respect to temperature. As one would expect from thermal expansion of the surrounding sensor holder (as described in Section 2.1.4) a linear dependence is observable. A linear regression leads to a thermal expansion coefficient of $\Delta d_{2} / \Delta T=12.8(1) \mathrm{nm} / \mathrm{K}$. As discussed in detail in Sec. 2.1.4, this expansion is in a realistic range (between $0 \mathrm{~nm} / \mathrm{K}$ to $15.4 \mathrm{~nm} / \mathrm{K})$ to be caused by the expansion of the sensor housing under the assumption that the outer holder expands more than the inner parts and the pressure is partly upheld by the flexible silicone rings on top and below the layer stack. The deviation between the individual values for glucose (red) is probably caused by taking the data before the system is thermally equilibrated (see Appendix A.3). This was 


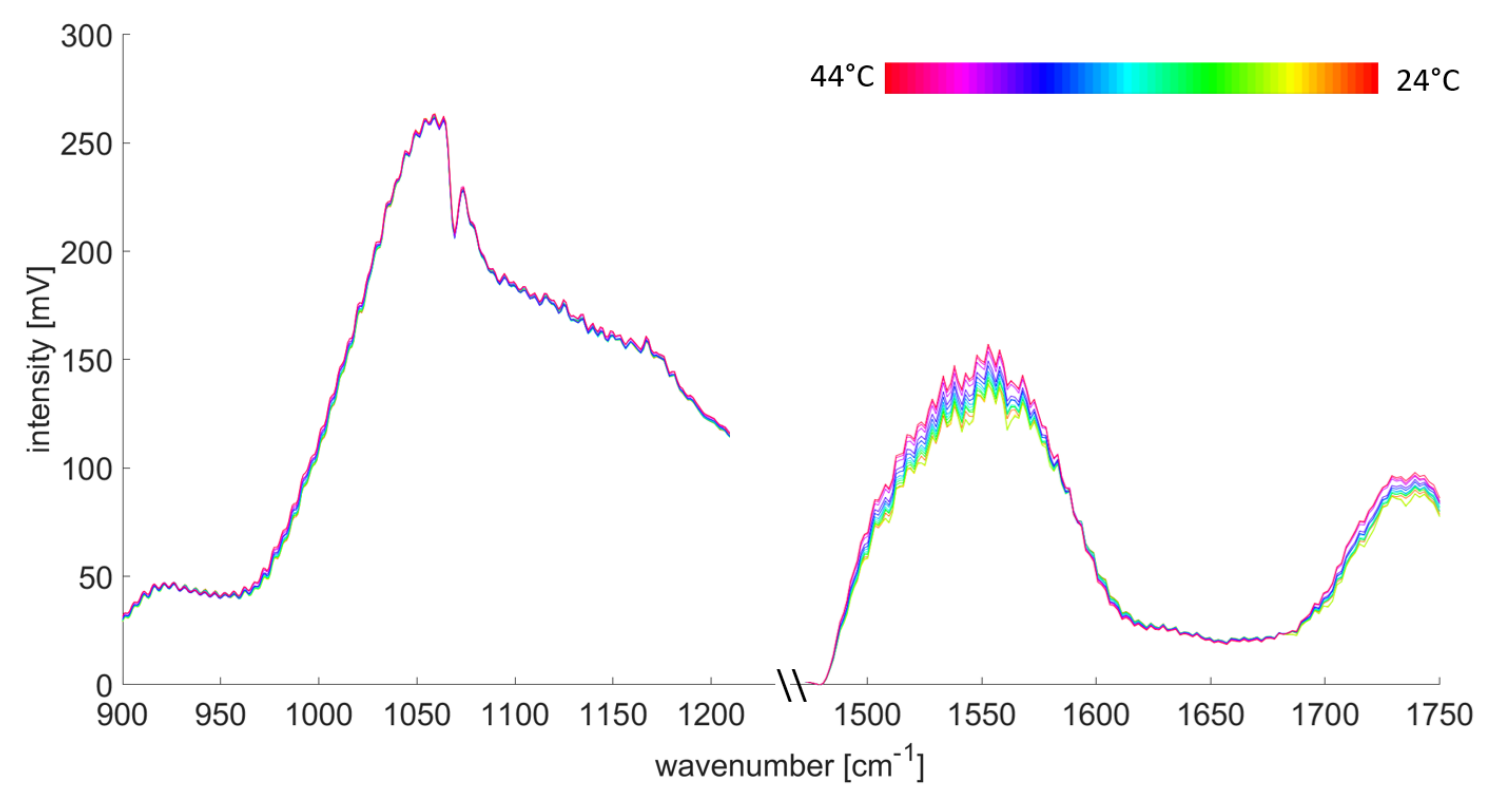

Figure 3.13.: Transflected signals $I_{\text {reflected }}$ at temperatures between $20^{\circ} \mathrm{C}$ to $45^{\circ} \mathrm{C}$ in the spectral range of QCLG $(\mathbf{A})$ and QCLP $(\mathbf{B})$. Figure adapted from Isensee et al. [25].

retrospectively recognized and could not further be investigated due to time reasons. It leads to a shift in read-out temperature compared to the real temperature in the measurement chamber as it is observed in Fig. 3.15. Except for this phenomenon, the slope of the lines is still the same as the one for water. This finding confirms the consistent temperature dependence of the water layer. It has to be pointed out that the impact of glucose absorptivity and refractive index on the signal and its temperature change is completely neglected in this consideration as the absolute concentration of glucose is four orders of magnitude smaller than the one of water (at a glucose concentration of $500 \mathrm{mg} / \mathrm{dL}$ ).

How does a temperature change affect the glucose prediction? To answer this question, transflection measurements of two glucose solutions $(0 \mathrm{mg} / \mathrm{dL}$ i.e. water and $500 \mathrm{mg} / \mathrm{dL})$ are performed at various temperatures between $25^{\circ} \mathrm{C}$ to $40^{\circ} \mathrm{C}$. As the two concentrations are acquired at different days and not at the exact same temperatures, there is no suitable water reference spectrum available for each individual measurement. Using the non-referenced signal for a glucose prediction with PLS leads to inaccurate predictions (as already observed in the day-to-day variance in pure glucose solutions). These inaccuracies are amplified by th change in temperature. To overcome this issue the predicted values have to be recalibrated and corrected with respect to temperature: First a PLS analysis on the unreferenced spectra at the two concentrations (using the dataset of glucose and albumin from Sec. 3.1.2 as training data) is done. Then at every temperature $\mathrm{T}$, the predicted concentration of the solution with $0 \mathrm{mg} / \mathrm{dL}$ glucose $\left(C_{\text {water,predicted }}(T)\right)$ is subtracted from the 


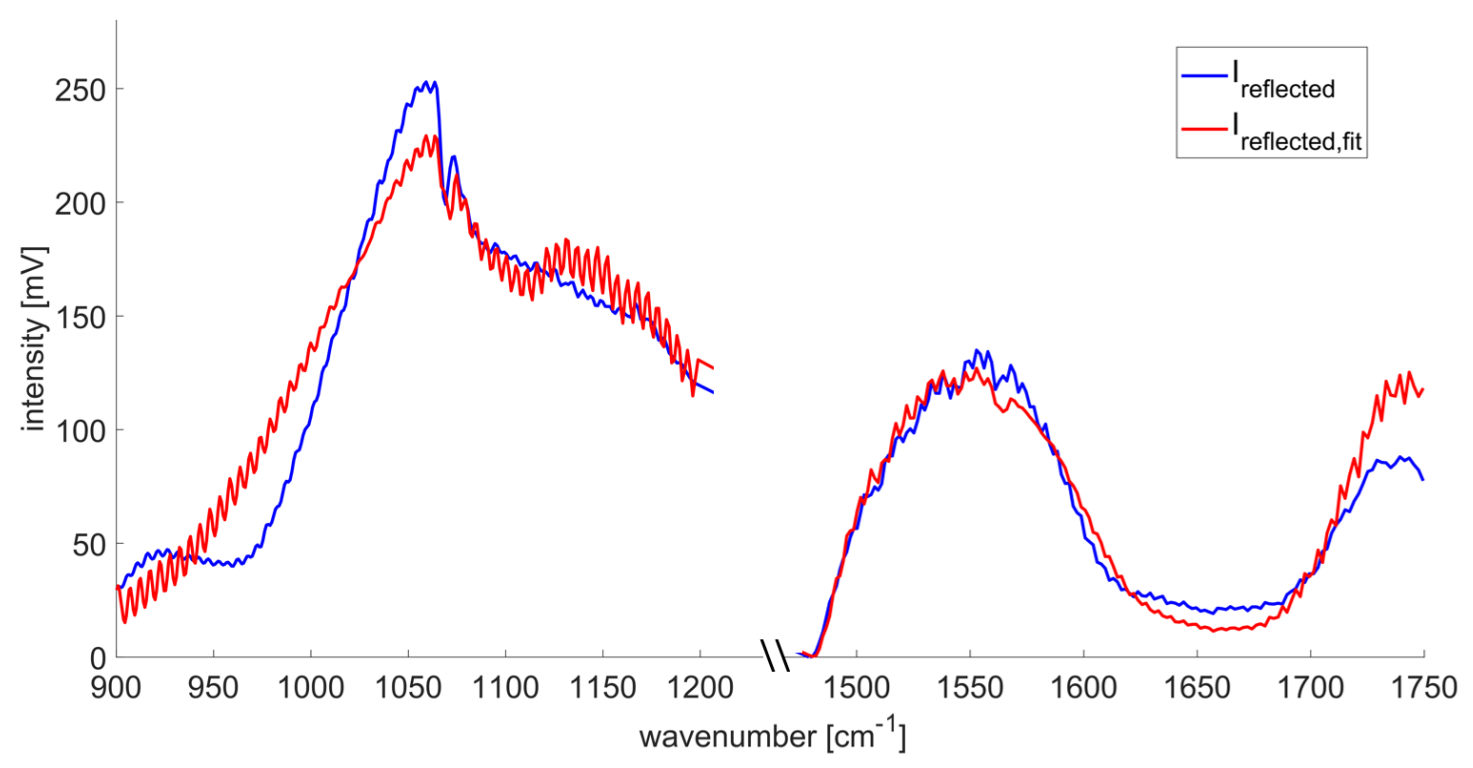

Figure 3.14.: Typical fit curve $I_{\text {reflected,fit }}$ (orange) of the theoretical calculated reflected intensity on the actually measured signal $I_{\text {reflected }}$ (blue) shown in the spectral region of QCLG $(\mathbf{A})$ and QCLP $(\mathbf{B})$.

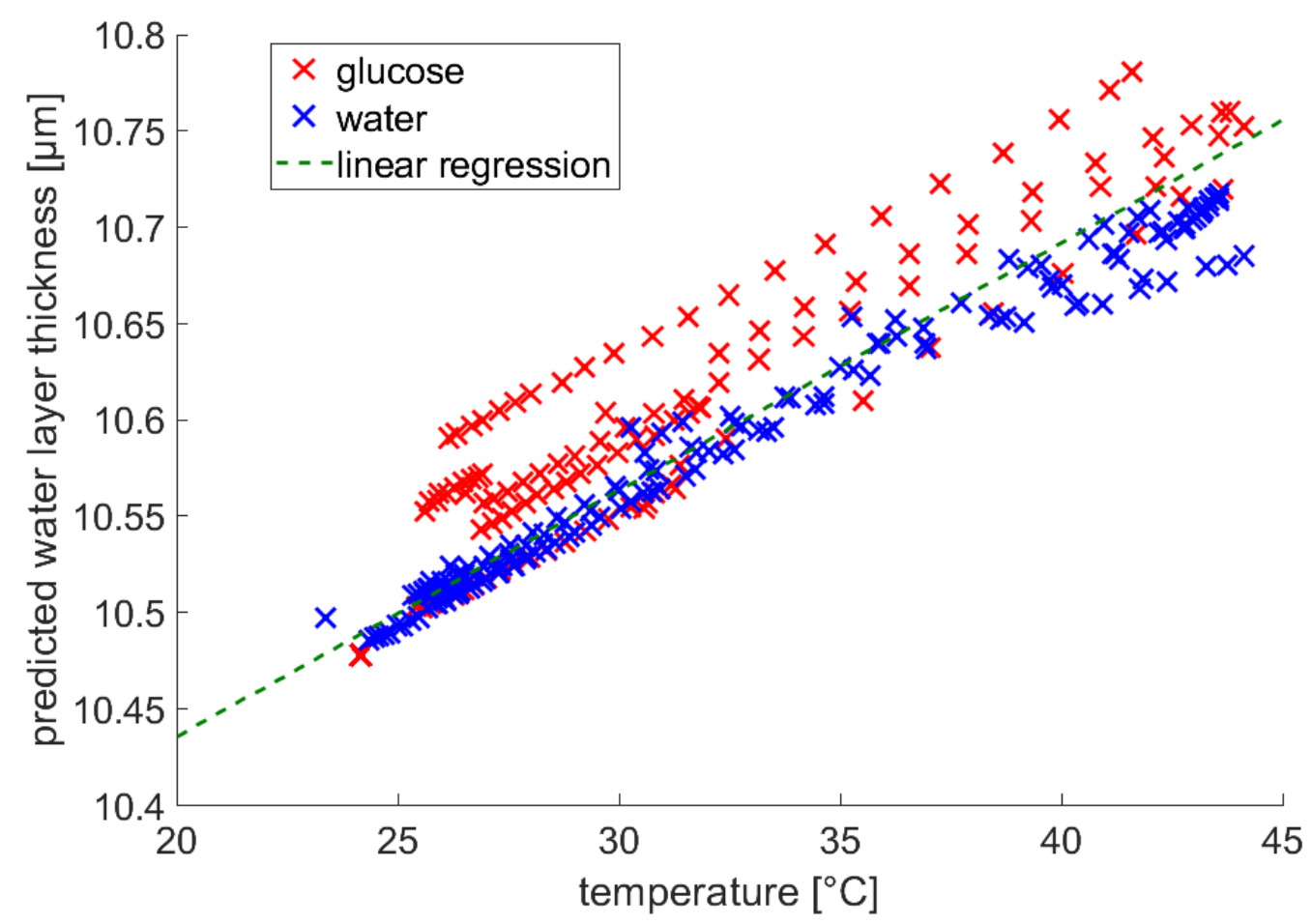

Figure 3.15.: Predicted water layer thickness $d_{2}$ with respect to temperature in water (blue) and glucose (red). The values show a linear increase with temperature of $\Delta d_{2} / \Delta T=12.8(1) \mathrm{nm} /{ }^{\circ} \mathrm{C}$. 
predicted concentration of the solution with $500 \mathrm{mg} / \mathrm{dL}$ glucose $\left(C_{\text {glucose,predicted }}(T)\right)$. For recalibration of the predicted concentrations, the deviation between the glucose prediction of one single measurement in the test set $\left(C_{\text {glucose,referencepoint,predicted }}\right)$ and the real glucose concentration $\left(C_{\text {glucose,referencepoint,real }}\right)$ is used as scaling factor for all predicted values. Thus, the overall correction is done according to

$$
C_{\text {glucose, corrected }}=\frac{C_{\text {glucose, predicted }}(T)-C_{\text {water, predicted }}(T)}{C_{\text {glucose,referencepoint,predicted }}-C_{\text {glucose,referencepoint,real }}} .
$$

The predicted corrected glucose values are shown in Fig. 3.16 with respect to temperature. The single measurement used for recalibration is marked in red. The values are calculated for both QCLs and QCLG only. A RMSEP for glucose of $18.5 \mathrm{mg} / \mathrm{dL}$ and $130 \mathrm{mg} / \mathrm{dL}$ is obtained using both lasers and QCLG only, respectively. This shows that by using the here introduced recalibration method, an acceptable sensitivity can be achieved even though no referencing on a water measurement was done. In contrast to the experiments done within mixtures of different substances, here, QCLP has a large effect on the glucose sensitivity. This leads to the conclusion that QCLP provides important information about the temperature and sensor conditions and thus is very helpful for the compensation of such sensor changes. 


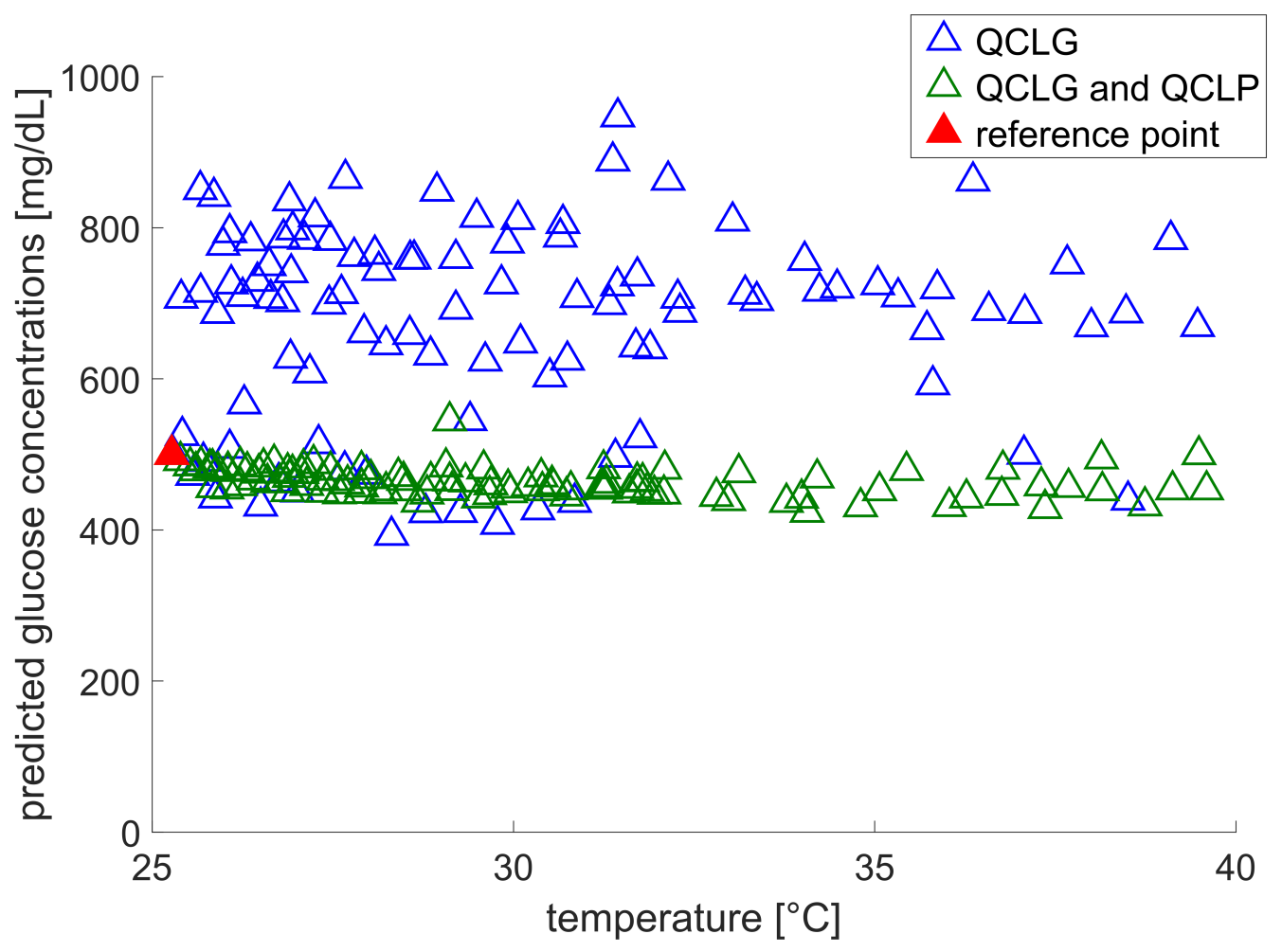

Figure 3.16.: Predicted glucose concentration with respect to temperature change and used QCLs for a reference concentration of $500 \mathrm{mg} / \mathrm{dL}$. Values are calculated by PLS analysis that is trained on a data set acquired at room temperature and the values are corrected with the predicted water concentrations at the respective temperatures. Additionally, all values are recalibrated by using the very first predicted value (indicated in red) to correct for an overall setup change in the timespan in between the two measurements (several months). The standard deviation of the predicted glucose concentration from $500 \mathrm{mg} / \mathrm{dL}$ amounts to $130 \mathrm{mg} / \mathrm{dL}$ and $18.5 \mathrm{mg} / \mathrm{dL}$ using only QCLG and both QCLs, respectively. 


\subsection{Glucose diffusion properties}

For the monitoring of a physiological glucose change in vivo the sensor's response time must be adequately fast (i.e. faster than $5 \mathrm{~min}$ ). The response time of the sensor is the sum of the time glucose needs to diffuse into the measurement chamber and the time needed for the spectral measurement itself. In this section, the timescales of the diffusion process will be addressed by numerical simulations and in vitro experiments. In addition, the impact of temperature on the diffusion time will be assessed.

The glucose diffusion time for the sensor is measured by abruptly changing the glucose concentration in the fluidic reservoir $C_{\text {reservoir }}$ and measuring the transflection signal from the measurement chamber over time (as described in Section 2.2.3). In order to get a high time resolution, the laser is set to a fixed wavenumber at the position of a glucose absorption peak of $1045 \mathrm{~cm}^{-1}$. Thereby the transflection signal corresponds to the glucose concentration inside the measurement chamber $C_{\text {chamber }}$ and a decrease (or increase if $C_{\text {reservoir }}<C_{\text {chamber }}$ ) in transflection signal can be observed during the diffusion process. An example of a typical measurement result is shown in Fig. 3.17 together with the corresponding result of the numerical simulation (see also Section 2.2.2).

In general, diffusion through a membrane is a complex process that is dependent on various properties of the system. As discussed in Section 2.2.1, the diffusion dynamics depend on parameters such as the mass and size of the molecules, the viscosity of the substance, the porosity and pore sizes of the membrane, the temperature as well as the diffusion distance.

When looking at the sensor with the silicon wafer only (without an additional filtration membrane attached), the diffusion process should be dominated by diffusion in free solution as the holes in the silicon are very large $(10 \mu \mathrm{m})$ compared to the size of the molecules. Hence no size-specific filtering effect from the silicon is expected. The diffusion time should behave according to the Stokes-Einstein equation (see Equation 2.34) and thus depend on the molecular mass $M$ according to $\tau \propto M^{1 / 3}$.

The results of a measuremnt with glucose, maltodextrin and albumin (see Table 3.4 ) leads to an average glucose diffusion time (from 12 measurements) of $18(8) \mathrm{s}$ whereas the simulations suggest a diffusion time of $32 \mathrm{~s}$. The measured diffusion times for maltodextrin and albumin are in the same value range. Using the measured glucose diffusion time the theoretically expected diffusion times of maltodextrin and albumin can be calculated from the Stokes-Einstein equation (see Table 3.4, column 4): While the measured diffusion time of maltodextrin meets the expected time within its error, the diffusion time measured for albumin is about $100 \mathrm{~s}$ faster than expected.

According to the Stokes Einstein equation there is also an inverse dependence on the temperature. This effect is demonstrated in a second experiment where the glucose diffusion time is acquired (see Figure 3.18, empty symbols). However, no inverse dependence on temperature $T$ is observable by a $1 / T$ fit.

An additional ultrafiltration membrane is needed for in vivo since many other sub- 


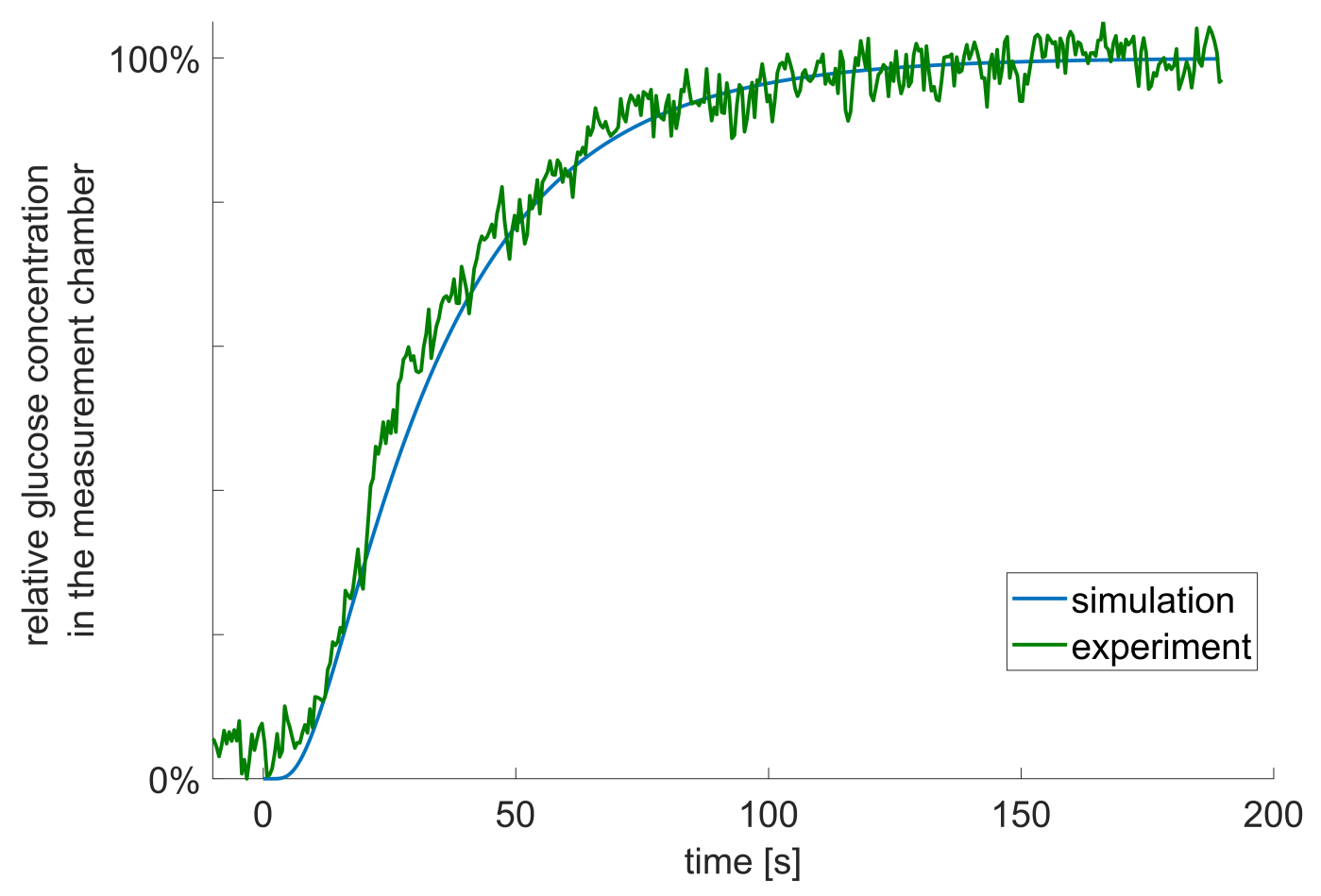

Figure 3.17.: Glucose concentrations in the measurement chamber after changing the concentration in the fluidic reservoir (at time $0 \mathrm{~s}$ ). The simulation (green) was done with geometrical parameters as used in the experiment (silicon layer thickness: $200 \mu \mathrm{m}$, water layer thickness $10 \mu \mathrm{m}$ ). The experimentally measured curve was acquired by a fixed frequency measurement with QCLG at a wavenumber of $1045 \mathrm{~cm}^{-1}$ and normalized to the equilibrium concentration.

Table 3.4.: Measured average diffusion times of 12 measurements per substance (room temperature, no additional filtration membrane). ${ }^{1)}$ : Expected value extrapolated from the glucose diffusion time and the assumed $\tau \propto M^{1 / 3}$ dependence.

\begin{tabular}{cccc}
\hline substance & average molecular weight & diffusion time $[\mathrm{s}]$ & expected diffusion time $[\mathrm{s}]^{1}$ \\
\hline Glucose & $0.18 \mathrm{kDa}$ & $18 \pm 8$ & $(18)$ \\
Maltodextrin & $1 \mathrm{kDa}$ & $24 \pm 11$ & 32 \\
Albumin & $66 \mathrm{kDa}$ & $22 \pm 8$ & 129 \\
\hline
\end{tabular}




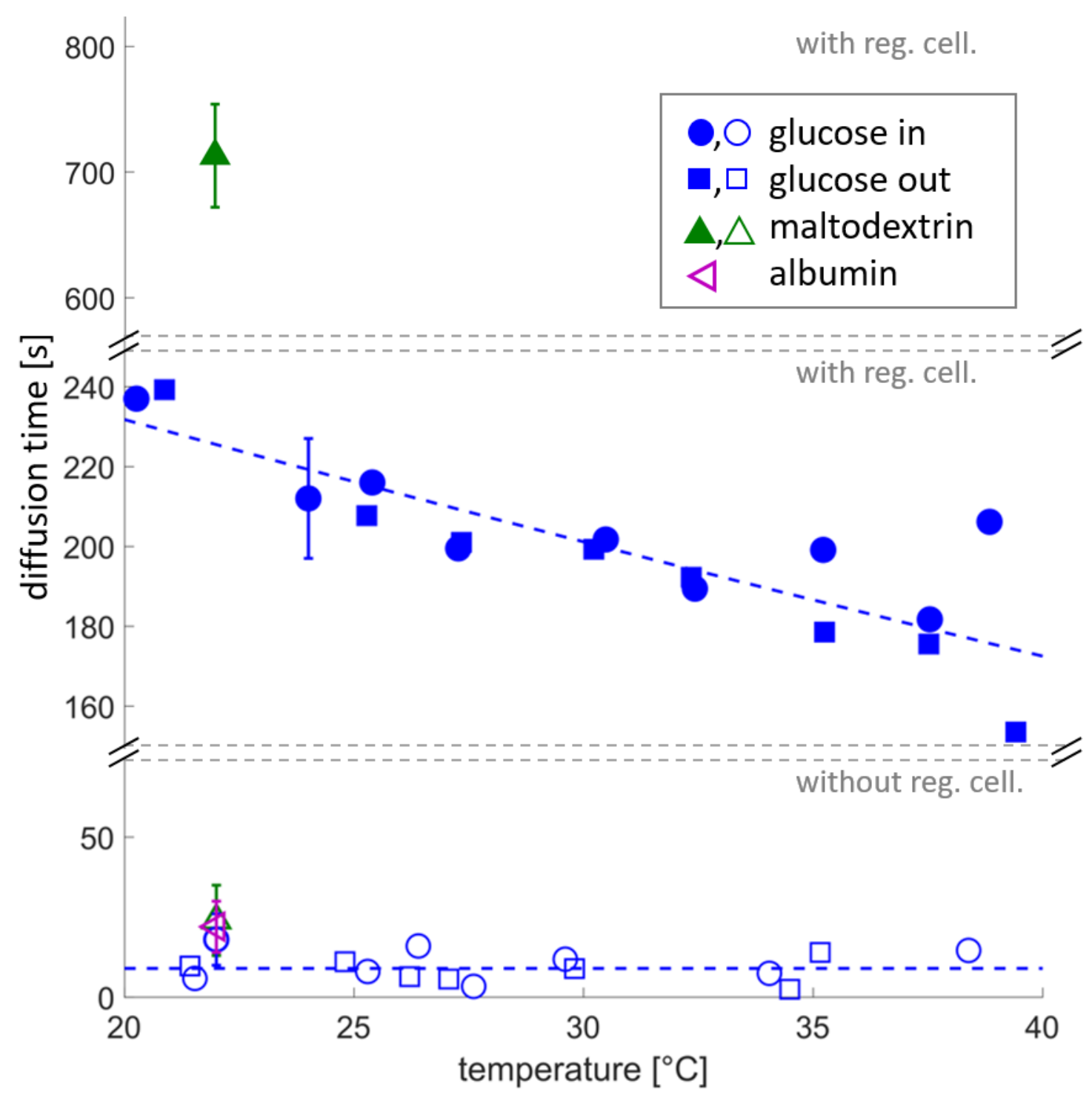

Figure 3.18.: Overview of temperature dependence of diffusion times measured for different substances with (filled symbols) and without (empty symbols) ultrafiltration membrane. Circles indicate measurements where glucose diffusion direction is into the measurement chamber and squares indicate measurements where the diffusion direction is out of the measurement chamber. The dashed lines show the fitted $1 / T$ dependence of the diffusion time. Figure adapted from [25]. 


\section{Experiments and results}

stances (especially proteins) besides glucose are present in the surrounding medium. Its purpose is to prevent proteins and other macromolecules from entering the measurement chamber, and thus from attaching to the surface and impacting a longterm application of the sensor. It is expected that the glucose diffusion time will be elongated significantly as the membrane serves as filtering barrier. In addition to diffusion in free solution, also membrane specific processes will play a role. These comprise for example the adhesion of molecules inside the membrane's pores, the membrane's diffusion resistance caused by its porosity (e.g. death ends inside the structure) and charging effects on the surfaces. This complex interplay of processes was theoretically described by Renkin [78] and experimentally verified by Beck and Schultz [79]. They used a semi-empirical approach that leads to an effective diffusion coefficient $D_{\text {eff }}$ according to

$$
D_{\text {eff }}=D\left(1-\frac{R_{s}}{R_{p}}\right)^{2}\left[1-2.104\left(\frac{R_{s}}{R_{p}}\right)+2.09\left(\frac{R_{s}}{R_{p}}\right)^{3}-0.95\left(\frac{R_{s}}{R_{p}}\right)^{5}\right]
$$

with $R_{s}$ being the radius of the particle, $R_{p}$ being an effective pore size of the membrane and $D$ being the diffusion coefficient of the particles in free solution. This formula shows that in first order the diffusion time follows the dependence $\tau \propto \frac{1}{D\left[1-R_{s} / R_{p}\right]^{2}}$. Hence, the temperature dependence of the diffusion time is in first order given by the temperature dependence of the diffusion coefficient $D$ which follows the Stokes-Einstein equation: $\tau \propto 1 / T$ as shown earlier.

The effect of an such a membrane is investigated experimentally for the diffusion of glucose and maltodextrin using a regenerated cellulose membrane with a nominal weight cutoff of $25 \mathrm{kDa}$ (Nr. 15004, Reichelt Chemietechnik). The result is shown in Figure 3.18. For glucose a diffusion time of 214(15) $\mathrm{s}$ is obtained. As the diffusion time of albumin is too long to measure experimentally (a huge amount of albumin solution and a very long measurement time would be needed) the filtering effect is proven by a measurement of maltodextrin. Its larger molecular mass compared to glucose leads to a significantly longer diffusion time of 713(41) s.

Further, the effect of temperature is measured between $20^{\circ} \mathrm{C}$ to $40{ }^{\circ} \mathrm{C}$. While no temperature dependence was observed in the measurements with silicon wafer only, the measurements with additional membrane show the expected $1 / T$-dependence (see dashed line in Fig. 3.18).

To conclude this section: a spectroscopic measurement time smaller than $100 \mathrm{~s}$ in addition to the measured diffusion time of $214 \mathrm{~s}$ enables reasonable fast sensor response time of $5 \mathrm{~min}$. This measurement time scale corresponds to e.g. the time of 4 laser sweep scans which, as shown in Chapter 2.1, easily satisfy the sensitivity requirements for an in vivo sensor.

\subsection{Long-term stability}

In order to evaluate the sensors performance over a long period of time, an experiment over 42 days is performed. Within this time, periodic spectral measurements as 
well as glucose diffusion experiments (at fixed frequency) are carried out. To imitate in vivo conditions the solutions contained $1000 \mathrm{mg} / \mathrm{dL}$ of albumin. The corrected glucose predictions of solutions containing $500 \mathrm{mg} / \mathrm{dL}$ glucose and $1000 \mathrm{mg} / \mathrm{dL}$ albumin are shown in Figure 3.19 A. The PLS analysis is trained with a training data set taken 5 days before the start of the longterm measurement containing non-referenced spectra of different mixtures of glucose (concentrations: 0-500 mg/dL) and albumin (concentrations: $0-1000 \mathrm{mg} / \mathrm{dL}$ ). To correct for the day-to-day variance in the data, the predicted values are recalibrated. This is done by subtracting the predicted value for a reference solution (in this case containing $1000 \mathrm{mg} / \mathrm{dL}$ albumin) taken at the same day. Further the glucose prediction of the very first measurement (marked in red) was used for recalibration of the glucose predictions according to Equation 3.1. The diffusion times are acquired the same way as in Section 3.2 and are shown in Fig. $3.19 \mathrm{~B}$. The different symbols indicate measurements where the glucose diffusion direction points into the measurement chamber (circles) and out of the measurement chamber (squares). The average diffusion time with additional regenerated cellulose membrane is $221(9) \mathrm{s}$. There is no significant overall drift or slowdown observable (change rate of median is equal to zero within $2 \sigma$ ) and thus a harmful longterm effect from albumin can be excluded. Further the RMSEP of glucose is with $12.4 \mathrm{mg} / \mathrm{dL}$ well below $3 \%$ and does not show a significant worsening with measurement time. 


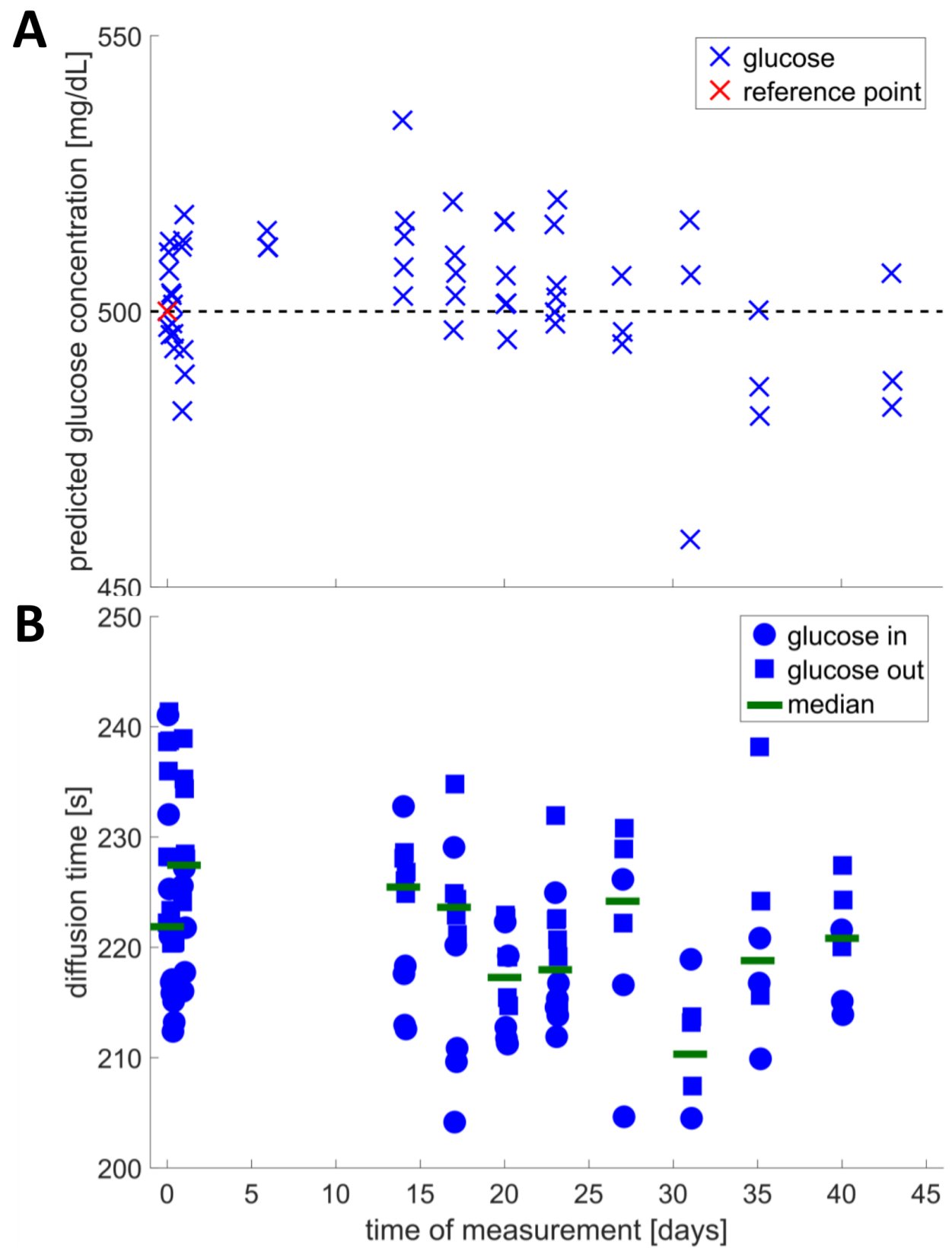

Figure 3.19.: A: Glucose prediction during long-term measurement obtained by PLS regression with 6 latent variables and a training data set taken 5 days before the start of the longterm measurement. The predicted values for glucose solution with a concentration of $500 \mathrm{mg} / \mathrm{dL}$ are corrected with a prediction from a reference substance and scaled by the very first prediction value (marked in red). B: Glucose diffusion times calculated from longterm experiment in the presence of $1000 \mathrm{mg} / \mathrm{dL}$ albumin in solution. Circles indicate measurements where glucose diffusion direction is into the measurement chamber and squares indicate measurements where the diffusion direction is out of the measurement chamber. Figure adapted from Isensee et al. [25]. 
4. Discussion 


\section{Discussion}

The development of QCLs enabled new prospects for MIR spectroscopic investigation of liquid samples in the last two decades. Using the high spectral power density of QCLs the measurements got much faster and the layer thicknesses became larger and thus easier to handle. Various groups investigated the sensitivity of this reagent-free method for the quantitative glucose measurement in vitro [11$13,15,16,18,22,80-86]$ as well as in vivo [14].

In this thesis, the theoretical and experimental basics of quantum cascade laserbased glucose measurements in the context of a future continuous glucose monitoring implant are investigated. Due to its compactness, size, biocompatibility, robustness and potential to be implantable, a transflection measurement approach is found to be most promising for this endeavour. The optical mechanisms in transflection are investigated theoretically and experimentally and it is shown that the achieved glucose sensitivity based on transflectance in pure glucose solutions as well as in complex solutions with other substances suffices the medical directives for continuous glucose monitoring. The influence of a temeperature change on the optical signal is investigated in detail and it is shown, that the temperature-related change in water layer thickness in the measurement chamber has the highest impact on the optical signal.

The glucose diffusion properties into and out of the sensor are evaluated using finite-volume simulations and experimental diffusion measurements. A response time below 5 min can be achieved, which meets the requirements for the desired sampling rate to monitor physiological glucose concentration changes in vivo.

The long-term stability of glucose sensitivity and glucose exchange is successfully demonstrated in vitro by a long-term experiment over a time period of 42 days. This finding highlights the prospect of using the transflection opto-fluidic interface in a future long-term implant.

Table 4.1 compares the demonstrated results to other work using QCLs with respect to different measurement parameters such as the type of QCL, the wavenumber range, the environment of the substance and the concentrations measured [20]. In Figure 4.1 a visualization of the achieved glucose sensitivities related to the respective mean glucose concentration is presented. All values except for the ones of this work (blue circles) are measured in transmission geometry. This is probably due to the more straight forward measurement design in transmission measurements. In terms of sensitivity the results measured in transflection are comparable or even better than the ones measured in transmission. The empty symbols indicate measurements done with fixed frequency QCLs (e.g. DFB-QCLs) whereas filled symbols indicate measurements where tunable QCLs (e.g. EC-QCLs) were used. All demonstrated measurements show accuracies better than $10 \%$ of the average glucose concentration in solution, whereby it is of note that all measurements with sensitivities better than $1 \%$ were made with a tunable QCL. It appears therefore legitimate to conclude that the use of tunable QCLs is preferable over single fixed-frequency QCLs for the investigation of body fluids such as glucose. However, EC-QCLs based on their tuning mechanism are "complex to build, requiring careful alignment and high-quality antireflection coatings." [87]. DFB-QCLs in contrast are "very compact 
(a few millimeters in length) and can easily be microfabricated in large quantities" [87]. Further, also arrays of different DFB-QCLs with the individual QCLs emitting at different wavelengths are reported (eg. Lee et al. [87]). Using such arrays or even just a few single DFB-QCLs would combine the spectral advantage of EC-QCLs with the small size simplicity of DFB-QCLs.

\section{Spectral measurement and measurement timescales}

As shown in Section 3.1, a high glucose sensitivity and selectivity can be achieved using the data from the spectral range of a tunable EC-QCL from $900 \mathrm{~cm}^{-1}$ to $1200 \mathrm{~cm}^{-1}$. When it comes to in vivo application, however, a compact, robust and easily adjustable sensor is required. Hence, the use of several small fixed frequency QCLs (such as DFB-QCLs or Fabry-Perot QCLs) emitting at the glucose-specific wavenumbers is a preferred option. If individual wavenumbers are to be used, then the question arises which wavenumbers to choose. To find the most relevant wavenumbers for the quantification of glucose, the EC-QCL spectra are artificially split up and just a few distinct wavenumbers are used for the prediction of glucose concentration. For the quantification of the importance of each wavenumber an evolutionary algorithm is used (see Section 2.1.6). Table 4.2 provides an overview on the most relevant wavenumbers and the corresponding RMSECVs for an optimization on the data set of a mixture of glucose and other monosaccharides. It is justified to neglect the impact of other disaccharides because they typically don't occur in the interstitial fluid [72-74]. With this assumption, a RMSECV around $15 \mathrm{mg} / \mathrm{dL}$ with only 5 distinct wavenumbers (assuming a bandwidth of $4 \mathrm{~cm}^{-1}$ ) can be achieved. This also reduces the measurement time significantly from $68 \mathrm{~s}$ ( 4 scans without dead time) to $5 \mathrm{~s}$ (corresponding integration time of each wavelength: $1 \mathrm{~s}$ ). In Figure 4.2 one can see that nearly all selected wavenumbers lay in a glucose absorbing region. Surprisingly, the pronounced glucose peak around $1080 \mathrm{~cm}^{-1}$ does not seem to have much importance for the glucose specificity under these conditions.

One of the next steps towards an in vivo sensor would be to experimentally investigate the possibilities of such an DFB-QCL array. It would further be conceivable that all individual DFB-QCLs would lase at the same time with different modulation frequencies. This way, with the help of various micro fabricated lock-in amplifiers at the respective modulation frequencies, the individual wavenumber-information could be filtered out without the need for an optical filter or spectrometer, and the overall measurement time could be decreased even more. However, it has to be pointed out that measurement time is not the only time-limiting factor: the glucose exchange time of the sensor (in the range of minutes) is even more relevant as it lays in the range of few minutes. The overall measurement time should not be longer than the timescale of physiological glucose change which is in the range of some minutes. As shown in Section 3.2 when using a ultrafiltration membrane, which is essential for a future in vivo use, in combination with the silicon wafer we measure glucose diffusion times in the range of 4 minutes. With a measurement time of some seconds the overall time delay would be smaller than 5 minutes and, hence, 


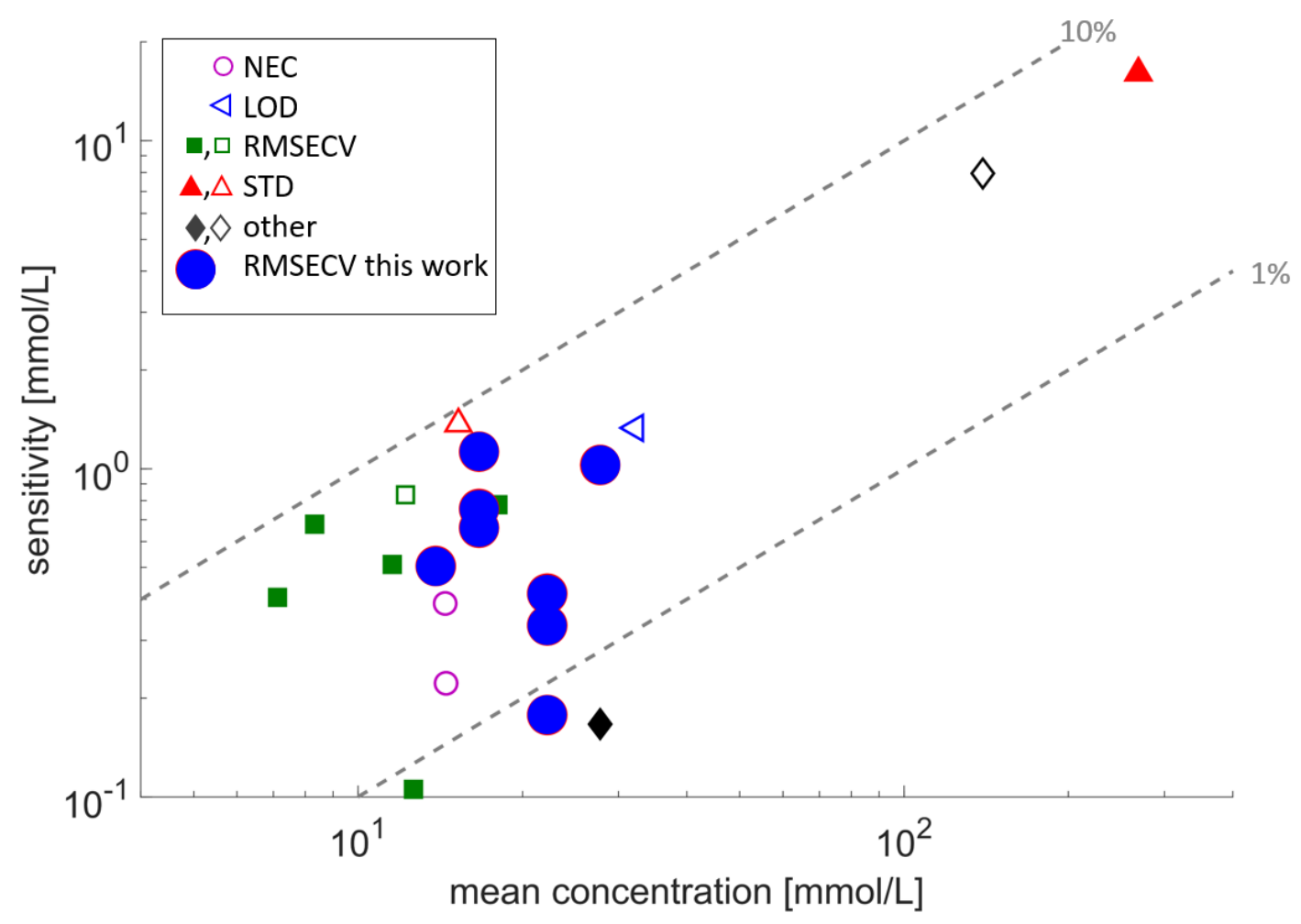

Figure 4.1.: Glucose sensitivities achieved in aqueous environments based on transmission (data taken from references in Table 4.1) and transflection (this work) measurements. The different symbols indicate different evaluation metrics for quantification of sensitivity: NEC: Noise equivalent concentration; LOD: Limit of detection; RMSECV: Root mean squared error of cross validation; STD: Standard deviation; other: other metrics such as root means squared error of prediction or absolute deviation from reference value. Filled symbols indicate measurements done with a tunable QCL whereas empty symbols indicate measurements done with a fixed-frequency QCL. Also 1\% and 10\% accuracy lines (dashed) are shown. Figure adapted from Isensee et al. [20]. 


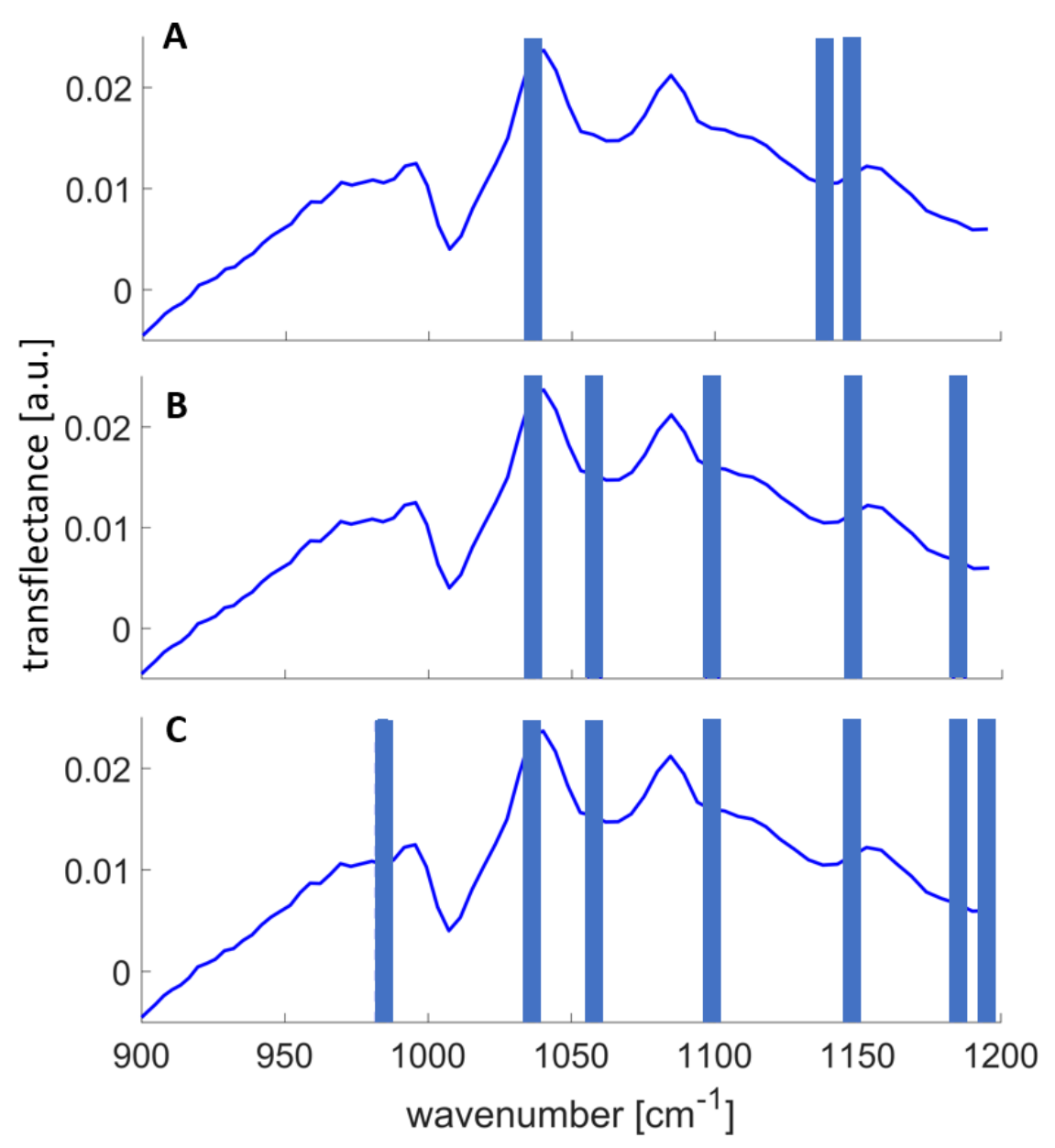

Figure 4.2.: Relevant wavenumbers for the determination of glucose concentration that are selected by an evolutionary algorithm. This is done with A) 3 distinct wavenumbers, B) 5 distinct wavenumbers and C) 7 distinct wavenumbers. The glucose transflectance spectrum of a solution of $500 \mathrm{mg} / \mathrm{dL}$ glucose is shown in blue for comparison.

sufficiently short for physiological glucose changes.

\section{Impact of temperature}

The response to a temperature change is another important aspect to look at as the temperature in the body can easily change by a few degrees depending on the location and the surrounding of the sensor. The experimental investigation of the signal change with temperature presented in Sec. 3.1.2 shows that quite a large effect is observable in the spectra. In contrast to what is observed in a transmission geometry (see e.g. [17]) the signal does not change linearly with temperature. Theoretical calculations of the reflected optical signal shown in Sec. 2.1.4 indicate that the main impact is the change in water layer thickness due to thermal expansion of 


\section{Discussion}

the sensor's stainless steel mounting. This effect is also seen in the investigation of the single contributions (see Sec. A.1) where, depending on the spectral position the effect of a change in layer thickness leads to a change in transflectance that is up to one order of magnitude larger than the effect of the other temperature-dependent quantities. However, this whole consideration has to be taken very carefully, as e.g. the references for the temperature dependence of the refractive index of water are relatively vague, and no real experimental data is found in the spectral region between $800 \mathrm{~cm}^{-1}$ to $1600 \mathrm{~cm}^{-1}$. Nevertheless it could be shown in Sec. 3.1 .2 that the effect of temperature on the transflectance can be corrected by a referencing procedure where water is measured at the same temperature and used as a reference. Using this procedure a relative RMSEP for glucose of $3.7 \%$ is achieved at concentrations of $500 \mathrm{mg} / \mathrm{dL}$. It is conceivable that this value still contains some errors as e.g. by the mounting position of the temperature sensor: In the setup used in this thesis it was not mounted directly at the position where the optical interaction takes place, i.e. inside measurement chamber itself, it instead was mounted on the stainless steel holder some millimeters apart from the measurement chamber. This presumably leads to an error in the temperature reading. To conclude, the correction procedure is a reasonable solution for a correction of the temperature drift in vitro. However, for the applicability in vivo, an additional reference measurement would lead to a doubling of the measurement time on the one hand and on the other hand would not be practically feasible with water as reference fluid in the same measurement chamber.

To overcome this impracticality we propose a sensor with an integrated reference chamber: The original measurement chamber is split up by a micro-fabricated well into two chambers, one being in exchange with the reservoir outside the sensor via the holes in the silicon wafer, and the other one being filled with a reference fluid (as e.g. water) and sealed from the surrounding (no holes in the silicon wafer). The beam is split up beforehand and detected by two separate detectors. This way either a direct analogous referencing of the raw signals or a referencing done the way described in Section 3.1.2 is possible. By this approach also the vague temperature measurement is redundant as the reference is taken under the exact same conditions (and temperature) as the measurement of the sample.

In the following an outlook and discussion on the possible use of the opto-fluidic interface in an in vivo implant will be given.

\section{Integration into an implant and miniaturization}

The overall prospect is the integration of the investigated IR-optical-fluidic sensor interface into a fully implantable device that contains all components needed for a measurement. It contains a QCL, a detector, beam shaping optics, the IR-opticalfluidic interface, an energy source, such as a rechargeable battery as well as a control unit with a transmitter that controls the measurement process, the energy management as well as the wireless transmission of the signal to an external receiver. The device could then be implanted somewhere in the interstitial fluid as e.g. in the 


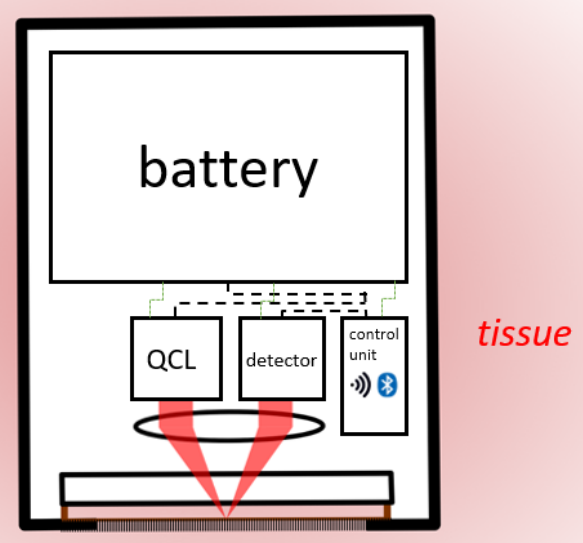

Figure 4.3.: Integration into a future implant consisting of different compartments: one or more QCLs as radiation source, beam shaping optics, an optofluidic interface, a battery as energy source, a control unit for signal filtering, data acquisition, energy management and communication with an outer receiver.

adipose tissue in the belly, and measure continuously over a long time without a need for the patient to interact with it. It could then be wirelessly coupled via bluetooth to an external receiver or insulin pump, that processes the data and autonomously reacts to the glucose information by e.g. inserting the right amount of insulin. Figure 4.3 exemplarily shows a sketch of such an implant.

In order to be able to implant the sensor in vivo following conditions must be met:

- The setup must be biocompatible and integrable in vivo

- The energy requirement of the sensor must be met

- The acquired data has to be handled and communicated to an external receiver

- The setup's size must be sufficiently small, i.e. in the range of several centimeters

- The setup must be long-term stable and must have a long lifetime (several months) 


\section{Discussion}

In the following these points will be discussed in detail.

\section{In vivo environment}

The sensor surface has to be made out of a biocompatible material or has to have a biocompatible coating. Therefore, the proven materials and techniques used in other fields as e.g. in pacemakers, can be adapted for the implant's housing. The more critical part is the infrared-optic-fluidic interface. The regenerated cellulose membrane is reported to be biocompatible [88, 89], also silicon and diamond as well as gold are biocompatible materials. Only the antireflective coating which contains ZnSe (rated as toxic by Regulation (EC) No.1907/2006) is problematic. So far, no solubility in water was experienced by us, however, there should be further investigations on the biocompatibility of this material. Another option would be to design a new coating made of biocompatible materials such as e.g. polyethylene [90]. Further, the immune response of biological organisms to the specific implant as well as the fibril encapsulation process have to be investigated in the future [91, 92].

\section{Energy management}

The size of the implant scales directly with the energy consumption as the largest component is the battery. Thus ultra-low power consumption of all components is required in order to minimize the overall size of the implant. An assessment of the energy consumption of the individual components is shown in Table 4.3. The overall energy consumption is a combination of a continuous contribution of all components in standby mode as well as periodically very high contributions by the QCL during a measurement. The standby and operating currents of all components besides the QCL are in the $\mu \mathrm{A}$ range and easy to handle with the supply current of a lithium-ion battery. The high lasing currents of the QCL in the $100 \mathrm{~mA}$ range, however, must be supplied by a capacitor, which is loaded by the battery prior to the event.

The average power consumption of the QCL is assessed as follows. Taking into account the possibility to use only 5 fixed frequency QCLs with a bandwidth of $4 \mathrm{~cm}^{-1}$ in order to gain nearly the same amount of information as with a tuneable QCL, and assuming the same integration time as used for the measurements shown above (leading to the same signal to noise ratio), a time of $5 \mathrm{~s}$ is required for one measurement (1 s per wavenumber). Low power consumption DFB-QCLs as the ones reported e.g. by Katsuyama et al. [95] could therefor be used. These lasers $(7.23 \mu \mathrm{m}$ to $7.27 \mu \mathrm{m}$ wavelength) have a lasing threshold at a current around $52 \mathrm{~mA}$ (using a voltage of $8.4 \mathrm{~V}$, could be supplied by a voltage converter inside the implant) and consume only $0.44 \mathrm{~W}$ at the threshold. In order to achieve sufficient lasing intensities for the application, higher lasing currents are required: A current of $120 \mathrm{~mA}$ results in a conceivable output power of about $5 \mathrm{~mW}$ (at a laser temperature of $40{ }^{\circ} \mathrm{C}$ ) [95]. 12 measurements per hour (one every 5 minutes) are necessary for a sufficient glucose monitoring and thus an overall average power of $18.2 \mathrm{~mW}$ is required just from the laser (assuming $80 \%$ efficiency of the implant's components for current and voltage 
supply).

In total, the power consumption of the device would then be around $18.6 \mathrm{~mW}$. Typical volumetric energy densities of e.g. rechargeable lithium-ion batteries are around $200 \mathrm{Wh} / \mathrm{L}$ to $500 \mathrm{Wh} / \mathrm{L}$ [96]. Thus a battery volume as low as $1.3 \mathrm{~cm}^{3}$ $\left(1.8 \mathrm{~cm}^{3}\right)$ (assuming volumetric energy density of $350 \mathrm{Wh} / \mathrm{L}$ ) is needed for a $24 \mathrm{~h}$ $(33 \mathrm{~h})$ operation time. This means, that the device would have to be recharged every $24 \mathrm{~h}$. This could be done from outside the body via electromagnetic induction (e.g. [97]).

DFB QCLs typically require temperature stabilization by a Peltiér element. This poses obvious issues in an in vivo application. The lasing properties in particular the power and wavelength of the emitted beam depend on the laser's temperature. It is conceivable that the QCL is stabilized by the body temperature which can change by a few degrees during measurements. The low power consumption DFB QCLs reported by Katsuyama et al. [95] change their wavelength by $0.59 \mathrm{~nm} / \mathrm{K}$. In the $10 \mu \mathrm{m}$ wavelength range this results in a wavenumber change of $<0.1 \mathrm{~cm}^{-1} / \mathrm{K}$ and, thus, can be neglected compared to the bandwidth of $4 \mathrm{~cm}^{-1}$. The temperature dependence of lasing intensity in the range of $1 \mathrm{~mW} / \mathrm{K}$ constitutes a larger problem [95]. However, this is not a problem when an additional reference channel is included in the sensor (as proposed earlier).

\section{Data management}

Data handling could be done by a microcontroller integrated into the implant. It manages the measurement process, does the signal filtering and signal referencing, the energy management, data storage as well as the data transmission via a bluetooth module. There are already small, low energy consuming MEMS-based microcontrollers with enough computing power for the application available (e.g. by Microchip Technology Inc., Arizona, USA).

\section{Miniaturization}

An overall size of the implant in the range of few centimeters can be achieved by miniaturization of the individual components. The pyroelectric detectors used in our setups are intrinsically in the mm-range in size. When looking for a small laser, some challenges occur due to the external grating in the EC-QCL as mentioned earlier. Thus the use of fixed-frequency QCLs such as Fabry-Perot or DFB QCLs that are intrinsically small (in the mm-range) seems to be a promising choice to integrate into an implant.

\section{Long-term stability and lifetime}

For a long-term stable system the components itself have to be stable over long periods of time. In Section 3.3 it has been demonstrated, that a stable glucose diffusion into the sensor as well as a stable glucose prediction using one reference measurement for each measurement over a time period of 42 days is possible. As the 


\section{Discussion}

experiment was stopped due to personal capacity reasons, an even longer application of the experiment could have been possible. This makes measurements over periods of several months promising. Due to our proposed sealed referencing pathway external calibration measurements are not required to account for temperature drifts. No problem is seen in the lifetime of the materials, as the optical materials (diamond, stainless steel, silicon) are robust and the electronic materials (microcontroller, battery, laser, detector) are designed for a long lifetime. The most limiting factor will presumably be the lifetime of the rechargeable battery. Typical batteries have a nominal cycle life of $>500$ cycles at $20^{\circ} \mathrm{C}$ (e.g. CP 1454 A3, VARTA Microbattery GMbH, Germany; no details given for higher temperatures), such that a lifetime of $>1$ year could be possible.

\section{Next step towards in vivo}

As an intermediate step prior to full implantation, we suggest the use of the transflection cell as an MIR-optical 'port into the body'. This port could be implanted in the skin whereby the side of the silicon wafer points towards the interstitial fluid and the optical window points towards the outside. This way the optical experimental setup as it is can be flexibly used and modified from outside the body, while the sensor's spectral performance as well as long-term stability and biological response can be investigated in vivo. 


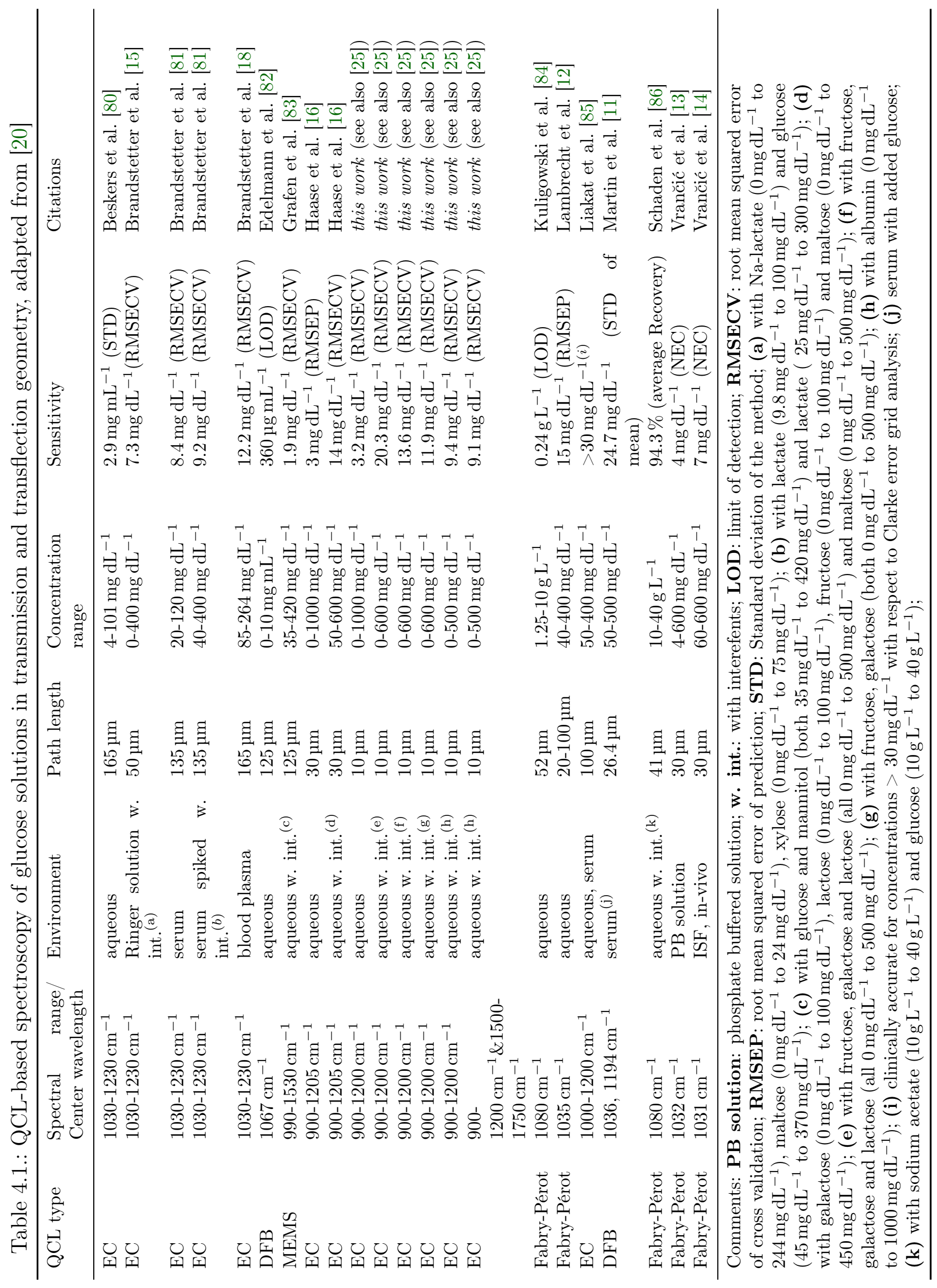




\section{Discussion}

Table 4.2.: Achieved sensitivity (RMSECV) when restricting the amount of wavenumbers. The evaluation of the best wavenumbers was done using an evolutionary algorithm. Table adapted from Isensee et al. [25]

\begin{tabular}{|c|c|c|c|c|c|c|c|c|c|c|c|}
\hline $\begin{array}{l}\# \\
\text { Wavenum- } \\
\text { bers }\end{array}$ & 984 & 1030 & $\begin{array}{l}\text { Wave } \\
108\end{array}$ & $\begin{array}{l}\text { enumb } \\
51099\end{array}$ & $\begin{array}{l}\text { eers } \\
113\end{array}$ & $\begin{array}{c}\left.\mathrm{cm}^{-1}\right] \\
81148\end{array}$ & 1185 & 51195 & 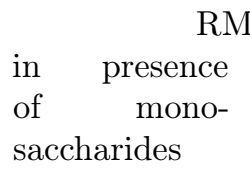 & $\begin{array}{l}\mathrm{SECV}_{\text {cycle }}[\mathrm{mg} / \mathrm{c} \\
\text { in presence of } \\
\text { albumin }\end{array}$ & $\begin{array}{l}\mathrm{dL}] \\
\text { in pure glu- } \\
\text { cose solutions }\end{array}$ \\
\hline 3 & & $\mathrm{X}$ & & & $\mathrm{X}$ & $\mathrm{X}$ & & & 20.4 & 10.2 & 6.5 \\
\hline 5 & & $\mathrm{X}$ & $\mathrm{X}$ & X & & X & $\mathrm{X}$ & & 16.5 & 9.4 & 6.5 \\
\hline 7 & $\mathrm{X}$ & $\mathrm{X}$ & $\mathrm{X}$ & $\mathrm{X}$ & & $\mathrm{X}$ & $\mathrm{X}$ & $\mathrm{X}$ & 15.4 & 9.6 & 6.6 \\
\hline
\end{tabular}

Table 4.3.: Energy consumption of different components included in an implant

\begin{tabular}{|c|c|c|c|c|c|}
\hline Component & $\begin{array}{l}\text { Operation } \\
\text { mode }\end{array}$ & $\begin{array}{l}\text { Average } \\
\text { current } \\
{[\mu \mathrm{A}]}\end{array}$ & Voltage [V] & $\begin{array}{l}\text { Average } \\
\text { Power } \\
{[\mathrm{mW}]}\end{array}$ & Ref. \\
\hline $\begin{array}{l}\text { Bluetooth } \\
\text { module }\end{array}$ & $\begin{array}{l}\text { in standby } \\
\text { receiver active } \\
\text { transmitter } \\
\text { active (1 } \\
\text { frame/second) }\end{array}$ & $\begin{array}{l}2.0 \\
25.0 \\
5.0\end{array}$ & $\begin{array}{l}3.3 \\
3.3 \\
3.3\end{array}$ & $\begin{array}{l}0.01 \\
0.13 \\
0.08\end{array}$ & e.g. [93] \\
\hline $\begin{array}{l}\text { Microcontroller } \\
\text { (with AD- } \\
\text { converter } \\
\text { and lock-in } \\
\text { amplifier) }\end{array}$ & $\begin{array}{lr}2 \% & \text { active } \\
(72 \mathrm{~s} / \mathrm{h}), & 98 \% \\
\text { inactive } & \end{array}$ & 24.0 & 3.3 & 0.08 & \\
\hline $\begin{array}{l}\text { Pyroelectric } \\
\text { detector }\end{array}$ & $\begin{array}{l}2 \% \text { active, } 98 \% \\
\text { inactive }\end{array}$ & 10.8 & 3.0 & 0.04 & e.g. [94] \\
\hline QCL & $\begin{array}{l}2 \% \text { active, } 98 \% \\
\text { inactive }\end{array}$ & & & 18.2 & e.g. [95] \\
\hline
\end{tabular}




\section{Bibliography}

[1] World Health Organization, Global report on diabetes, 2016.

[2] NCD Risk Factor Collaboration (NCD-RisC). Worldwide trends in diabetes since 1980: a pooled analysis of 751 population-based studies with 4.4 million participants. Lancet, 387(10027):1513-1530, Apr 2016. doi: 10.1016/S0140-6736(16)00618-8. URL http://www.ncbi.nlm.nih.gov/pmc/ articles/PMC5081106/.

[3] Till Seuring, Olga Archangelidi, and Marc Suhrcke. The economic costs of type 2 diabetes: A global systematic review. PharmacoEconomics, 33(8):811-831, Aug 2015. doi: 10.1007/s40273-015-0268-9. URL https ://doi.org/10.1007/ s40273-015-0268-9.

[4] International Diabetes Federation, IDF Diabetes Atlas, 2013.

[5] Constantin Ionescu-Tirgoviste, Paul A. Gagniuc, Elvira Gubceac, Liliana Mardare, Irinel Popescu, Simona Dima, and Manuella Militaru. A 3d map of the islet routes throughout the healthy human pancreas. Scientific Reports, 5:14634 EP -, Sep 2015. URL http://dx.doi.org/10.1038/srep14634.

[6] American Diabetes Association. Screening for type 2 diabetes. Diabetes Care, (23 Suppl 1):20-3, 2000.

[7] David Rodbard. Continuous glucose monitoring: A review of recent studies demonstrating improved glycemic outcomes. Diabetes Technol. Ther., 19(Suppl 3):S-25-S-37, Jun 2017. doi: 10.1089/dia.2017.0035[PII]. URL http://www . ncbi.nlm.nih.gov/pmc/articles/PMC5467105/.

[8] David Rodbard. Continuous glucose monitoring: A review of successes, challenges, and opportunities. Diabetes Technol. Ther., 18(Suppl 2):S2-3-S2-13, Feb 2016. doi: 10.1089/dia.2015.0417[PII]. URL http://www.ncbi.nlm.nih. gov/pmc/articles/PMC4717493/.

[9] Raeann Gifford. Continuous glucose monitoring: 40 years, what weve learned and whatś next. ChemPhysChem, 14(10):2032-2044, 2013. doi: 10. 1002/cphc.201300172. URL https://onlinelibrary.wiley.com/doi/abs/ 10.1002/cphc. 201300172. 
[10] Heinz Fabian, Peter Lasch, and Dieter Naumann. Analysis of biofluids in aqueous environment based on mid-infrared spectroscopy. Journal of Biomedical Optics, 10:10-10-10, 2005. doi: 10.1117/1.1917844. URL https://doi.org/ $10.1117 / 1.1917844$

[11] W. Blake Martin, Sergey Mirov, and Ramakrishna Venugopalan. Middle infrared, quantum cascade laser optoelectronic absorption system for monitoring glucose in serum. Applied Spectrosc., 59(7):881-884, Jul 2005. URL http://as.osa.org/abstract. cfm?URI=as $-59-7-881$.

[12] A. Lambrecht, T. Beyer, K. Hebestreit, R. Mischler, and W. Petrich. Continuous glucose monitoring by means of fiber-based, mid-infrared laser spectroscopy. Applied Spectroscopy, 60(7):729-736, 2006. doi: 10.1366/000370206777887071. URL https://doi.org/10.1366/000370206777887071.

[13] C. Vrančić, A. Fomichova, N. Gretz, C. Herrmann, S. Hoecker, A. Pucci, and W. Petrich. Continuous glucose monitoring by means of mid-infrared transmission laser spectroscopy in vitro. Analyst, 136:1192 - 1198, 2011.

[14] Christian Vrančić, Niels Kröger, Norbert Gretz, Sabine Neudecker, Annemarie Pucci, and Wolfgang Petrich. A quantitative look inside the body: Minimally invasive infrared analysis in vivo. Analytical Chemistry, 86(21):10511-10514, 2014. doi: 10.1021/ac5028808. URL https://doi.org/10.1021/ac5028808.

[15] Markus Brandstetter, Andreas Genner, Kresimir Anic, and Bernhard Lendl. Tunable external cavity quantum cascade laser for the simultaneous determination of glucose and lactate in aqueous phase. Analyst, 135:3260-3265, 2010. doi: 10.1039/C0AN00532K. URL http://dx.doi.org/10.1039/COAN00532K.

[16] Katharina Haase, Niklas Müller, and Wolfgang Petrich. Towards a continuous glucose monitoring system using tunable quantum cascade lasers. Proc. SPIE, 10490:10490 - 10490 - 8, 2018. doi: 10.1117/12.2291745. URL https://doi . org/10.1117/12.2291745.

[17] Niklas Müller. Multivariate Glukosebestimmung auf Basis durchstimmbarer Quantenkaskadenlaser, Master thesis. Heidelberg University, Heidelberg, Germany, 2016.

[18] Markus Brandstetter, Tamara Sumalowitsch, Andreas Genner, Andreas E. Posch, Christoph Herwig, Andreas Drolz, Valentin Fuhrmann, Thomas Perkmann, and Bernhard Lendl. Reagent-free monitoring of multiple clinically relevant parameters in human blood plasma using a mid-infrared quantum cascade laser based sensor system. Analyst, 138:4022-4028, 2013. doi: 10.1039/C3AN00300K. URL http://dx.doi.org/10.1039/C3AN00300K.

[19] Mirta R. Alcaráz, Andreas Schwaighofer, Christian Kristament, Georg Ramer, Markus Brandstetter, Héctor Goicoechea, and Bernhard Lendl. External-cavity 
quantum cascade laser spectroscopy for mid-ir transmission measurements of proteins in aqueous solution. Analytical Chemistry, 87(13):6980-6987, 2015. doi: 10.1021/acs.analchem.5b01738. URL https://doi.org/10.1021/acs . analchem.5b01738.

[20] Katharina Isensee, Niels Kröger-Lui, and Wolfgang Petrich. Biomedical applications of mid-infrared quantum cascade lasers - a review. Analyst, Submitted July, 15, 2018.

[21] Anna Fomichova. Bestimmung der Diffusions- und Integrationszeiten für die kontinuierliche Glucosemessung auf Basis der fasergebundenen MittelinfrarotSpektroskopie, Diploma thesis. Heidelberg University, Heidelberg, Germany, 2011.

[22] Christian Vrancîć. Kontinuierliche, reagensfreie Glucosebestimmung, Dissertation. Heidelberg University, Heidelberg, Germany, 2012.

[23] Wolfgang Demtröder. Molekülphysik. Oldenburg Wissenschaftsverlag GmbH, Munich, Germany, 2013.

[24] George M. Hale and Marvin R. Querry. Optical constants of water in the 200-nm to 200- $\mu \mathrm{m}$ wavelength region. Appl. Opt., 12(3):555-563, Mar 1973. doi: 10.1364/AO.12.000555. URL http://ao.osa.org/abstract.cfm?URI= ao-12-3-555.

[25] Katharina Isensee, Niklas Müller, Annemarie Pucci, and Wolfgang Petrich. Towards a quantum cascade laser-based implant for the continuous monitoring of glucose. Analyst, Submitted July, 23, 2018.

[26] Jerome Faist, Federico Capasso, Deborah L. Sivco, Carlo Sirtori, Albert L. Hutchinson, and Alfred Y. Cho. Quantum cascade laser. Science, 264(5158): 553-556, 1994. doi: 10.1126/science.264.5158.553. URL http://science. sciencemag.org/content/264/5158/553.

[27] Andreas Schwaighofer, Markus Brandstetter, and Bernhard Lendl. Quantum cascade lasers (qcls) in biomedical spectroscopy. Chem. Soc. Rev., 46:59035924, 2017. doi: 10.1039/C7CS00403F. URL http://dx.doi.org/10.1039/ C7CS00403F.

[28] Niels Kröger, Alexander Egl, Maria Engel, Norbert Gretz, Katharina Haase, Iris Herpich, Bettina Kränzlin, Sabine Neudecker, Annemarie Pucci, Arthur Schönhals, Jochen Vogt, and Wolfgang Petrich. Quantum cascade laser-based hyperspectral imaging of biological tissue. Journal of Biomedical Optics, 19:19 - 19 - 6, 2014. doi: 10.1117/1.JBO.19.11.111607. URL https://doi .org/10. $1117 / 1$. JBO.19.11.111607. 
[29] Miriam Serena Vitiello, Giacomo Scalari, Benjamin Williams, and Paolo De Natale. Quantum cascade lasers: 20 years of challenges. Opt. Express, 23 (4):5167-5182, Feb 2015. doi: 10.1364/OE.23.005167. URL http://www . opticsexpress .org/abstract. cfm?URI=oe-23-4-5167.

[30] NIST National Institute of Standards and Technology Chemistry. WebBook Ethanol. https: //webbook. nist. gov/chemistry/, Accessed: 2017-12-14.

[31] NIST National Institute of Standards and Technology Chemistry. WebBook Water. https: //webbook. nist. gov/chemistry/, Accessed: 2017-12-14.

[32] M. Born and E. Wolf. Principles of Optics. Pergamon, Oxford, UK, 2nd ed. edition, 1964.

[33] Thomas G. Mayerhöfer and Jürgen Popp. The electric field standing wave effect in infrared transflection spectroscopy. Spectrochim. Acta, Part A, 191:283-289, 2018.

[34] Paul Bassan, Joe Lee, Ashwin Sachdeva, Juliana Pissardini, Konrad M. Dorling, John S. Fletcher, Alex Henderson, and Peter Gardner. The inherent problem of transflection-mode infrared spectroscopic microscopy and the ramifications for biomedical single point and imaging applications. Analyst, 138:144-157, 2013. doi: 10.1039/C2AN36090J. URL http://dx.doi.org/10.1039/C2AN36090J.

[35] Brent M. DeVetter, Seth Kenkel, Shachi Mittal, Rohit Bhargava, and Tomasz P. Wrobel. Characterization of the structure of low-e substrates and consequences for ir transflection measurements. Vib. Spectrosc., 91:119 - 127, 2017. doi: https://doi.org/10.1016/j.vibspec.2016.09.001. URL http://www . sciencedirect.com/science/article/pii/S0924203116302156.

[36] Emilia Staniszewska-Slezak, Anna Rygula, Kamilla Malek, and Malgorzata Baranska. Transmission versus transflection mode in ftir analysis of blood plasma: is the electric field standing wave effect the only reason for observed spectral distortions? Analyst, 140:2412-2421, 2015. doi: 10.1039/ C4AN01842G. URL http://dx.doi.org/10.1039/C4AN01842G.

[37] J. Filik, M. D. Frogley, J. Pijanka, K. Wehbe, and G. Cinque. Optical standing-wave artifacts in reflection-absorption ftir microspectroscopy of biological materials. J. Phys.: Conf. Ser., 359(1):012006, 2012. URL http: //stacks.iop.org/1742-6596/359/i=1/a=012006.

[38] Jacob Filik, Mark D. Frogley, Jacek K. Pijanka, Katia Wehbe, and Gianfelice Cinque. Electric field standing wave artefacts in ftir micro-spectroscopy of biological materials. Analyst, 137:853-861, 2012. doi: 10.1039/C2AN15995C. URL http://dx.doi.org/10.1039/C2AN15995C. 
[39] Tomasz P. Wrobel, Barbara Wajnchold, Hugh J. Byrne, and Malgorzata Baranska. Electric field standing wave effects in $\mathrm{ft}$-ir transflection spectra of biological tissue sections: Simulated models of experimental variability. Vib. Spectrosc., 69:84 - 92, 2013. doi: https://doi.org/10.1016/j.vibspec. 2013.09.008. URL http://www.sciencedirect.com/science/article/pii/ S0924203113001161.

[40] Heather Brooke, B. V. Bronk, J. N. McCutcheon, S. L. Morgan, and M. L. Myrick. A study of electric field standing waves on reflection microspectroscopy of polystyrene particles. Appl. Spectrosc., 63(11):1293-1302, 2009. doi: 10.1366/ 000370209789806902 . URL https://doi .org/10.1366/000370209789806902.

[41] R. M. A. Azzam and N. M. Bashara. Ellipsometry and Polarized Light. Elsevier Science B.V., Amsterdam, The Netherlands, paperback ed. edition, 1987.

[42] David F. Edwards and Ellen Ochoa. Infrared refractive index of diamond. J. Opt. Soc. Am., 71(5):607-608, May 1981. doi: 10.1364/JOSA.71.000607. URL http://www . osapublishing.org/abstract.cfm?URI=josa-71-5-607.

[43] T. P. Mollart and K. L. Lewis. The infrared optical properties of cvd diamond at elevated temperatures. physica status solidi (a), 186(2):309-318, 2001. doi: 10.1002/1521-396X(200108)186:2<309::AID-PSSA309>3.0.CO;2-I. URL https://onlinelibrary.wiley.com/doi/abs/10.1002/1521-396X\% $28200108 \% 29186 \% 3$ A2\%3C309\%3A\%3AAID-PSSA309\%3E3. 0. CO \%3B2-I.

[44] John E. Bertie and Zhida Lan. Infrared intensities of liquids xx: The intensity of the oh stretching band of liquid water revisited, and the best current values of the optical constants of h2o(l) at 25c between 15,000 and $1 \mathrm{~cm}-1$. Applied Spectroscopy, 50(8):1047-1057, 1996. doi: 10.1366/0003702963905385. URL https://doi.org/10.1366/0003702963905385.

[45] Jens Trollmann and Annemarie Pucci. Infrared dielectric function of gold films in relation to their morphology. The Journal of Physical Chemistry C, 118 (27):15011-15018, 2014. doi: 10.1021/jp5027465. URL https://doi.org/10. 1021/jp5027465.

[46] William D. Jr. Callister. Materials Science and Engineering: An Introduction. John Wiley \& Sons, Inc, New Jersey, USA, 2007.

[47] Glen A. Slack and S. F. Bartram. Thermal expansion of some diamondlike crystals. Journal of Applied Physics, 46(1):89-98, 1975. doi: 10.1063/1.321373. URL https://doi .org/10.1063/1.321373.

[48] Yasumasa Okada and Yozo Tokumaru. Precise determination of lattice parameter and thermal expansion coefficient of silicon between 300 and $1500 \mathrm{k}$. Journal of Applied Physics, 56(2):314-320, 1984. doi: 10.1063/1.333965. URL https://doi.org/10.1063/1.333965. 
[49] David J. Segelstein. The complex refractive index of water, Master thesis. University of Missouri-Kansas City, Kansas City, Missouri, USA, 1981.

[50] John E. Bertie, M. Khalique Ahmed, and Hans H. Eysel. Infrared intensities of liquids. 5. optical and dielectric constants, integrated intensities, and dipole moment derivatives of water and water-d2 at 22.degree.c. The Journal of Physical Chemistry, 93(6):2210-2218, 1989. doi: 10.1021/j100343a008. URL https://doi.org/10.1021/j100343a008.

[51] Harry D. Dowing and Dudley Williams. Optical constants of water in the infrared. Journal of Geophysical Research, 80(12):1656-1661, April 1975. doi: 10.1029/JC080i012p01656.

[52] Lary W. Pinkley, P. P. Sethna, and Dudley Williams. Optical constants of water in the infrared: Influence of temperature*. J. Opt. Soc. Am., 67(4):494-499, Apr 1977. doi: 10.1364/JOSA.67.000494. URL http://www.osapublishing. org/abstract. cfm?URI=josa-67-4-494.

[53] John Bertie's Download Site. https: // sites. ualberta. ca/ ¡jbertie/ JBDownload. HTM, Accessed: 2018-05-08.

[54] V. M. Zolotarev, B. A. Mikhailov, L. I. Alperovich, and S. I. Popov. Dispersion and Absorption of Liquid Water in the Infrared and Radio Regions of the Spectrum. Optics and Spectroscopy, 27:430, November 1969.

[55] Charles W. Robertson and Dudley Williams. Lambert absorption coefficients of water in the infrared*. J. Opt. Soc. Am., 61(10):1316-1320, Oct 1971. doi: 10.1364/JOSA.61.001316. URL http://www.osapublishing.org/abstract . cfm?URI=josa-61-10-1316.

[56] David M. Wieliczka, Shengshan Weng, and Marvin R. Querry. Wedge shaped cell for highly absorbent liquids: infrared optical constants of water. Appl. Opt., 28(9):1714-1719, May 1989. doi: 10.1364/AO.28.001714. URL http: //ao.osa.org/abstract. cfm?URI=ao-28-9-1714.

[57] Peter Snoer Jensen, Jimmy Bak, and Stefan Andersson-Engels. Influence of temperature on water and aqueous glucose absorption spectra in the nearand mid-infrared regions at physiologically relevant temperatures. Applied Spectroscopy, 57(1):28-36, 2003. doi: 10.1366/000370203321165179. URL https://doi.org/10.1366/000370203321165179.

[58] Matej Praprotnik, Dušanka Janežič, and Janez Mavri. Temperature dependence of water vibrational spectrum: A molecular dynamics simulation study. The Journal of Physical Chemistry A, 108(50):11056-11062, 2004. doi: 10.1021/ jp046158d. URL https://doi.org/10.1021/jp046158d. 
[59] Y. Maréchal. Infrared spectra of water. i. effect of temperature and of $\mathrm{h} / \mathrm{d}$ isotopic dilution. The Journal of Chemical Physics, 95(8):5565-5573, 1991. doi: 10.1063/1.461630. URL https://doi.org/10.1063/1.461630.

[60] Y. Maréchal. Ir spectroscopy of an exceptional h-bonded liquid: water. Journal of Molecular Structure, 322:105 - 111, 1994 . doi: https://doi. org/10.1016/0022-2860(94)87025-X. URL http://www. sciencedirect.com/ science/article/pii/002228609487025X.

[61] M. E. Thomas and W. J. Tropf. Optical properties of diamond. Johns Hopkins APL Technical Digest, 14:16-23, March 1993.

[62] H. R. Phillip and E. A. Taft. Kramers-kronig analysis of reflectance data for diamond. Phys. Rev., 136:A1445-A1448, Nov 1964. doi: 10.1103/PhysRev.136. A1445. URL https://link.aps.org/doi/10.1103/PhysRev.136.A1445.

[63] Thomas Skov, Frans van den Berg, Giorgio Tomasi, and Rasmus Bro. Automated alignment of chromatographic data. Journal of Chemometrics, 20(1112):484-497, 2006. doi: 10.1002/cem.1031. URL https://onlinelibrary . wiley.com/doi/abs/10.1002/cem.1031.

[64] S.D. Brown and R.T. Ferré. Comprehensive Chemometrics: Applications, cumulative index. Comprehensive Chemometrics: Chemical and Biochemical Data Analysis. Elsevier, 2009. ISBN 9780444527066.

[65] Giorgio Tomasi, Frans van den Berg, and Claus Andersson. Correlation optimized warping and dynamic time warping as preprocessing methods for chromatographic data. Journal of Chemometrics, 18(5):231-241, 2004. doi: 10. 1002/cem.859. URL https://onlinelibrary.wiley.com/doi/abs/10.1002/ cem. 859 .

[66] Kenneth R. Beebe and Bruce R. Kowalski. An introduction to multivariate calibration and analysis. Analytical Chemistry, 59(17):1007A-1017A, 1987. doi: 10.1021/ac00144a001. URL https://doi .org/10.1021/ac00144a001.

[67] Harald Martens and Tormod Naes. Multivariate Calibration. John Wiley \& Sons Ltd., 1989. ISBN 978-0471930471.

[68] J. L. Parkes, S. L. Slatin, S. Pardo, and B. H. Ginsberg. A new consensus error grid to evaluate the clinical significance of inaccuracies in the measurement of blood glucose. Diabetes Care, 23(8):1143-1148, 2000. doi: 10.2337/diacare.23. 8.1143. URL http://care.diabetesjournals.org/content/23/8/1143.

[69] Gerold Adam, Peter Läuger, and Günther Stark. Physikalische Chemie und Biophysik. Springer-Verlag Berlin Heidelberg, Amsterdam, The Netherlands, 5 edition, 2009. 
[70] J. Crank. The mathematics of diffusion / by J. Crank. Clarendon Press Oxford [England], 2nd ed. edition, 1975. ISBN 01985334460198534116.

[71] R. L. Fournier. Basic Transport Phenomena in Biomedical Engineering, 2nd Edition. Taylor \& Francis, 2006. ISBN 9781591690269. URL https://books . google.de/books?id=cF7DQgAACAAJ.

[72] M. A. Cox, K. O. Lewis, and B. T. Cooper. Sucrosemia in untreated celiac disease (a potential screening test). Dig. Dis. Sci., 43(5):1096-1101, May 1998. doi: 10.1023/A:1018851306328. URL https://doi.org/10.1023/A : 1018851306328.

[73] Laurie A. Drozdowski and Alan B. R. Thomson. Intestinal sugar transport. World J Gastroenterol., 12(11):1657-1670, 2006.

[74] Hilke Jungen, Hilke Andresen-Streichert, Alexander Müller, and Stefanie Iwersen-Bergmann. Disaccharides in urine samples as markers of intravenous abuse of methadone and buprenorphine. J. Anal. Toxicol., 37(9):652-658, 2013. doi: 10.1093/jat/bkt086. URL http://dx.doi.org/10.1093/jat/bkt086.

[75] N. Fogh-Andersen, B. M. Altura, B. T. Altura, and O. Siggaard-Andersen. Composition of interstitial fluid. Clinical Chemistry, 41(10):1522-1525, 1995. URL http://clinchem.aaccjnls.org/content/41/10/1522.

[76] Martin Ellmerer, Lukas Schaupp, Gernot A. Brunner, Gerald Sendlhofer, Andrea Wutte, Paul Wach, and Thomas R. Pieber. Measurement of interstitial albumin in human skeletal muscle and adipose tissue by open-flow microperfusion. Am. J. Physiol., 278(2):E352-E356, 2000. doi: 10.1152/ajpendo.2000. 278.2.E352. URL https://doi.org/10.1152/ajpendo.2000.278.2.E352.

[77] Katharina Haase. Zentrale Aspekte der Detektion für die Quantenkaskadenlaser-basierte Infrarotmikroskopie, Bachelor thesis. Heidelberg University, Heidelberg, Germany, 2012.

[78] Eugene M. Renkin. Filtration, diffusion, and molecular sieving through porous cellulose membranes. The Journal of General Physiology, 38(2):225-243, 1954. doi: 10.1085/jgp.38.2.225. URL http://jgp.rupress.org/content/38/2/ 225.

[79] Robert E. Beck and Jerome S. Schultz. Hindrance of solute diffusion within membranes as measured with microporous membranes of known pore geometry. Biochimica et Biophysica Acta (BBA) - Biomembranes, 255(1):273 303, 1972. doi: https://doi.org/10.1016/0005-2736(72)90028-4. URL http: //www.sciencedirect.com/science/article/pii/0005273672900284. 
[80] Timo F. Beskers, Markus Brandstetter, Julia Kuligowski, Guillermo Quintás, Manfred Wilhelm, and Bernhard Lendl. High performance liquid chromatography with mid-infrared detection based on a broadly tunable quantum cascade laser. Analyst, 139:2057-2064, 2014. doi: 10.1039/C3AN01546G. URL http://dx.doi.org/10.1039/C3AN01546G.

[81] M. Brandstetter, L. Volgger, A. Genner, C. Jungbauer, and B. Lendl. Direct determination of glucose, lactate and triglycerides in blood serum by a tunable quantum cascade laser-based mid-ir sensor. Applied Physics B, 110(2):233-239, Feb 2013. doi: 10.1007/s00340-012-5080-z. URL https://doi.org/10.1007/ s00340-012-5080-z.

[82] Andrea Edelmann, Caterina Ruzicka, Johannes Frank, Bernhard Lendl, Werner Schrenk, Erich Gornik, and Gottfried Strasser. Towards functional groupspecific detection in high-performance liquid chromatography using midinfrared quantum cascade lasers. Journal of Chromatography A, 934(1):123 - 128, 2001. doi: https://doi.org/10.1016/S0021-9673(01)01288-2. URL http: //www.sciencedirect.com/science/article/pii/S0021967301012882.

[83] M. Grafen, S. Delbeck, H. Busch, H. M. Heise, and A. Ostendorf. Evaluation and benchmarking of an ec-qcl-based mid-infrared spectrometer for monitoring metabolic blood parameters in critical care units. Proc.SPIE, 10501:10501 10501 - 10, 2018. doi: 10.1117/12.2289625. URL https://doi.org/10.1117/ 12. 2289625 .

[84] J. Kuligowski, G. Quintás, and B. Lendl. High performance liquid chromatography with on-line dual quantum cascade laser detection for the determination of carbohydrates, alcohols and organic acids in wine and grape juice. Applied Physics B, 99(4):833-840, Jun 2010. doi: 10.1007/s00340-010-4013-y. URL https://doi.org/10.1007/s00340-010-4013-y.

[85] Sabbir Liakat, Kevin A. Bors, Tzu-Yung Huang, Anna P. M. Michel, Eric Zanghi, and Claire F. Gmachl. In vitro measurements of physiological glucose concentrations in biological fluids using mid-infrared light. Biomedical Optics Express, 4(7):1083-1090, Jul 2013. doi: 10.1364/BOE.4.001083. URL http://www . osapublishing. org/boe/abstract. cfm?URI=boe-4-7-1083.

[86] S. Schaden, A. Domínguez-Vidal, and B. Lendl. Simultaneous measurement of two compounds in aqueous solution with dual quantum cascade laser absorption spectroscopy. Applied Physics B, 83(1):135-139, Apr 2006. doi: 10.1007/ s00340-006-2133-1. URL https://doi .org/10.1007/s00340-006-2133-1.

[87] B. G. Lee, M. A. Belkin, C. Pflugl, L. Diehl, H. A. Zhang, R. M. Audet, J. MacArthur, D. P. Bour, S. W. Corzine, G. E. Hofler, and F. Capasso. Dfb quantum cascade laser arrays. IEEE Journal of Quantum Electronics, 45(5): 554-565, May 2009. ISSN 0018-9197. doi: 10.1109/JQE.2009.2013175. 
[88] Marcia W. Patchan, J. Jeremy Chae, Justin D. Lee, Xiomara Calderon-Colon, Jeffrey P. Maranchi, Russell L. McCally, Oliver D. Schein, Jennifer H. Elisseeff, and Morgana M. Trexler. Evaluation of the biocompatibility of regenerated cellulose hydrogels with high strength and transparency for ocular applications. Journal of Biomaterials Applications, 30(7):1049-1059, 2016. doi: 10.1177/ 0885328215616273 . URL https://doi .org/10.1177/0885328215616273.

[89] Takeaki Miyamoto, Shin-ichi Takahashi, Hiraku Ito, Hiroshi Inagaki, and Yasuhara Noishiki. Tissue biocompatibility of cellulose and its derivatives. Journal of Biomedical Materials Research, 23(1):125-133, 1989. doi: 10. 1002/jbm.820230110. URL https://onlinelibrary.wiley.com/doi/abs/ $10.1002 / \mathrm{jbm} .820230110$.

[90] Stepan Dobrodey. Passivierung von Mittelinfrarot-Lichtleitfasern durch Polyethylen-Beschichtung für die kontinuierliche, reagensfreie Glucosebestimmung, Bachelor thesis. Heidelberg University, Heidelberg, Germany, 2012.

[91] James M. Anderson, Analiz Rodriguez, and David T. Chang. Foreign body reaction to biomaterials. Seminars in Immunology, 20(2):86 - 100, 2008. doi: https://doi.org/10.1016/j.smim.2007.11.004. URL http://www . sciencedirect.com/science/article/pii/S1044532307000966.

[92] Mahender Nath Avula, Archana Nagaraja Rao, Lawrence D. McGill, David William Grainger, and Florian Solzbacher. Modulation of the foreign body response to implanted sensor models through device-based delivery of the tyrosine kinase inhibitor, masitinib. Biomaterials, 34(38):9737 - 9746, 2013. doi: https://doi.org/10.1016/j.biomaterials.2013.08.090. URL http: //www.sciencedirect.com/science/article/pii/S0142961213010685.

[93] nRF8001, Nordic Semiconductor, Trondheim, Norway, http://www.nordicsemi.com/eng/Products/Bluetooth-low-energy/nRF8001, Accessed: 2018-06-19.

[94] LME336, Infratec GmbH, Dresden, Germany, http://www.infratec.de/fileadmin/media/Sensorik/pdf/LME-336.pdf, Accessed: 2018-06-19.

[95] Tsukuru Katsuyama, Jun ichi Hashimoto, Hiroyuki Yoshinaga, Hiroki Mori, Yukihiro Tsuji, Makoto Murata, Mitsuru Ekawa, and Toshiyuki Tanahashi. Low power-consumption quantum cascade lasers. Proc. SPIE, 9370:9370 - 9370 - 6, 2015. doi: 10.1117/12.2077523. URL https://doi.org/10.1117/12.2077523.

[96] P. Kurzweil and O.K. Dietlmeier. Elektrochemische Speicher: Superkondensatoren, Batterien, Elektrolyse-Wasserstoff, Rechtliche Rahmenbedingungen. Springer Fachmedien Wiesbaden, 2018. ISBN 9783658218294. URL https://books .google.de/books?id=Ml1oDwAAQBAJ. 
[97] Bert Lenaerts and Robert Puers. An inductive power link for a wireless endoscope. Biosensors and Bioelectronics, 22(7):1390 - 1395, 2007. doi: https: //doi.org/10.1016/j.bios.2006.06.015. URL http://www.sciencedirect.com/ science/article/pii/S0956566306002892.

\section{Publications of the author}

This thesis is partly based on the following publications:

- Towards a quantum cascade laser-based implant for the continuous monitoring of glucose

Katharina Isensee, Niklas Müller, Annemarie Pucci, and Wolfgang Petrich. Analyst, Submitted: July, 23, 2018.

- Biomedical applications of mid-infrared quantum cascade lasers - a review

Katharina Isensee, Niels Kröger-Lui, and Wolfgang Petrich.

Analyst, Submitted: July, 15, 2018.

- Towards a continuous glucose monitoring system using tunable quantum cascade lasers

Katharina Haase, Niklas Müller, and Wolfgang Petrich.

Proc. SPIE, 10490:10490 - 10490 - 8, 2018.

Furthermore, the author has contributed to the following publications:

- Real-time quantum cascade laser-based infrared microspectroscopy in-vivo

N. Kröger-Lui*, K. Haase*, A. Pucci, A. Schönhals, and W. Petrich. * These authors contributed equally to this work

Proc. SPIE, 9704, 97040J-97040J-8, 2016.

- Real-time mid-infrared imaging of living microorganisms

Katharina Haase, Niels Kröger-Lui, Annemarie Pucci, Arthur Schönhals, and Wolfgang Petrich.

Journal of Biophotonics, 9, 61-66, 2016.

- Advancements in quantum cascade laser-based infrared microscopy of aqueous media

Katharina Haase*, Niels Kroger-Lui*, Annemarie Pucci, Arthur Schonhals, and Wolfgang Petrich. * These authors contributed equally to this work Faraday Discuss., 187, 119-134, 2016.

- Rapid identification of goblet cells in unstained colon thin sections by means of quantum cascade laser-based infrared microspectroscopy Niels Kröger-Lui, Norbert Gretz, Katharina Haase, Bettina Kränzlin, Sabine Neudecker, Annemarie Pucci, Alexander Regenscheit, Arthur Schönhals, and Wolfgang Petrich.

Analyst, 140, 2086-2092, 2015. 
- Quantum cascade laser-based hyperspectral imaging of biological tissue

Niels Kröger, Alexander Egl, Maria Engel, Norbert Gretz, Katharina Haase, Iris Herpich, Bettina Kränzlin, Sabine Neudecker, Annemarie Pucci, Arthur Schönhals, Jochen Vogt, and Wolfgang Petrich.

Journal of Biomedical Optics, 19(11), 111607, 2014.

- Rapid hyperspectral imaging in the mid-infrared

N. Kröger, A. Egl, M. Engel, N. Gretz, K. Haase, I. Herpich, S. Neudecker, A. Pucci, A. Schönhals, and W. Petrich.

Proc. SPIE, 8939, 89390Z-7, 2014. 


\section{A. Appendix}

\section{A.1. Investigation of the impact of temperature dependence of the individual components on the overall measured transflectance}

In order to visualize the individual contributions of a temperature change on the transflectance, the individual parameters (water layer thickness $d_{2}$, real refractive index $n_{2}$ and absorptivity of water $\alpha_{2}$ ) are varied individually and the overall transflectance signal is calculated as described in Section 2.1.4. This is done by varying one parameter between at its values corresponding to $20^{\circ} \mathrm{C}$ to $40^{\circ} \mathrm{C}$ and keeping all other parameters fixed at their value corresponding to $30^{\circ} \mathrm{C}$. The resulting spectral temperature dependence (calculated by a linear fit on absorbance vs. temperature at every single wavenumber) is shown in Fig. A.1 and its average, maximum and minimum values are summarized in Table A.1.

Table A.1.: Overview on the order of magnitude of the temperature dependence of the (decadic) transflectance with respect to the individual varied temperature dependent contribution

\begin{tabular}{lrrr} 
& \multicolumn{2}{c}{ Temperature dependence $\left[1 /{ }^{\circ} \mathrm{C}\right]$} & Mean \\
\hline$n_{2}$ & Minimum & Maximum & -0.0047 \\
$\alpha_{2}$ & -0.0002 & 0.0045 & -0.0038 \\
$d_{2}$ at $20^{\circ} \mathrm{C}$ & -0.0008 & 0.0011 & -0.0283 \\
$d_{2}$ at $40^{\circ} \mathrm{C}$ & -0.0043 & 0.0194 & 0.0194
\end{tabular}




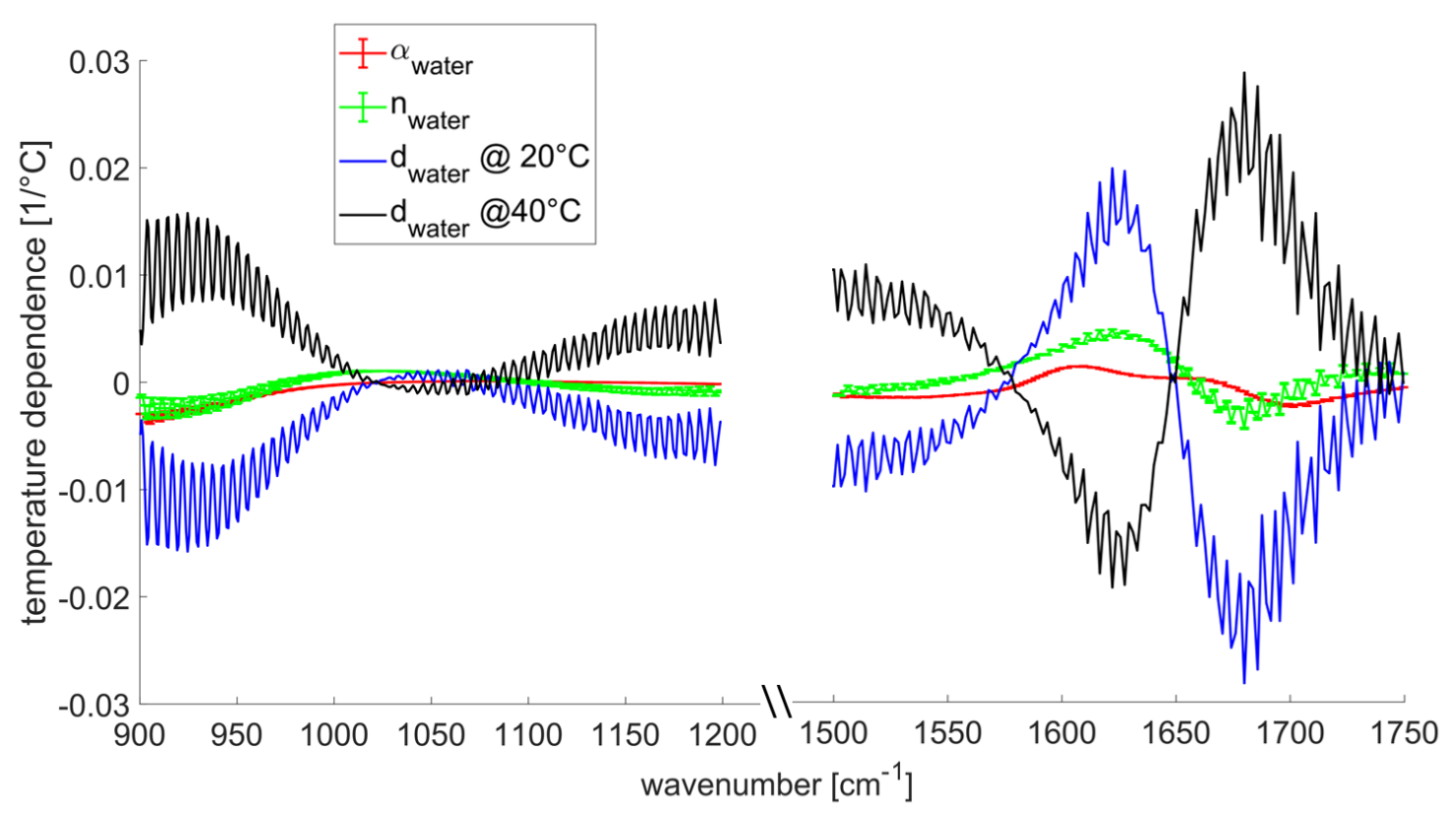

Figure A.1.: Simulation of the change in (decadic) transflectance with temperature with all other components held fixed at their value at $30^{\circ} \mathrm{C}$, respectively. The legend indicates the parameter which was varied. The water layer thickness was chosen to be $d_{2}=10 \mu \mathrm{m}$.

\section{A.2. Simulated absorbance spectra of hypothetical absorber}

Using a hypothetical absorber with a Lorentz-shaped absorptivity the resulting transflectance is calculated according to Section 2.1.4. Figure A.2 gives an overview on the simulated transflectance in the spectral region of the two lasers with respect to the water layer thickness $d_{2}$. Figure A.3 shows the maximum of transflectance with respect to the water layer thickness $d_{2}$. As expected, the dependence of the maximum transflectance to the water layer thickness is not linear (as expected from transmission) but periodically modulated due to interference effects of the transflection geometry. 

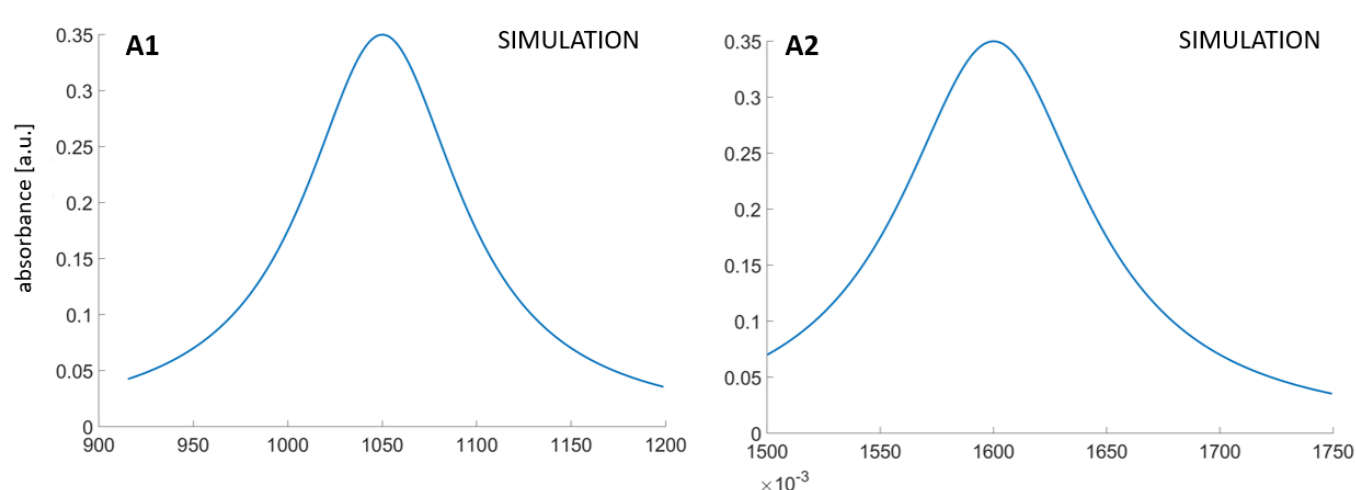

$\mathrm{d}_{2}=15 \mu \mathrm{m}$
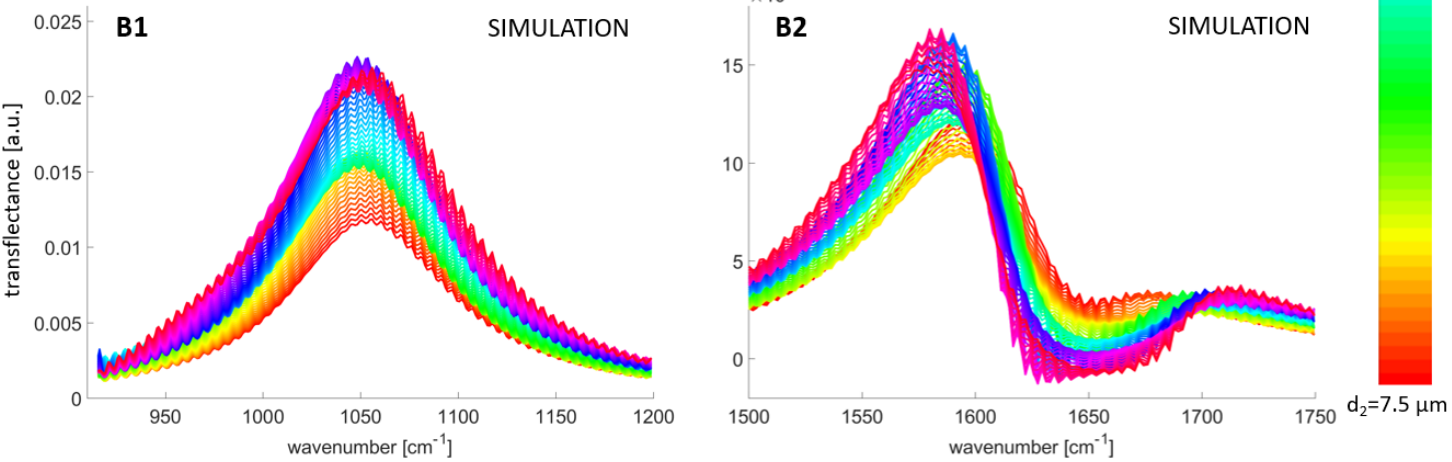

Figure A.2.: A1 and A2: Profile of hypothetical absorber (Lorentz shape with a width of $100 \mathrm{~cm}^{-1}$ ) as it is e.g. measured in transmission; B1 and B2 corresponding transflectance spectra simulated for a transflection geometry with various layer thicknesses between $7.5 \mu \mathrm{m}$ to $15 \mu \mathrm{m}$ visualized by color. 

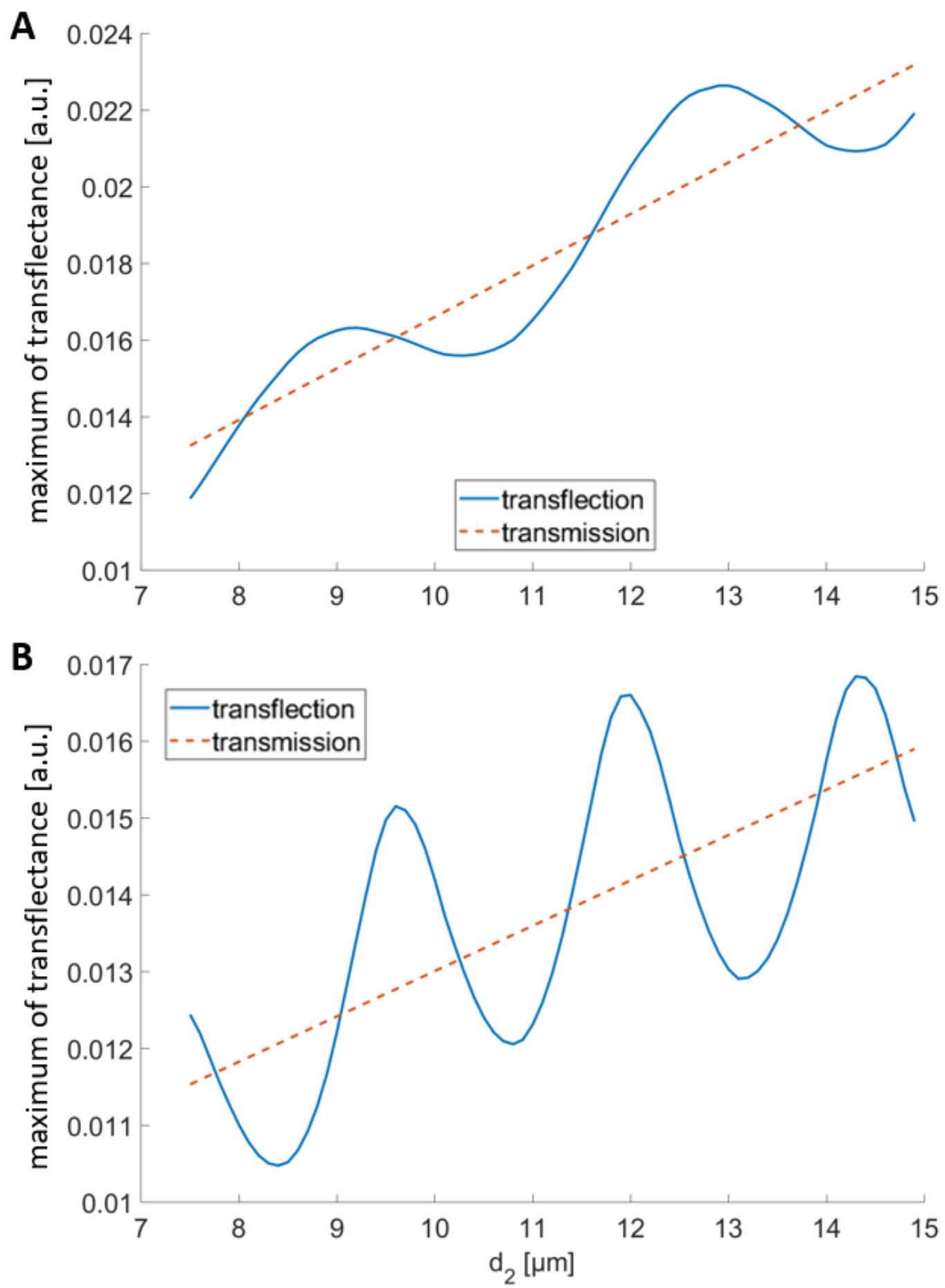

Figure A.3.: Maximum value of simulated transflectance as shown in Figure A.2 with respect to layer thickness $d_{2}$ for QCLG $(\mathbf{A})$ and QCLP $(\mathbf{B})$. The curve shows a periodical modulation with layer thickness in contrast to a linear dependence at it is expected from a transmission setup. 


\section{A.3. Temperature progress curves}

In the following, the reason for the difference in temperature between the individual temperature ramps for glucose (see Section 3.1.2 Figure 3.15) is described. This difference is caused by a deviation of the equilibrium starting point which can be seen in the temperature progress shown in Fig. A.4): the starting temperatures of every ramp (i.e. the highest temperature) is different between the individual glucose ramps whereas it is approximately the same for all water ramps. This leads to an absolute shift between readout and real sensor temperature which itself is dependent on the starting temperature. 
A. Appendix

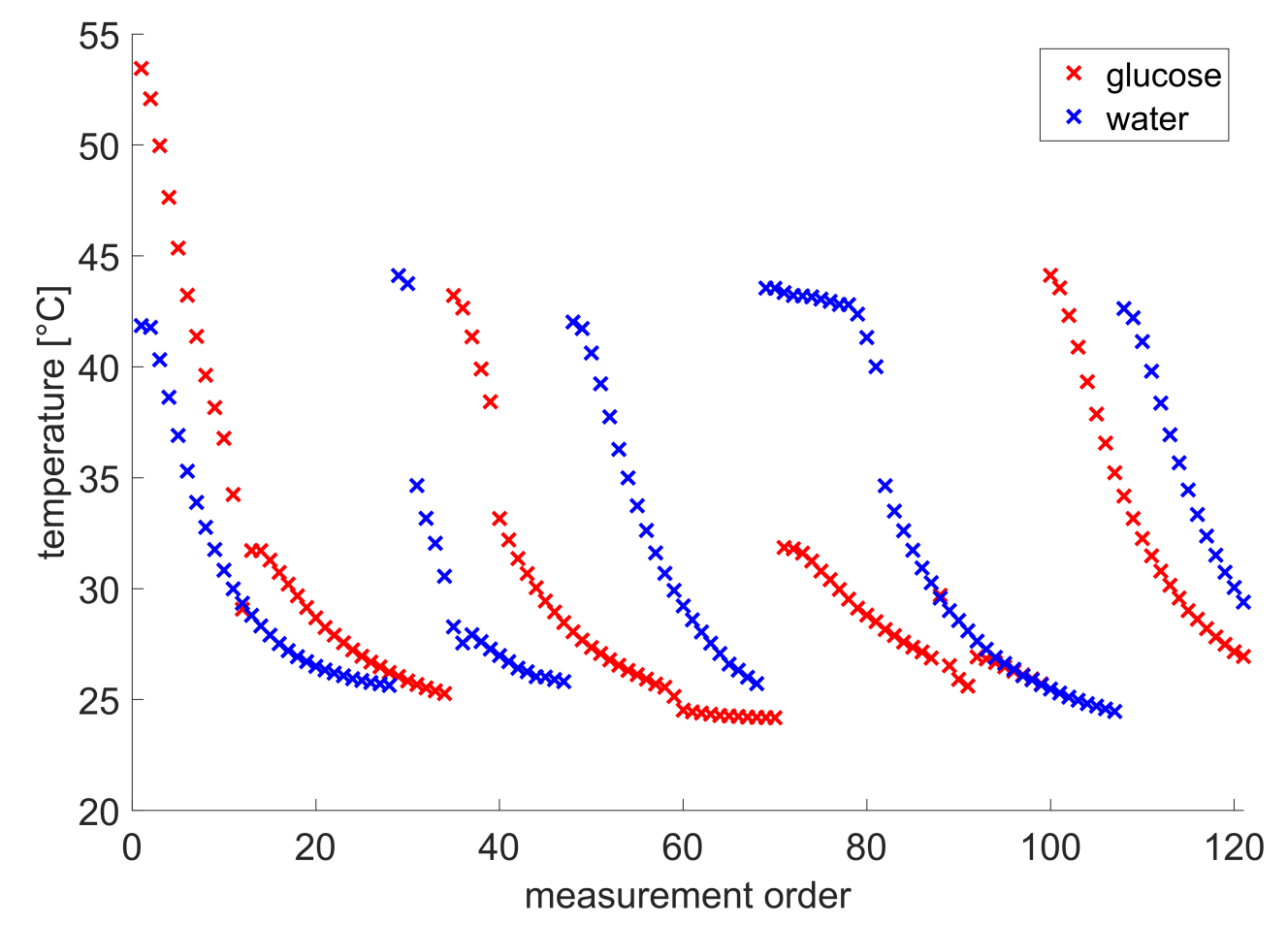

Figure A.4.: Temperature progress with respect to the measurement order of the measurements demonstrated in Sec. 3.1.2. The red crosses indicate glucose measurements whereas the blue ones indicate water measurements. While the maximum temperatures of every ramp are in the same range for water, they are chosen differently for glucose measurements. 




\section{Danksagung}

Zuallererst möchte ich meinem Doktorvater Prof. Dr. Wolfgang Petrich danken. Dafür, dass er mir die Chance gegeben hat meine Promotion in seiner Arbeitsgruppe durchzuführen. Seine wissenschaftlichen und persönlichen Beiträge haben mir sehr geholfen und ich danke ihm auch für das Vertrauen und die motivierende Art mit der er mir entgegengekommen ist. Ich danke für die Möglichkeiten meine Ideen und Motivation am Projekt ausleben zu können. Ich bin ihm außerdem sehr dankbar dafür, dass er sich auch bei Roche immer für mich und mein Projekt eingesetzt hat und sich mir dadurch sehr viele Möglichkeiten über den Tellerrand zu schauen ergeben haben.

Ich danke Frau Prof. Dr. Pucci für die Betreuung, die gute Zusammenarbeit und die anregenden Diskussionen und Anmerkungen zu den Inhalten meiner Forschung.

Ich danke Herrn Prof. Dr. Bachert für die Übernahme der Zweitbegutachtung dieser Arbeit.

Ich danke der HGSFP für die finanzielle Unterstützung zum Beispiel für Konferenzreisen und das Übergangs-Stipendium, welches es mir ermöglichte meine Doktorarbeit fertigzustellen.

Ich danke der ganzen Biophotonik Arbeitsgruppe. Besonders Niklas Müller für die tolle Zusammenarbeit während seiner Masterarbeitszeit und dafür, dass er gewinnbringende Beiträge zu den Vorexperimenten meiner Dissertation gemacht hat. Ein weiterer besonderer Dank gilt Niels Kröger-Lui für seine motivierende Art und die Ideen, Tipps und persönlichen Gespräche. Außerdem danke ich Arthur Schönhals, Jan Spichtinger und Carlos Brandl für die Zeit und nette Büroatmosphäre am KIP.

Ich danke den Kollegen aus der Arbeitsgruppe von Prof. Pucci, insbesondere Christian Huck und Michael Tzschoppe für die Diskussionen und Hilfe bei Fragestellungen in ihrem Spezialgebiet und natürlich für die nette Zeit in den Pausen am KIP.

Ein Dankeschön geht auch an alle Kollegen bei Roche. Insbesondere möchte ich Frank Deck, Christoph Pape und Astrid Wetzler danken für die nette Aufnahme im Büro und die konstruktive Hilfe bei Fragestellungen aller Art. Ein großer Dank geht auch an Dr. Julien Boef für seine Hilfe mit den Fluidiksimulationen und die netten Diskussionen.

Ich danke allen Mitarbeitern der KIP Werkstatt und Elektronik insbesondere Herrn Spiegel und Herrn Azerot für die Hilfe und Tipps während meiner Zeit am KIP.

Ich danke von Herzen meiner Familie für die vielseitige Unterstützung während meiner Doktorarbeit und auch während meines gesamten Studiums, insbesondere meinen Eltern, die immer an mich geglaubt haben.

$\mathrm{Zu}$ guter Letzt möchte ich mich bei meinem Mann Fabian bedanken, dafür dass er mich während der Arbeit und auch sonst immer unterstützt und immer an mich glaubt. Danke! 



\section{B. Erklärung}

Ich versichere, dass ich diese Arbeit selbstständig verfasst habe und keine anderen als die angegebenen Quellen und Hilfsmittel benutzt habe.

Heidelberg, den 1. Oktober 2018 